\title{
General Theory of Intelligence
}

Preconditions for Emergence, General Principles of Functioning and Construction of Nervous System of Living Organisms 


\section{Table of Contents}

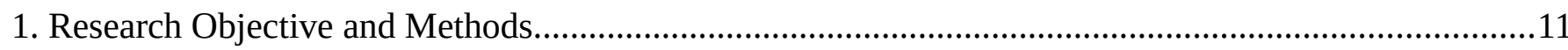

2. Basic Assumptions Accepted during Construction..........................................................................11

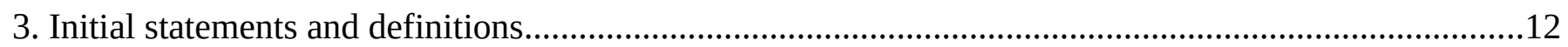

3.1. Properties of Environment (Universe) in which Intelligent Life Develops...................................12

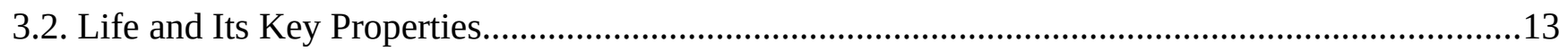

4. Key Functions, Principles of Interaction and Simplified Schematic Diagram of Nervous System.........15

4.1. Basics of Functioning and Approaches to Construction of Nervous System of Living Organism.....

4.1.1. Living Organism as Holistic System of Subsystems Interacting in Coordinated and Targeted Manner.

4.1.2. Enhancement of Efficiency and Effectiveness in Achieving TEFs through Change of Properties of Living Organism's Processes......................................................................16

4.1.3. List of Key Functions of Nervous System of a Living Organism.......................................17

4.2. Interaction of Nervous System with External Processes..............................................................18

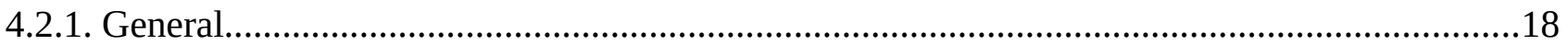

4.2.2. Perception of External Processes as Corresponding to Them Sequence of Signal Combinations - Changes of Characteristics of Certain Processes of Nervous System........19

4.2.3. Influence on External Processes by Generating Sequences of Signal Combinations Changes of Characteristics of Certain (Those that can Influence External Processes) Processes of Nervous System.

4.3. Minimal Block Diagram of Nervous System Structure.............................................................20

5. General Structure, Properties, and Principles of Construction of Nervous System...............................23

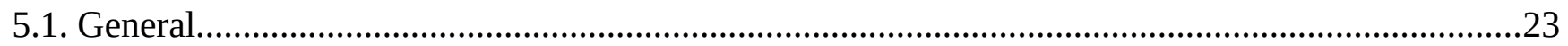

5.2. Predetermination of Properties of Nervous System during its Formation.....................................23

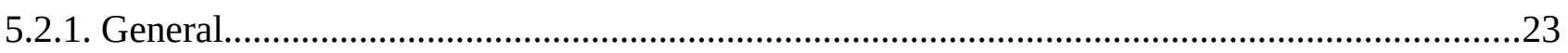

5.2.2. Dependence of Initial Set of Processes of Nervous System of Organism, Their Properties, Values of Characteristics, Structure of Interrelation and Interrelationships on Strategy of Achieving TEFs of Species to which this Organism Belongs.............................24

5.2.3. Preliminary Determination of Properties of Nervous System's Processes that Determine Rules of Change of These Processes.....................................................................................24

5.2.4. Predetermination of Blocks of Preprogrammed Reactions....................................................25

5.2.5. Predetermination of Detectors of State and Probability of TEF Achievement........................25

5.2.6. Predetermined Determination of Adjustment of Self-adjusting Processes of Nervous System to Interaction with Processes of the Universe under Influence of Signals from Blocks of Analysis of State and Probability of TEF Achievement and Preprogrammed

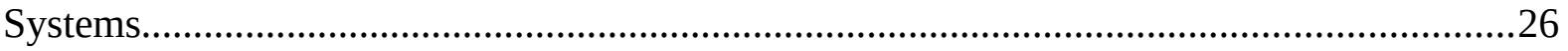

5.2.7. Substrategy of Strategy of Achieving TEFs of Organism...................................................26 
5.3. Limitation of Resources - Limitation of Capabilities and Optimization of Nervous System .27

5.3.1. Limitation of Sets of Detected and Generated Signals as a Result of Limitation of Resources.

5.3.2. Limitation of Capabilities when Transferring Hereditary Information - Unification of Properties of Processes of Nervous System and Formation of Structure of Nervous System According to Certain Rules.

5.3.3. Minimization of Resources Consumption by Means of Optimization of Properties (Set of Subprocesses and Their Properties) of Processes of Nervous System.

5.3.4. Limitation and Evolutionary Determination of Set of Processes (and Their Properties) with which Nervous System of Organism can Interact during the Life Path of this Organism......

5.3.5. Limitation and Optimization of Number of Processes with which Nervous System Interacts Simultaneously.

5.3.6. Minimization of Resources Consumption by Means of Optimization of Functioning of Processes of Nervous System in Order to Ensure Certain Level of TEF Achievement (Satisfaction).

5.4. Optimization of Structure of Interrelationships and Properties of Processes of Nervous System Based on Regularities in Structure of Interrelationships and Properties of Processes of the Universe.

5.4.1. Correlation between Structure of Interrelationships of Processes of Nervous System and Structure of Interrelationships among Processes of the Universe and Their Properties

5.4.2. Hierarchy and Complexity of Structure of Detectors and Generators of Sequences of Signal Combinations.

5.4.3. Parallelism of Structure of Blocks of Analysis/Synthesis of Sequences of Combinations of Signals

5.4.4. Parallelism of Structure of Blocks of Detectors and Generators of Sequences of Combinations of Signals at Every Level of Hierarchy....

5.4.5. Specialization and Localization of Receptors and Outcoming Signals Depending on Type of Interaction with External Processes.

5.4.6. Determination of Structure of Nervous System by Interaction with Processes of the Universe through Sets of Their Properties.

5.4.7. Interrelation in Structure of Parallel Hierarchical Blocks of Detectors and Generators of Sequences of Combinations of Signals as a Result of Interrelation of Different Hierarchical Properties of Processes of the Universe Thanks to which They Interact with Nervous System's Processes.....

5.4.8. Optimization of Number of Detectors and Generators of Sequences of Signal Combinations at Every Level of Hierarchy....

6. Minimal Uniform Process of Nervous System (Structure and Rules of Formation and Functioning Based on Regularities in Interaction of Processes of the Universe)

6.1. General

6.1.1. Similarity of Key Functions of All Processes of Nervous System.

6.1.2. Minimal Uniform Process as Basis of All Processes of Nervous System.

6.2. Rules and Principles of Functioning of Minimal Uniform Functional Process of Nervous

System Based on Regularities in Interaction of Processes of the Universe. 
6.2.1. Optimization of Properties (Set of Subprocesses and their Properties as well as Interrelation and Interrelationships) of Processes of Nervous System that Provide Detection of Interrelated Sequences of Signal Combinations by Interrelated Sequences of Signal Combinations Detected by Them

6.2.2. Variability of those Properties (Set of Subprocesses and Their Properties as well as Interrelation and Interrelationships) of Processes of Nervous System that Provide Detection of Sequences of Signal Combinations

6.2.3. Detection of Interrelation in Sequences of Combinations of Signals only from Processes with which there is Interrelation (there is Direct or Indirect Interaction).

6.2.4. Stability of Interrelation/Interrelationships, through which Detection of Interrelated Sequences of Signal Combinations is Implemented, between Processes of Nervous System and Stability of Their Properties..

6.2.5. Variability of Interrelation/Interrelationships, through which Detection of Interrelated Sequences of Signal Combinations is Implemented, between Processes of Nervous System and Variability of Their Properties.

6.2.6. Combining of Stability and Variability of Interrelationships, through which Detection of Interrelated Sequences of Signal Combinations is Implemented, and Corresponding Properties of Processes of Nervous System.

6.2.7. Signal Generated by Processes of Nervous System as Indicator of Probability of Interaction with Certain Process with Certain Properties and Values of Characteristics.

6.2.8. Signal Generated by Processes of Nervous System as Indicator of Similarity of Process (or Certain Property, or Value of Process Characteristic) with which Interaction is Taking Place Currently to Process (or Certain Property, or Value of Process Characteristic) to whose Detection this Process of Nervous System Adjusted.

6.2.9. Signal Generated by Each Process of the Nervous System as Combination of Indicators of Probability of Interaction with Certain Process (with Certain Properties or Values of Characteristics) and Value of its Similarity to Process (Certain Properties or Values of Characteristics) to Detection of which this Process of the Nervous System Adjusted.

6.2.10. Types of Influences (Signals) of Processes of Nervous System on Other Processes

6.2.11. Dependence of Types of Influences (Signals) between Processes of Nervous System on Interrelation of Processes of the Universe Detected by Them.

6.2.12. Dependence of Characteristics of Interrelationships of Interacting Processes of Nervous System on Statistical Interrelation of Influences Exerted by Them (Signals Generated by Them) and Values of These Influences (Signals) about Accuracy of Processes Determination.

6.2.13. Competitiveness of Interrelationships - Optimization of Relations and Properties of Processes of Nervous System as a Result of Limitation of Their Resources.

6.2.14. Accidental Establishment of New Interrelationships between Processes of Nervous System with Further Strengthening or Weakening/Breaking of These Interrelationships.......44

6.2.15. Optimization of Process of Establishment of Interrelationships between Processes of Nervous System Thanks to Their Additional Interrelation that does not Provide Detection of Interrelated Sequences of Signal Combinations.

6.2.16. Establishment of Interrelationships through which Deactivating Influence is Transmitted between Processes of Nervous System with Their Subprocesses that are Already Receiving Activating Influences from Other Processes of Nervous System. 
6.2.17. Establishment of New Interrelationships between Processes of Nervous System Thanks to Changes in Those Nervous System's Processes that will Exert Influence on Another Process of Nervous System (after Establishment of Interrelationship between Them).

6.2.18. Statistical selection of Process that will be Detected by Certain Process of Nervous System.

6.2.19. Gradual, Multilevel Change of Characteristics of Interrelationships between Processes of Nervous System.

6.2.20. Determination of Adjustment of Processes of Nervous System to Detection of Certain Processes by Given Structure of Interrelation of Processes of Nervous System.

6.2.21. Main and Additional Interrelationships that Transmit Influences on Processes of Nervous System.

6.2.22. Sequential Adjustment of Processes of Nervous System to Detection of Sequences of Signal Combinations.

6.2.23. Optimization of Properties (Set of Subprocesses and Their Properties as well as Interrelationships) of Processes of Nervous System that Provide Transmission of Signal about Accuracy of Process Determination to Other Processes of Nervous System to Ensure Signal Transmission without Distortions and at Required Speed.

6.2.24. Similarity and Difference of Rules of Formation, Strengthening, Weakening and Breaking of Interrelationships between Processes Depending on Type of Influence Transmitted through These Interrelationships

6.2.25. Variety of Types (Specialization) of Processes of Nervous System, Including Minimal Uniform Ones as a Result of Limitation of Nervous System Resources.

6.3. General Structure of Minimal Uniform Process of Nervous System

6.3.1. Sequence of Interrelation of those Subprocesses of Nervous System's Processes that Perceive Influences from Other Processes and Detect Sequences of Signal Combinations.....

6.3.2. Variety of Process Properties and Multivariance of Process Interactions - Parallelism and Branching of Structure of Detector of this Process.

6.3.3. Generated by Process of Nervous System Signal about Accuracy of Determination of Detected Process as Final Sum of All Sequences of Signal Combinations Detected by It......53

6.3.4. Sequence and Branching of Subprocesses that Transmit Signal about Accuracy of Process Determination to Other Processes of Nervous System.

6.3.5. Signal Delay in Interaction of Processes of Nervous System (Process Detection as Long-lasting Process).

6.3.6. Full Structure of Minimal Uniform Process of Nervous System.

7. Structure and Principles of Functioning and Interaction of Main Functional Blocks of Nervous System.

7.1. General.

7.2. General Properties, Principles of Construction and Functioning of Generators and Detectors of Nervous System.

7.2.1. Functions of Nervous System's Processes (Detectors/Generators) Forming Blocks of Analysis and Synthesis of Sequences of Combinations of Signals. 
7.2.2. Processes Detected by Nervous System's Processes that are Included to Blocks of Synthesis of Sequences of Combinations of Signals (Generators) as Processes of Influence of Nervous System's Processes on Other Processes of the Universe.

7.2.3. Difference between Processes Detected by Detectors and Generators of Signals....................58

7.2.4. Advance Detection of Processes by Generators of Sequences of Signal Combinations..........58

7.2.5. Indirect Detection of Processes by Generators of Sequences of Signal Combinations............58

7.2.6. Controlled Formation of Sequences of Signal Combinations Detected by Generators............59

7.2.7. Combination of Nervous System's Processes Exerting Activating and Deactivating Influences, in Detectors and Generators...........................................................................59

7.2.8. Competitiveness of Detectors at Each Level of Hierarchy..................................................59

7.2.9. Sequential Adjustment of Detectors/Generators to Detection of Sequences of Signal Combinations...

7.2.10. Combined Detection of Subprocesses of Detected Process and Transit Signal Transmission.

7.2.11. Complexity of Structure of Individual Detectors/Generators of Sequences of Signal Combinations

7.2.12. Inverse Interrelationships of Detectors/Generators in Blocks of Analysis/Synthesis.............61

7.2.13. Activation of Several Detectors/Generators when Interacting with Certain Process.............62

7.3. System of Formation of Main Signals that Influence Formation of Signal Detected by Generators.

7.3.1. Activation of Nervous System's Processes that Transmit Main Signals to Generators by Signals from Detectors of Processes of Interaction of Organism's Processes with One Another or with Other Processes of the Universe.

7.3.2. Controlled Deactivation of Nervous System's Processes (Primary Generators) that Generate Main Signals to Generators.

7.3.3. Modulation of Interrelation of Nervous System's Processes that Deactivate Deactivators of Primary Generators with Detectors of Processes that Form Current Situation by Signals from Detectors of State and Probability of TEF Achievement.

7.3.4. Relevant Interrelation of Nervous System's Processes that are Included to System of Formation of Main Signals that Influence Formation of Signal by Generators.......................65

7.3.5. Generators as Detectors of the Process that Form Current Situation. .65

7.3.6. Generators as Detectors of the Process that are the Subprocesses of Controlled Processes of Interactions..

7.3.7. Hierarchy of Processes of Influence and Generators and Cascading of System of Formation of Main Signals that Influence Generation of Signal by Generators.

7.4. Principles of Construction and Functioning of Control System of State and Probability of TEF Achievement

7.4.1. Formation of Interrelationships that Transmit Influence to Unblocking Deactivators under Influence of Integrated Signal from Relevant Detectors of State and Probability of TEF Achievement.....

7.4.2. Formation of New Detectors of State and Probability of TEF Achievement in Nervous System of Combined Type.

7.4.3. Sequence of Formation of New Detectors of State and Probability of TEF Achievement .68 
7.4.4. Formation of Interrelationships through which Influence is Transmitted on Selfadjusting Detectors of State and Probability of TEF Achievement under Influence of Integrated Signal from Relevant Detectors of State and Probability of TEF Achievement.

7.4.5. Structural Hierarchy of Detectors of State and Probability of TEF Achievement.

7.4.6. Activating and Deactivating Influences of Primary Detectors of State and Probability of TEF Achievement on Secondary Detectors of State and Probability of TEF Achievement.

7.4.7. Generators as Detectors of Processes whose Implementation Can Lead to Increase in State and/or Probability of Achievement of TEFs (Self-adjusting Primary Detectors of State and Probability of TEF Achievement).

7.4.8. Sequence of Activation (Generation of Signals About Accuracy of Determination of Processes) of Primary Detectors of State and Probability of TEF Achievement when Generating Sequence of Signal Combinations.

7.4.9. Correlation between Sequences of Signal Combinations Generated by Generators and Primary Detectors of State and Probability of TEF Achievement.

7.4.10. Inverse Deactivating Influences on Processes of Control of Probability of TEF Achievement.

7.5. System of Optimization of Implementation of Controlled Processes of Interaction (Smoothing).

7.5.1. Overload and Overregulation when Implementing Controlled Processes of Interaction......

7.5.2. Inability of Guaranteed Adjustment of Generators to Generation of Sequences of Signal Combinations that will not Lead to Overload and Overregulation under Action of Modulating Signals from Detectors of State and Probability of TEF Achievement......

7.5.3. Basic Rules of Functioning and Principles of Construction of Nervous System's Processes that Help to Avoid Overload and Overregulation when Implementing Controlled Processes of Interaction.

7.6. Additional Types of Interaction of Processes of Nervous System.

7.6.1. Types of Interaction between Preprogrammed and Self-programming Processes of Nervous System.

7.6.2. Modulation of Adjustment of Self-adjusting (Self-programmed) Processes of Nervous System (Detectors) to Detection of Processes of the Universe during Detection of which State and Probability of Achievement of TEFs Increase.

7.7. Derivative Properties of Interaction of Processes of Nervous System

7.7.1. Dependence of Generated by Process of Nervous System Signal about Accuracy of Determination of Process of the Universe on Signals of Nervous System's Processes that Detect Processes of the Universe Interrelated with the Specified Process of the Universe, Including on Relevant Generators.

7.7.2. Indirect Detection and Static and Dynamic Drift when Generating by Processes of Nervous System Signals about Accuracy of Process Determination.......................................76

7.7.3. Advance Indirect Detection of Processes of the Universe.

7.7.4. Change and/or Formation of New Interrelationships between Processes of Nervous System through Advance Indirect Detection of Processes of the Universe... 
7.7.5. Dynamic Linking of Nervous System's Processes that Detect Dynamically (Indirectly through Sequence of Interactions with Other Processes within Hierarchically Higher Processes) Related Processes of the Universe

7.7.6. Advance Detection and Dynamic Linking as Main Processes of Interaction of Processes of Nervous System during Their Interaction with Processes of the Universe.

7.7.7. Multiple Interrelation of Processes of Nervous System.

7.7.8. Long-lasting Interaction of Processes of Nervous System as a Result of Indirect Detection (Indirect Generation of Signals about Accuracy of Process Determination).

7.7.9. Mixed Interaction of Processes of Nervous System as a Result of Indirect Detection and Direct Interaction with Processes of the Universe.

7.7.10. Increase in Current Accuracy (with Current Interaction) of Detection of Processes With Increasing Number of Cycles of Interaction of Processes of Nervous System with Processes of the Universe in Current Situation.

7.7.11. Increase in Accuracy of Adjustment of Processes of Nervous System to Detection of Processes of the Universe With Increasing Number of Interaction with These Processes in Different (Similar and Dissimilar) Current Situations.

7.7.12. Increase in Accuracy of Adjustment of Processes of Nervous System to Detection of Processes of the Universe Thanks to Interaction of Processes of Nervous System through Existing Adjusted Interrelationship in Absence of Direct Influences from Receptors on Processes of Nervous System.

7.7.13. Change and/or Formation of New Interrelationships between Processes of Nervous System without Their Direct Detection when Interacting with Processes of the Universe.....83

7.7.14. Dependence of Conformity of Changes of Interrelationships of Processes of Nervous System to Existing Interrelations among Processes of the Universe (at Change or Formation of These Interrelations without Direct Interaction with Corresponding Processes of the Universe) on Previous Cycles of Interactions of Processes of Nervous System with Processes of the Universe

7.7.15. Processes of Organism - Processes of the Universe with which Nervous System's Processes that Belong to this Organism Most Frequently Interact.

7.7.16. Dependence of Long-lasting Interaction of Processes of Nervous System as a Result of Indirect Detection of Processes of the Universe on Detectors of State and Probability of TEF Achievement

7.7.17. Dependence of Signals Generated by Processes of Nervous System during Longlasting Direct Interaction and Interaction with Indirect Detection from Signals Generated by Them Earlier within this Interaction.

7.7.18. Long-lasting Interaction of Processes of Nervous System as a Result of Indirect Detection of Processes of the Universe as Main Process of Interaction of Processes of Nervous System in Nervous System of Certain Level of Complexity.

7.7.19. "Self-awareness" and "Awareness" of Processes of the Universe as a Result of Interaction of Processes of Nervous System in Process of Their Long-lasting Interaction as a Result of Indirect Detection of Processes of the Universe. 


\section{Preface}

From an early age, two things fascinated me: the structure and laws of the Universe, and the mystery of the mind. Studying the history of scientific discoveries made in the past, I saw that an important role in these discoveries is played by the availability of the necessary tools, understanding of the principles of its work and, accordingly, skillful use of it. And one of the most important tools among others was the researcher mind. This idea has set priorities in my research - for a deeper understanding of the physical laws of the universe, it is first necessary to understand this most important research tool. What is the mind, how and why it arose, what are the basic principles of its structure and functioning. And then, after finding the answer to these questions, it will be easier to cope with the second - a deeper understanding of the physical laws of the universe.

From the beginning, I did not limit myself to any particular approach, looking for answers in different areas, where clues could be scattered: religion, art, history, psychology, biology, neuroscience, physics, computer science, engineering. Unfortunately, the study of the sources available at that time (primarily in psychology and neuroscience) did not provide the desired answer to the questions. The picture, which consisted of the available data, was not complete, with large gaps, painted either with too small strokes, or very large and blurred. In addition, these strokes often contradicted each other and did not make it clear what kind of object they reflect. Therefore, this path, in my opinion, required further research that would reveal more of important details of the structure of our brain and clarify some of the already known. However, experience in engineering has shown that even after completely disassembling an unknown device and studying in detail all of its elements, it is not always possible to understand the general principles of its structure and functioning. And even if it succeeds, the use of this approach will not necessarily lead to an answer to the question of the causes of emergence and development of the brain. But what if use a different approach to solve this problem. Instead of trying to understand the structure of the brain through its direct study, make an attempt to construct it. I. e. by logical constructions, based on certain initial assumptions, to analyze what conditions are necessary for the emergence of the brain and related phenomena, what causes may lead to the possibility of its emergence, what are the main factors determining its structure and function, and what are the basic principles of its structure and functioning. As initial assumptions I chose the following:

- causality as the basis of interaction of elements of the universe;

- live organisms as one of the possible forms of existence of matter;

- evolutionary development of living organism.

Also, to describe the universe in which the emergence and development of intelligent life is possible, the concept of process (process of the universe) was used. This concept was formed as a necessary universal unit of the universe, the properties of which can simultaneously ensure its functioning as a basis for the emergence, evolution and functioning of intelligent life, and also provide an opportunity for intelligent life forms during different stages of their evolution to perceive this universe and interact with it using the same universal principles. The properties of the processes of the universe are described in detail in the section 3.1.

The result obtained during the implementation of the above approach is summarized in this paper.

This work is divided into seven chapters. Chapters 1 and 2 are brief introductory sections which summarize the main purpose and approaches used in this research. Chapter 3 describes the basic properties of the universe and living matter and is basic for all subsequent chapters. The statements of this chapter were used in the following chapters as a basis for further analysis. This chapter itself, in turn, was formed and supplemented in the analysis of the issues covered in the following chapters. Chapter 4 to 7 are divided into paragraphs, each of which covers the analysis of a particular issue. The analysis covered in each paragraph is based on the results of the analysis covered in the previous paragraphs or on the statements set out in Chapter 3. Chapter 4 describes the general causes that could lead to emerging of nervous system, its main functions, the principles of its interaction with other systems of organism and the environment, and a description of the basic block diagram of the nervous system. Chapter 5 analyzes the general principles of formation, limitations and structure of the nervous system. Chapter 6 describes the minimum functional element of the nervous system and the principles of its functioning and structure. 
Chapter 7 is devoted to a more detailed description of the structure and functioning of the main blocks of the nervous system, defined in the previous chapters, and the feauture of their functioning and interaction, including those that lead to such effects as higher nervous functions. 
The development of our civilization, consciousness, spirit, views, knowledge is only the continuation of development of the living matter which, in its turn, is the form of organization of the nonliving matter.

But how incredible and beautiful this continuation is... More to come!!!

Only the life itself is able to give the reason for living.

\section{Research Objective and Methods}

The objective of this research is to determine the common causes of development and principles of forming and functioning of subsystems that implement the intellectual properties of the system to which they belong.

As part of the research, the system construction approach was used where the system would have the capability and practicability (in the context of maintenance of organism's life to which it belongs) of emergence and existence and would ensure the implementation of the intellectual properties of the living organisms under the constraints and opportunities available in nature during its implementation.

\section{Basic Assumptions Accepted during Construction}

The initial assumptions set out in the preface (causality as the basis of interaction of elements of the universe; live organisms as one of the possible forms of existence of matter; evolutionary development of living organism) can be extended by additional statements that clarify these initial assumptions and are their consequence:

- the emergence and development of life is possible only in the universe, the physical laws of which meet certain criteria, due to which it is possible the emergence and existence of intelligent life;

- the nervous system arose and develops due to the evolution of living organisms;

- the functions of the nervous system of living organisms determined by their ability to ensure the survival of these living organisms in the process of their evolution;

- capabilities and structure of the nervous system determined by the physical properties of the universe where life is possible;

- the activity of the nervous system and all the phenomena that emerge as a result of this activity, caused and determined solely to physical processes that take place in the nervous system.

As a result: each and all intellectual processes inherent in intelligent creatures are the manifestation of the work of the nervous system that was formed as a result of the living matter evolution, are based on its properties, which are the derivatives from the universe properties, and the universe properties are the ones that can ensure the emergence and existence of intelligent life. 


\section{Initial statements and definitions}

\subsection{Properties of Environment (Universe) in which Intelligent Life Develops}

The environment (the universe or its part) where the intelligent life exists consists (at a certain initial level of abstraction) of the set (sets) of the identical (same-type) interacting with one another elements. Each of such elements is an interrelated set of properties - characteristics each of which has its set of allowable values and functions of interdependencies between the values of these characteristics. The interaction of the elements with each other is manifested in the interdependence between some (different) characteristics of the interacting elements. The interaction of the elements and the change of the interaction are determined by the functions of the values of their corresponding characteristics that cause and determine this interaction of the elements. To each of the properties that cause and determine the interaction of the elements corresponds certain functions of the interdependence of a certain set of characteristics of the interacting elements. The properties of the elements - the set of the characteristics, allowable values of these characteristics, the set of functions that determine the interdependence of the characteristics (both within the limits of one element and in case of interaction of the elements) - have to remain unchanged during the long-lasting number of cycles of change of the values of the element characteristics (sufficient for the emergence and development of intelligent life in the specified environment).

Each of the elements of the set (sets) described above shall have such a set of the properties so that it would be possible in the environment created on the basis of the interaction of these elements to create the sets of the identical (same-type) and similar, localized, long-lasting hierarchical processes, which are the processes of the change of the characteristics of the environment initial elements according to their functions of interdependencies (both within the limits of one element and in case of interaction of the elements). In such a case, each hierarchical process at every level of the hierarchy will be a set of the interrelated properties and will be able to interact with other processes creating the hierarchical processes of the higher level of the hierarchy. The environment elements themselves may also be considered as the processes of the zero (initial) level.

As the processes of the higher level of the hierarchy are the processes of the interaction of the processes of the lower level of the hierarchy (hereinafter subprocesses), the properties of the processes of the higher level of the hierarchy - the characteristics of the processes, the values of the characteristics of the processes, the functions of the interdependencies of these characteristics (both within the limits of the process and within the limits of the interaction of this process with other processes) - are the result of the interaction of the processes of the lower levels of the hierarchy (subprocesses) according to their characteristics, the values of these characteristics and the functions of interdependence of the characteristics of these processes (both within the limits of an individual process and within the limits of the interaction of each process with other processes).

The interaction of the processes takes place as a result of their interrelation - such properties (the set and values of the characteristics that cause and determine their interaction, and also the functions that determine this interaction) that can lead to the interaction of these processes with each other or to their interaction with the same ones, third processes interrelated with them. The structure of the interrelation of the processes determines which processes may interact with one another and in which order. The magnitude of the interrelation of the processes determines the probability and the magnitude (interdependence of the properties of the interacting processes) of the process interaction.

The long-lasting (during several cycles of interaction), continuous interrelation of two processes that leads to their interaction at which unidirectional or bidirectional interdependence of their properties is implemented will be hereinafter referred to as the "interrelationship (relation, connection) between the processes".

The independent interaction of the group of the processes, as a result of which the influences are implemented (directly or indirectly, through the chain of the processes interaction) on one and the same 
process, will be hereinafter referred to as the simultaneous one (from the point of view of the specified process) if this process, as a result of the specified interactions, receive such influences (which are the result of these interactions) each of which does not precede and does not follow the other one and, thus, cannot influence the specified process earlier or later than other influence.

The localization of the hierarchical processes is manifested in that the interrelation and, thus, the interaction among the processes that form the process of a certain level of the hierarchy will be higher than the interaction of these processes with other processes of the environment. Thus, the processes included to a certain hierarchically higher process, during the most number of the interaction cycles and with a bigger values of the interdependence of the characteristics, will interact with other processes included to the same hierarchically higher process rather than with the processes that are not included in it.

The functioning or the interaction of the processes of all hierarchy levels results in the changes (can be cyclic) of the properties of these processes or the values of their characteristics and, therefore, in their transformation to other processes as a result of the change of the properties and/or interaction of the hierarchically lower processes. The difference of each process of the universe from other processes lies in the difference of its properties and/or values of characteristics. The processes that have the same set of properties are the identical (same-type) processes. The identical processes in the universe differ by the values of at least one of its characteristics. The processes that have certain differences in the set of the properties are the similar processes. The level of their similarity depends on the ratio of the different and same properties between the processes being compared. The higher the processes hierarchically are (the more the number of the processes of the initial level is included to them), the higher the probability of presence of the similar and dissimilar processes in them is. I.e. the higher the process hierarchy level is, the higher the probability of the similarity is (from complete dissimilarity to complete similarity identity), and not the identity of these processes. The processes of the different hierarchy levels, when changing, transform to other processes that are identical (same type) or similar to themselves prior to changing.

As the environment elements, which are the steady set of the properties - the characteristics with unchangeable allowable values and the set of the functions that determine the interdependence of values of these characteristics (both within the limits of one element and in case of interaction of the elements) lie at the root of all hierarchical processes, such steadiness in the hierarchical processes themselves results in the steadiness of the rules of the change of the properties (the values of the characteristics) or the rules of the transformation (changes of the properties themselves and/or the set of the properties) of these hierarchical processes. Accordingly, these rules (cause-and-effect relations) themselves are determined (are a manifestation) by the properties (set of the properties and values of their characteristics) of the specified hierarchical processes.

Thus, the universe where the intelligent life exists is the environment consisting of the set (sets) of the processes that can, interacting with one another, form the sets of the hierarchical processes that function and interact on the basis of the unchangeable (at least for the time of existence of this life form) rules cause-and-effect relations. These rules of interaction are determined by the set of the properties of the specified hierarchical processes.

\subsection{Life and Its Key Properties}

Life is such a form of the matter existence that is a group of the interacting processes for whose functioning and interaction a constant influx of resources from the outside environment is required, and the nature of their interaction ensures obtaining of this resources, the long-lasting functioning of this group of the processes and/or the reproduction and functioning of the similar (3.1) to them groups of processes.

The groups of such interrelated processes, which completely realize the above specified functions, form the quasi-closed (localized) systems - living organisms. The minimal group of such processes forms a minimal living organism — a living cell.

Generally, the resources for a certain process that is included to the group of the interacting processes 
that form a living system (a living organism) are the processes (both external with respect to this organism and internal, i.e. the subprocesses of this process - the internal resources of the process) with such properties and values of the characteristics that can, as a result of the interaction of these processes or subprocesses (3.1), influence the functioning of the specified process promoting the implementation of its functions within a living organism.

The external resources (the resources from the outside environment) for the groups of the interacting processes forming a living organism may be divided into two types: the energy (the influence from other processes of the universe as a result of which certain characteristics of the processes of this group change that ensures their further functioning in the form of the specified group) and the construction material (other processes of the universe that may be included by the processes of the specified group to this group to create the new ones, to support the functioning or to change the existing processes of this group).

Each living organism, within a certain limited period (within a limited number of the process interaction cycles) of its existence, can get a certain limited set of resources in the environment of its existence depending on the set and properties of the processes that form this living organism and the set and the properties of the processes of the universe (the outside environment) with which they can interact within a certain limited period (3.1).

The long-lasting functioning of the group of the interrelated processes forming the process (3.1) that is a living cell is ensured through the specific functional interaction and implementation of certain critical for these functions by them - target evolution functions (TEF):

- interaction with other processes of the universe in order to obtain the external processes or energy required for the implementation of the functions of the group of the processes;

- control over the processes that take place in the group - ensuring the interaction of the subprocesses that form this group in order to maintain the long-lasting functioning of the group, to reproduce the processes required for ensuring the functioning of the system, to transform into other ones or to exclude from the group the processes that interfere with the group functioning;

- making changes in the processes of control and reproduction of other processes of this group mutation as a way to ensure the diversity of groups (the greater the diversity, the better chances are that any of the groups will continue existing);

- reproduction of the similar groups of the processes (propagation) as a way to ensure the preservation of living systems thanks to a large quantity (the more there are, the better chances are that any of the groups will continue existing);

- accumulation of changes and transfer (during reproduction) of the processes that ensure the implementation of control and reproduction of other processes of this group - hereditary mechanism (thanks to the hereditary mechanism, reproduction, mutations, and process of natural selection it becomes possible to accumulate those changes in the generations of the groups of the processes, which form the living organisms, that allow them to implement their target evolution functions more efficiently and effectively);

- influence of the parent group (the group that implemented its reproduction) on other processes of the universe and/or the processes of the groups that are its derivatives in order to provide better possibilities for the implementation of the target evolution functions by these groups of the processes that are its derivatives.

As a result of mutations and the natural selection, new organisms can emerge that are formed by the group of the interacting processes (living cells) - multicellular living organisms. These living organisms function by implementing the same target evolution functions as the unicellular organisms both at the level of each cell and at the level of the whole group of cells. In its turn, during the evolutionary development of the multicellular living organisms, the formation of the groups (assemblies) of the similar multicellular living organisms is possible. These groups (assemblies) also function by implementing the same target evolution functions as the multicellular organisms as at the level of individual cells and at the level of a multicellular organism and the group of similar multicellular organisms. Due to greater complexity of the process that is a multicellular living organism or the group of multicellular organisms the complexity of its possible interactions with other processes of the universe (those that are not included to the group of the processes that form this organism or the group of the processes that form this 
assembly) increases as well. Thus, additional functions can emerge that, thanks to the possibility of a more complicated interaction of the processes of a living organism (in a multicellular organism) or individual multicellular living organisms (as an assembly) with one another and with other processes of the universe, can ensure a more efficient and effective implementation of the above specified target evolution functions of a multicellular living organism or an assembly of living organisms.

Taking into account the constant competition among the living systems and the constant change of the environment of their existence throughout the course of life development history, as a result of mutations, the living organisms change towards optimization for a more optimal in the context of utilization of resources and more efficient and effective (complete) implementation of the above specified functions in the environment of their existence.

The listed above target evolution functions can be grouped and designated as follows: survival, propagation, ensuring offspring survival. Every form (species) of living organisms has to implement these target evolution functions. All forms (species) of living organisms that implement these target evolution functions less efficiently and less effectively than other forms (species) of living organisms that require the same external resources for their existence, are pushed out (disappear) by other, more efficient and effective forms (species) of living organisms as a result of competition in course of time. All systems of a living organism that do not contribute to achieving TEFs disappear in the subsequent generations of this form (species) of the living organism (in order to minimize the organism's expenses for their functioning) as a result of intraspecific competition of the living organisms.

\section{Key Functions, Principles of Interaction and Simplified Schematic Diagram of Nervous System}

\subsection{Basics of Functioning and Approaches to Construction of Nervous System of Living Organism}

Any system of a living organism has to contribute to at least one of the target evolution functions: better survival of the organism, the ability to give offspring, the ability to ensure better survival of the offspring (3.2).

\subsubsection{Living Organism as Holistic System of Subsystems Interacting in Coordinated and Targeted Manner}

A living organism is an group of hierarchically interrelated processes (3.2, 3.1). It exists by interacting with the external towards itself processes of the universe that directly or indirectly (interacting with other processes that eventually interact with the end process) influence it $(3.2,3.1)$. If the processes of a living organism interact with one another in a coordinated way to achieve the TEFs of this organism, depending on their current state and the current state of interaction with the external processes, their efficiency and effectiveness in achieving the target evolution functions will be higher than the one of other similar organisms that will not have such a coordinated interaction of their subprocesses. In this case, a higher efficiency and effectiveness can be achieved through the possibility of an optimal utilization of resources of internal and external (with respect to a living organism) processes when achieving the target evolution functions and the enhancement of the possibility to obtain new external resources for achieving the target evolution functions.

If, when implementing the interaction, its character is influenced not only by the current (instant) state of interaction of the processes of the organism and the external processes, but also by the possible in the future (through several interactions) state of interaction, the efficiency and effectiveness of such living organism in achieving the target evolution functions will be even higher thanks to taking into account the interaction of a bigger number of the processes that can influence the TEF achievement in the process of their achieving by the living organism. The bigger the number of the processes and their properties will be taken into account in this case and the further in time (through a longer sequence of interactions) the state 
of their interaction will be calculated, the more the efficiency and effectiveness of an organism can be.

The forecast of the possible in the future state of interaction (in the absence of the possibility of its direct perception) is possible, if it is possible to determine the processes (the set of the processes, their properties and the values of characteristics) that interact at a certain point (have such properties and values of characteristics that lead to their local interrelation), and if the rules of transformation (cause-and-effect relations - 3.1) of these processes to a certain result (the set of the processes, their properties and the values of characteristics) in the future after the interaction are known. In the universe, where these rules are unchangeable for one and the same processes (3.1), it is possible to determine the transformation rules based on the data about the interaction of these processes in the past. It is necessary for this, in case of interaction of the group of the processes, to determine their properties and values of their characteristics prior to the interaction and the properties and values of the characteristics of these processes after the interaction. In case of interaction of these processes in the future, it is possible to substitute (activate) at once the previously determined result and, thus, to foresee the result of the current interactions prior to its direct achievement. A more complicated and far-reaching forecasting can be possible by means of combining the sets and the sequences of such transformation rules for a certain group of processes.

\subsubsection{Enhancement of Efficiency and Effectiveness in Achieving TEFs through Change of Properties of Living Organism's Processes}

The processes of the organism, at a certain point of time - in a certain situation (hereinafter referred to as the situation - the process that is the set of the interrelated processes of the universe, including the organism's processes, with certain properties and values of characteristics that, when interacting with one another, can, directly or indirectly, to different extents, influence the organism's TEF achievement), can interact with one another and with other processes of the universe according to the rules that are determined by the set of their properties and values of their characteristics (3.1). In order for the living organism's processes to be able to enhance the efficiency and effectiveness of the interaction with one another, in a certain current-foreseeable situation (the current-foreseeable situation is a combination of the current situation and the foreseeable, future, after a certain number of cycles of the interaction of the processes that form the current situation, situation - 4.1.1), it is necessary that at least a part of the processes of the living organism could change their properties and values of characteristics so that the rules of their interaction with other processes in this situation change. As these changes must lead to the enhancement of the efficiency and effectiveness of achieving TEFs of the organism, they must, therefore, depend on the fact to what extent the organism's target evolution functions are implemented with the specified changes of the properties and the values of characteristics of the organism's processes in a certain current-foreseeable situation.

The determination of the fact which properties in which processes of the living organism and in which way are to change can be done randomly (by random changes of available properties) and/or according to certain rules if these rules were defined beforehand by previous random changes of the set of the subprocesses of living organisms, their properties or values of their certain characteristics and are implemented, therefore, with the help of this changed set of the processes or the changed properties or the values of characteristics of the living organism's processes.

Those changes that lead to more efficient and effective (decrease in number of unachieved TEFs and increase in number of achieved TEFs and/or increase of the level of their achievement in a current or future, after a certain number of cycles of process interaction, situation) interactions of the processes of the organism with one another and with the external processes in a certain current-foreseeable situation must have a higher probability of preservation than those that lead to less efficient and effective interactions. As the rules of interaction of processes in the universe are unchangeable (3.1), an organism in which the properties and the values of the characteristics that previously resulted in more efficient and effective interactions in a certain situation will have been preserved will have a higher probability of the same efficiency and effectiveness of achieving TEFs (in a situation similar to the specified one) in the future than the organism, in which such changes of the properties and the values of the characteristics of its processes will not have been preserved. Moreover, the more similar this future situation (the set of the processes, their properties and the values of their characteristics) to the previous one, in which the efficient and effective interactions were achieved, is and the more similar in this situation the interaction 
of the processes of the organism with one another and with other processes is, the higher the probability will be for the same efficiency and effectiveness of achieving TEFs in the future situation as was in the past. A better adaptation of a living organism to existence in a certain environment is realized thanks to accumulation of such changes.

Hereinafter, the terms "satisfied TEFs"/"dissatisfied TEFs" can be used together with the terms "achieved TEFs"/"unachieved TEFs", respectively. The term "level of TEF achievement" is equal to the terms "level of TEF satisfaction"/"level of TEF dissatisfaction". Besides, the term "determination of TEF achievement in a certain situation" means the simultaneous determination of the current state of TEF achievement in the current situation and the determination of the probability of achievement of a certain state of the TEF achievement in a foreseeable future situation. When using the terms "current situation", "specified situation" or "situation" for the purposes of description of the functions of the processes of the organism that ensure the efficient and effective interaction of all organism processes in order to efficiently and effectively achieve TEFs of the organism, these terms mean the current-foreseeable situation.

\subsubsection{List of Key Functions of Nervous System of a Living Organism}

Thus, according to the above $(4.1 .1,4.1 .2)$, a living organism will be able to enhance the efficiency and effectiveness of achieving its target evolution functions if all its processes or a certain group of processes perform the following functions:

- determination of the processes (the list of the processes, their properties and the current values of their characteristics) that interact at a certain point of time and whose interaction has influence the achievement of the target evolution functions by the organism;

- determination of the sequence of changes of the determined processes (subprocesses) during their functioning or interaction (relations between the current state of the processes and their state after the interaction [change] — the results of interaction/change of the processes) - determination of the causeand-effect relations during change and interaction of the processes of the universe;

- influence (direct or indirect) other processes the interaction with which influence the achievement by the organism of its TEFs depending on the current conditions (the current and foreseeable list of the processes and the values of their characteristics) in order to change the course of their interaction with one another and with other processes in such a way that to ensure such a course of interaction of all processes that will lead to a more efficient and effective achievement of the target evolution functions by the living organism in the whole;

- change of the properties and values of characteristics and preservation of those that led to such interaction of the processes that ensured a more efficient and effective achievement of the target evolution functions under certain determined conditions (the current and foreseeable list of the processes, the values of their characteristics and the influences exerted on the processes).

The efficiency and effectiveness of the organism in achieving its target evolution functions during interaction of all its processes with one another and with the external (with respect to this living organism) processes of the universe will depend on the fact to what extent one or another function of the listed ones is implemented in this living organism.

In organisms of rather high complexity, consisting of many processes and for which the speed of interaction with external processes is critical, a separate specialized system of interrelated (3.1) hierarchical processes - the nervous system - can arise (by evolution) for the implementation of the above functions. Depending on the complexity of the nervous system, every process that forms it can perform all functions or (in more complicated systems) have the prevailing specialization in performing the specific functions. In case of such a specialization, the functions performed are divided into the subsystems (groups of processes with the similar functions) of the nervous system.

According to the above, the nervous system of an organism is a hierarchical process that is a set of the interrelated hierarchical processes (processes of the nervous system). These processes, by perceiving external and internal (with respect to the organism) processes (their properties and values of characteristics), the order of their change and interaction (cause-and-effect relations) and exerting the 
influence on them, interact with each other and change their properties and values of characteristics according to such rules that in the future situations, similar to the past ones, to determine (detect), perceiving these external and internal processes, those of them (properties, the values of characteristics and cause-and-effect relations) whose influence was, in the past situations, important for the achievement by the organism of its TEFs and, thus, to create (generate) and to exert such influences on these processes that, under the conditions similar to the future current ones (the current situation and the foreseeable, future situation), led to such interaction of the processes in the past that led to the efficient and effective (4.1.2) achievement by the organism of its TEF and, thus, is very likely to lead to the efficient and effective achievement by this organism of its TEFs, in case of exertion of these influences, in the current situation (the current situation and the foreseeable, future situation) in the future.

In the nervous system, in addition to the processes that are directly involved in the implementation of its functions ( primary processes of the nervous system), there can also be the processes that do not directly implement these functions, but ensure the functioning of the nervous system's primary processes - the secondary processes. Only the nervous system's primary processes will be considered hereinafter.

\subsection{Interaction of Nervous System with External Processes}

\subsubsection{General}

According to the original assumptions (3.1), the universe where the intelligent life exists consists (at the initial level of abstraction) of the set (sets) of the identical (same-type) elements that can form the sets of the hierarchical processes that interact with each other in order of their interrelation changing within these interactions the values of characteristics of one another. Thus, every process in the universe manifests itself with respect to another process through a direct or indirect (by interacting with other processes that eventually interact with the end process - 4.1.1) interaction with it.

According to the original assumptions (3.1), any process in the universe is a set of interrelated properties with certain values of characteristics. Besides, all processes of a certain level of the hierarchy (except for the initial one) in the universe consist of the processes of the lower level that are more interrelated to each other and, thus, during the long-lasting number of cycles of interaction with the bigger value of interdependence, interact with one another rather than with other processes that are not included to this process of the upper level. Thus, this interrelation of the properties of the process and the interrelation of the processes of the lower level (for the processes of a certain level of the hierarchy, except for the initial one) will be reflected also in the interaction of this process of a certain level of the hierarchy with other processes of the universe.

During a direct or indirect interaction of two processes of a certain level of the hierarchy, the value of one or several characteristics for one or several subprocesses that form each of these two interacting processes can change in each of these processes (3.1). The value of change of these characteristics depends on the properties of the interacting processes - the initial values of characteristics and functions of interdependence of characteristics (3.1).

Thus, the manifestation of one process of a certain level of the hierarchy for other process of a certain level of the hierarchy will lie in the change of the values of certain characteristics of this other process by a certain value. Moreover, this change of the values of characteristics of other process - the value, the combination, the sequence, and the interrelation of the value, the combination and the sequence of these changes - will depend, in addition to the properties of other process, also on the unique for the first process set of its interrelated properties, values of characteristics and interrelation of its subprocesses (processes of the lower level of the hierarchy) and their interrelated properties.

Thus, the determination by the nervous system's processes of the external with respect to themselves processes (the list of the processes, the list of their properties and the values of characteristics at a certain point in time of their interaction) and the rules of their change lies in the change of the values of the characteristics of the nervous system's processes as a result of their direct or indirect interaction with the external processes. Therefore, the influence of the nervous system's processes on the external (with respect to themselves) processes lies in the change of the values of the characteristics of these external 
processes as a result of their interaction with the nervous system's processes.

The processes of interaction of the nervous system's processes thanks to which the functions of the nervous system are implemented will be hereinafter referred to as the processes of signal transmission, and the influence of one process of the nervous system on the other process within the process of signal transmission - a signal.

\subsubsection{Perception of External Processes as Corresponding to Them Sequence of Signal Combinations - Changes of Characteristics of Certain Processes of Nervous System}

The current external (with respect to the nervous system) processes will be determined by the certain processes of the nervous system as a result of the direct or indirect interaction of these external processes with them (4.2.1). At each particular point of time of such interaction the external processes, directly or indirectly, influence (interact) one or several (set) processes (receptors) of the organism's nervous system changing their certain properties (the values of certain characteristics) - state — by activating them. And in each such case, at a certain point of time, the combination and the value as well as the interrelation of the combination and the value of change of characteristics of these receptors for this unique external process will be unique, will depend on the certain properties and values of characteristics of this process and their interrelation, and, thus, will reflect them (4.2.1). These combinations will be in some way similar for one and the same process at different points of time (in its different states of similarity to the previous state) or for the similar processes (3.1). The change of the process (the change of the values of characteristics, the change of the properties and the values of characteristics during several cycles of interaction of the processes that are hierarchically lower than it, the interaction with other processes etc.) will be detected by the nervous system as the change of combinations (the list of activated receptors and the value of their activation) of activated receptors. As the changes of the processes are made according to the unchangeable rules that work in the universe (3.1), the detected sequences of combinations of activated receptors will be, therefore, unchangeable (similar) and unique (in certain aspects) for every individual unique rule of interaction (change) of the processes in the universe. Thus, the nervous system detects the processes of the universe as the set and the values of characteristics and the rules (functions) of their interdependence (cause-and-effect relations) during change or interaction of the processes in the form of the unique combinations of change of certain characteristics of receptors and the unique sequences of such combinations. Moreover, this uniqueness will lie in the unique interrelation of the values, the combination and the sequence of changes of the specified characteristics.

\subsubsection{Influence on External Processes by Generating Sequences of Signal Combinations - Changes of Characteristics of Certain (Those that can Influence External Processes) Processes of Nervous System}

The current influence (the control signals) on the external (with respect to the nervous system) processes is exerted by a certain set of the processes of the nervous system through a direct or indirect change of the values of some characteristics of these external (with respect to the nervous system) processes. Such a change of the values of characteristics of these processes will lead, in its turn, to the change of the course of the specified processes and the character of their interaction with other processes according to the properties of these processes (3.1).

In a complex organism that is a set of many processes and that interacts simultaneously with many external processes that can also be the subprocesses of the hierarchically higher process, the nervous system has to control (in order to ensure a more efficient and effective achievement of TEFs of the organism - 4.1) simultaneously many external (with respect to the nervous system) processes. Therefore, in a certain situation, it will perform a certain set of combinations of such influences - produce a certain set of combinations of control signals.

It is possible to determine beforehand which signals in the set of all possible combinations of control signals will lead to the achievement of the predetermined result only in case if the rules of interaction (cause-and-effect relations) between the processes of the universe are unchangeable (4.1.1). In such universe, if the same groups of the processes are in the same situation (the set of the processes, the set of 
their properties and values of their characteristics - 4.1.2), the interaction between them will be performed every time in the same manner, in the same sequence, and will lead to the same result (the set of the processes after the interaction, the set of their properties after the interaction and the values of their characteristics). A more complex result can be obtained thanks to the realization of a more complicated (hierarchically higher) process that will be a combination of the arrays of sequences of several interactions of hierarchically lower processes (every interaction with its intermediate result). Therefore, if, in case of the group of the processes that are in a certain situation (the set of the processes, the set of their properties and values of their characteristics), it is necessary to obtain the predetermined result, it is necessary to exert such an influence on these processes that they interact in the sequence, in the combination and with the change of the values of their characteristics that lead to this result. Moreover, to obtain one and the same result for one and the same unique set of the processes, in one and the same unique situation, it is necessary to perform one and the same unique sequence of combinations of influences. And this uniqueness will lie in the unique interrelation of the values, the combination and the sequence of these influences.

Thus, to achieve the required result, the nervous system must produce, in a certain situation, such sequences of combinations of control signals to other processes that will lead to such changes of the values of characteristics of these processes and in such a sequence so that they (through the changes of the values of their characteristics) interact with one another and with other processes in such a way and in such a sequence so that the predetermined result is eventually obtained. The change of the sequences of combinations of control signals and their characteristics in a certain situation will lead to obtaining of another result in this situation. The optimization of combinations of sequences of control signals and their characteristics to ensure the increased probability, efficiency and effectiveness of achieving by the organism its target evolution functions (TEFs) will be performed thanks to the mechanism of change and preservation of the properties of the nervous system's processes under the principle described above (4.1.2, 4.1.3).

\subsection{Minimal Block Diagram of Nervous System Structure}

Taking in consideration the above $(4.1,4.2)$, the minimal macrostructure of the nervous system can be determined. Thus, the nervous system has to include the subsystems (see fig. 1) that would be able to perceive (receptors) and to analyze the available combinations of signals and their sequences (blocks of analysis of sequences of combinations of incoming signal). The system also has to include the subsystems that would synthesize the required combinations of control signals (reactions) and their sequences (blocks of synthesis of sequences of combinations of outcoming signals). The control signals shall be part of such sequences of signal combinations that lead to the increased probability, efficiency and effectiveness of achievement of target evolution functions in a certain situation (4.1.2). Therefore, the nervous system has to include the subsystems that would detect the processes that reflect the current state of satisfaction/achievement and the current-foreseeable future state of satisfaction (the number of unachieved TEFs and the number of achieved TEFs as well as the level of their achievement) or the probability of satisfaction/achievement (as a result of implementation of a certain sequence of combinations of interactions of the organism's processes and other processes) of the target evolution functions (blocks of analysis of state and probability of achievement of target evolution functions). Based on this information, the control signals synthesis subsystems has to produce such signals that would lead to satisfaction of the most critical and dissatisfied parameters (in the current, current-foreseeable situation) of the known parameters of the TEFs.

The model described can be implemented both in a firm, unchangeable variant and in a flexible, adaptable variant.

In the first instance (preprogrammed system), the detected sequences of combinations of incoming signals (the detected processes or the groups of processes with certain properties and/or values of characteristics - 4.2.2) that the system can detect and the corresponding to them sequences of combinations of control signals (4.2.3) are firmly predetermined when creating the system. Therefore, the detection and generation of these sequences of signal combinations are performed as a result of interaction 
(both with one another and with other processes of the universe) of the predetermined set of the nervous system's processes that have the predetermined set of properties, values of certain characteristics and the structure of interrelation and interrelationships (3.1, 3.2, 4.1, 4.2).

The advantage of this system is its readiness to work immediately after creation and the absence or the maximal minimization of additional resources consumption for organization of the processes of change of the properties of the nervous system's processes (4.1.2). Its disadvantage is the impossibility to adapt to the changes in the surrounding environment that were not programmed. This disadvantage leads to the necessity of its replacement with the similar one with minor changes (mutations - 3.2). Moreover, only some of these changes will be successful (will contribute to achievement of TEFs of the organism in the changed environment) that will lead to the need in many attempts (of many changed organisms) in order to obtain the required changes. One more disadvantage of such approach is a low complexity of the system (the number of the incoming signals detected by the system and the generation of the outcoming control signals corresponding to them) due to the limited possibility of signals transfer through the hereditary mechanisms (5.3).

In another instance (self-programming/self-adjusting system), the detected sequences of combinations of incoming signals (4.2.2) and the corresponding to them sequences of combinations of control signals (4.2.3) are formed by the nervous system's processes that change their properties and the values of certain characteristics in the process of gaining life experience (4.1.2) depending on what (which incoming influences/signals) the system perceived during its existence and to what extent its reactions (control/outcoming signals) during that time contributed to satisfaction/achievement of its target evolution functions (4.1.3). The better, in a certain situation (certain incoming signals reflecting this situation), the system's reactions (outcoming signals) correspond to satisfaction of its target evolution functions in this situation, the higher the probability of repetition of these actions by the system in the similar situation in the future is (4.1.2).

The advantage of this system is its ability to change (within the capabilities of the system) without the need in its replacement and the ability to gain much more complicated experience with a fewer number and variety of the processes that are required for construction of the nervous system of this type. The disadvantage is the impossibility to ensure achieving TEFs of the organism immediately after the creation due to need in certain time and certain number of attempts for the adaptation of the system to the environment with which the system did not come across earlier or to the changes in a familiar environment.

The most benefits will be received by those living organisms that will have the nervous system combining, to one degree or another, both approaches, i.e. will have the combined system. In order to ensure the functioning of an organism required immediately after the creation and to implement certain functions in its subsequent functioning, use the first approach - preprogrammed blocks (groups) of the nervous system's processes, and in order to ensure the flexible adaptation of the system in a complicated environment, use second approach. Moreover, in such a system, in order to ensure the simultaneous implementation of the preprogrammed functions and their change during the future adaptation and the adaptation flexibility taking into account the preprogrammed functions during the functioning of the organism, it is necessary to implement the ability of interaction (mutual influence, direct or indirect 4.2.1) of the nervous system's processes included to different types of the described blocks (preprogrammed and self-adjusting ones) with each other.

The combined type of the nervous system will be considered hereinafter.

The minimal diagram of such nervous system shall include the following elements (fig. 1):

- receptors (4.2.2) - generate the signals that depend on the availability or the change of the characteristics of any process (and the value of this availability or change) in the surrounding (external with respect to the nervous system) environment within the limits of their sensitivity. The receptors can react to the incoming influences/signals that are the result of the changes in the external environment with respect to the system (organism) or the changes in the system (organism) itself;

- control influences/signals (4.2.3) — signals from the nervous system's processes that can 
influence the properties of other processes of the organism (working organs);

- processing systems (control block) - the set of the processes that, by perceiving the influences from the receptors, create the sequences of combinations of control signals;

- communication systems - the processes that connect (are the intermediates in the signal transfer between the processes) the systems listed above.

The processing systems, in their turn, shall include:

- blocks of preprogrammed reactions - the sets of the processes with the preprogrammed properties and structure of interrelation and interrelationships that ensures the implementation of the programs of analysis of sequences of combinations of incoming signals and synthesis of sequences of combinations of outcoming signals in order to ensure the evolutionarily determined control of the organism's systems;

- blocks of analysis of sequences of combinations of incoming signals (sets of signal detectors) - the sets of the processes that perceive the incoming signals from all receptors of the system and other blocks of the control block and determine the available sequences of combinations of signals (the processes of the universe that are perceived by the nervous system);

- blocks of synthesis of sequences of combinations of outcoming signals (sets of signal generators) - the sets of the processes that perceive data from other blocks of the control block and produce, at the output, those sequences of combinations of outcoming signals that must best of all satisfy the system's target evolution functions in the current and/or future situation;

- blocks of analysis of state and probability of achievement of target evolution functions - the sets of the nervous system's processes that (based on the analysis of incoming signals from the receptors, the blocks of preprogrammed reactions, the blocks of analysis of incoming signals and the blocks of synthesis of outcoming signals) determine to what extent, in a certain point, the target evolution functions are satisfied/achieved or what their probability of satisfaction/achievement (in the future) is as a result of implementation of a certain sequence of combinations of interactions of the processes of the organism and other processes.

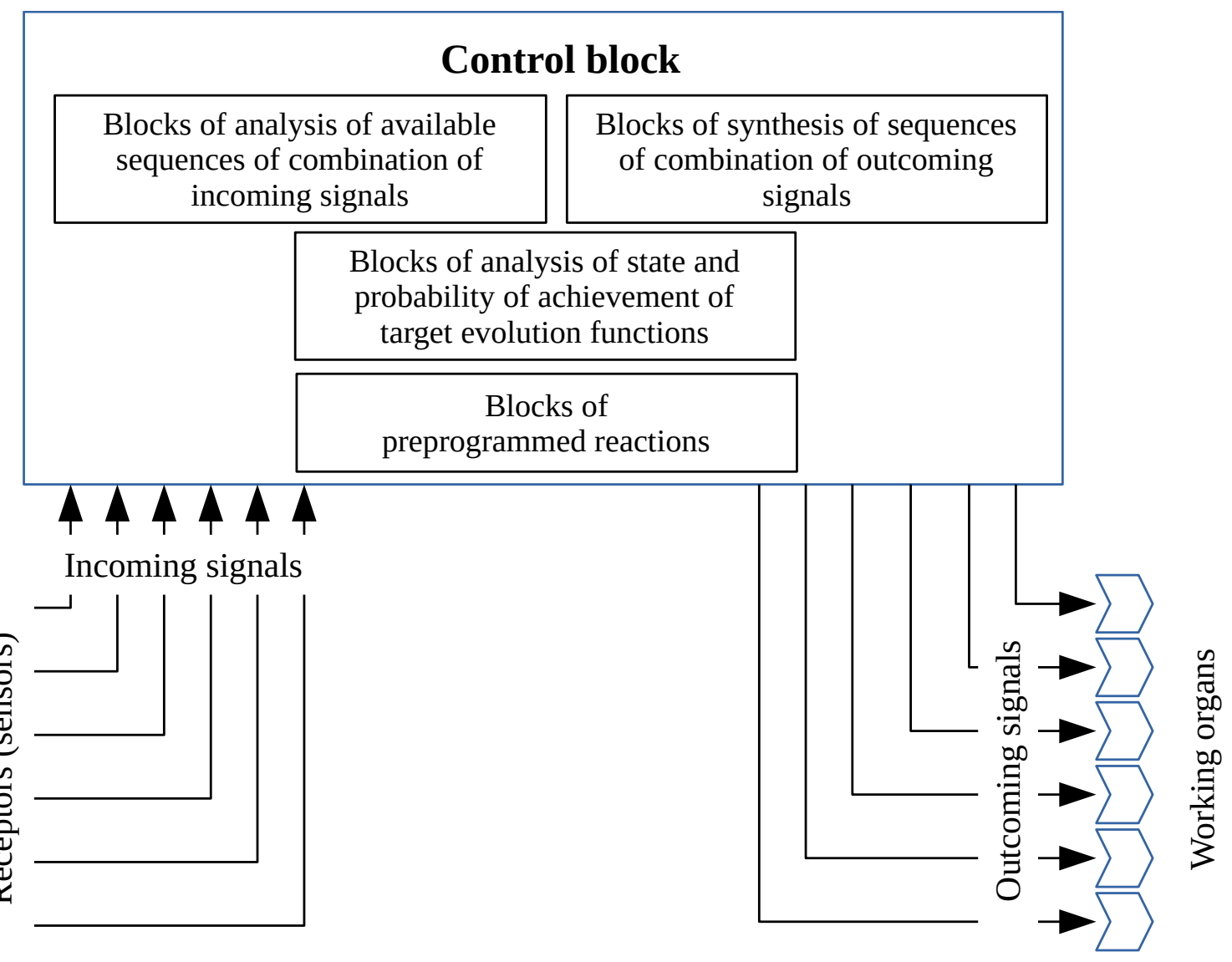

Fig. 1 Block Diagram of Main Elements of Nervous System 


\section{General Structure, Properties, and Principles of Construction of Nervous System}

\subsection{General}

The functioning of the processes of a living organism shall contribute to the efficient and effective achievement of the organism's TEFs (3.2). In order to achieve the organism's TEFs, the nervous system of the organism performs detection and generation of the sequences of signal combinations (4.1, 4.2). Therefore, the detection of a certain sequence of signal combinations is appropriate if it can contribute to achievement of target evolution functions of the organism. Thus, the nervous system of the organism shall have such a structure that every detected sequence of signal combinations influenced, directly or indirectly, the activation of a certain sequence of control signal combinations that will contribute to achievement by the organism of its target evolution functions.

The organism will be better achieve its TEFs (under otherwise equal conditions), that will be able to detect more sequences of combinations (processes of the universe, their characteristics and functions of interdependencies) and generate the sequences of combinations of outcoming signals that will be more appropriate to these situations and possibilities of the efficient and effective achievement (over the short and long term) by the specified organism of its TEFs in such situations $(4.1 .1,4.2)$.

According to the definition of the nervous system, optimal in the context of the achievement of the organism's TEFs interaction of the processes of the organism and the external processes is controlled by the nervous system's processes and is determined by their direct or indirect interaction with the external processes $(4.1,4.2 .1)$. The interaction of the processes of the universe and, therefore, the result of this interaction depend on the properties of the processes, the values of their characteristics as well as the interrelation and interrelationships among the processes (the structure of interrelation/interrelationships for the set of many interacting processes) determined by their corresponding properties (3.1). As the nervous system is the set of interrelated hierarchical processes (4.1.3), its interaction with the external (with respect to the nervous system) processes and, thus, the effectiveness of its management of the organism in order to achieve TEFs of the organism depend on the result of interaction of its processes and, therefore, are determined by the set of these processes, the set of their properties, the values of characteristics and the structure of interrelation and interrelationships of these processes, which is also determined by the values of certain characteristics of the nervous system's processes.

The detected sequences of signal combinations and the generated sequences of signal combinations are reflected in the changes of the properties of the nervous system's processes $(4.1 .3,4.2)$. Therefore, the number of the detected and generated sequences of signal combinations depends on the number of these processes and the variety of their properties. The more complicated the nervous system is (the bigger number of the processes and the groups of the processes, the greater variety of their properties), the better its capabilities will be relating to detection of the current situations (the bigger number of the detected processes, the groups of the processes, their properties and values of characteristics) as well as the more accurate and complicated (thus, efficient and effective for achievement of the target evolution functions) the sequence of combinations of control signals can be generated by the nervous system according to the detected situation.

\subsection{Predetermination of Properties of Nervous System during its Formation}

\subsubsection{General}

The properties of the nervous system (its capabilities relating to interaction with other processes and self-change for better possibilities of achievement of TEFs of the organism in case of such interaction in the future - 4.1.2) of every organism are determined by the set and the properties of its processes (3.1, 
5.1). The current list of the processes of the nervous system and their properties depend on the preliminary list of the processes of the nervous system and their properties as well as on the change of these processes, their properties and values of characteristics that were caused by their interaction both with one another and with other processes $(3.1,4.1 .3)$. Thus, eventually, the current list of the processes of the nervous system, list of their properties, values of characteristics, and the structure of interrelation and interrelationships depend on the initial set of the processes of the nervous system, set of their properties, values of characteristics, the structure of interrelation and interrelationships and on the change of this set as a result of their interaction with one another and with other processes (the life experience of an organism). The initial list of the processes of the organism and their properties (set of the properties, values of characteristics, the structure of interrelation and interrelationships) and, therefore, the initial list of the processes of the nervous system and their properties are defined during the formation (creation) of the organism (in the process of reproduction - 3.2) and, thus, shall be predetermined (in the process of evolution) by the natural selection in the previous generations of the species to which this organism belongs and transferred to it through the hereditary mechanisms (3.2).

\subsubsection{Dependence of Initial Set of Processes of Nervous System of Organism, Their Properties, Values of Characteristics, Structure of Interrelation and Interrelationships on Strategy of Achieving TEFs of Species to which this Organism Belongs}

Every organism develops and exists implementing its TEFs in a certain environment (the set of the processes with which it interacts during its existence). Different organisms can achieve their TEFs in one and the same environment by different ways (implementing different strategies) using different resources of different processes of this environment and, therefore, interacting with different processes of the environment in a different way as well as changing its interaction with these processes in a different way (adapting to the environment). The interaction of the processes of the universe with one another is determined by their properties and the values of characteristics (3.1), which for the living organism depend on the set of its processes, the set of their properties, the values of characteristics and the structure of interrelation and interrelationships of these processes (the subprocesses of the living organism), which, in their turn, depend on the evolutionary path $(3.1,3.2)$ traversed by the species to which this organism belongs. Therefore, the strategy of achieving TEFs for a specific organism is determined by the evolutionary path traversed by the species to which the specified living organism belongs.

The general interaction of the processes of the organism with the processes of the outside environment to ensure achieving TEFs of the organism, i.e. the implementation of the strategy of achieving TEFs, is controlled by the processes of the nervous system (4.1). The interaction of the nervous system's processes with the external (with respect to the nervous system) processes and, thus, the effectiveness of its management of the organism in order to achieve TEFs of the organism depend on the result of the interaction of its processes and are determined by the set of these processes, the set of their properties, the values of characteristics and the structure of interrelation and interrelationships of these processes (5.1). Based on the above in this subsection, it is also fair that the initial set of the nervous system's processes, the set of their properties, the values of certain characteristics and, thus, the structure of interrelation and interrelationships of the processes shall be the ones that ensure the implementation of the strategy of achieving TEFs of the organism in the environment of its existence. And, as specified above (5.2.1), the set of the nervous system's processes, the set of their properties, the values of certain characteristics and, thus, the structure of interrelation and interrelationships of the processes are determined by the evolutionary path traversed by the species to which the living organism belongs and transferred to the specified organism through the hereditary mechanisms.

\subsubsection{Preliminary Determination of Properties of Nervous System's Processes that Determine Rules of Change of These Processes}

During the functioning of the organism with the nervous system of combined type (4.3), the detectors of the sequences of signal combinations (4.3) that detect new/changed processes with which the organism is interacting are formed/change (4.1.2) in it. Also, the set of the corresponding generators (4.3) of the sequences of control signal combinations is formed. All these changes are to take place according to 
certain rules (4.1.2) that allow to ensure the efficient and effective implementation of the organism's TEFs. The interaction and the rules (functions) of interaction of the processes of the universe depend on the set of these processes and their properties (3.1). Therefore, the set of the properties of the processes of the nervous system, including the properties that implement the rules of functioning and the rules of change of the properties of the processes of the nervous system, are to be the ones that ensure these rules (the rules of formation/change of detectors and generators of sequences of signal combinations). As the structure of the nervous system (the set of the processes of the nervous system and their properties) is set during its creation (5.2.1) as to every specimen of a living organism, these rules have to be specified for it beforehand, based on the hereditary information transferred from the previous generations and, thus, determined by the natural selection in the previous generations.

\subsubsection{Predetermination of Blocks of Preprogrammed Reactions}

As specified above (4.3), the blocks of preprogrammed reactions are the sets of the processes with the preprogrammed properties and structure of interrelation and interrelationships that ensure the implementation of the analysis of sequences of combinations of incoming signals and synthesis of sequences of combinations of outcoming signals in order to provide the preprogrammed control of the systems of the organism. These sets of the processes of the nervous system, their properties and the structure of interrelation and interrelationships are formed prior to start of the functioning of the organism (4.3). Therefore, the sets of the nervous system's processes that are included to the specified blocks, the set of their properties, the values of certain characteristics and, thus, the structure of interrelation and interrelationships (both with one another and with other processes of the nervous system and the organism) of these processes are determined by the evolutionary path traversed by the species to which the specified living organism belongs and are transferred to the specified organism through the hereditary mechanisms (5.2.1).

\subsubsection{Predetermination of Detectors of State and Probability of TEF Achievement}

For the survival of the organism it is necessary that all its reactions contribute to achievement of its TEFs as much as possible. As the target evolution functions are aimed at survival of a species of the living organism (3.2) and not only of an individual specimen of a living organism of this species, it is possible to evaluate to what extent these or those reactions of the controlled organism efficiently and effectively contribute to achievement of these TEFs only over the long term, i.e. only on the basis of the fact of survival of the species represented by this specimen of the organism in the environment of existence and evolution of this species. Thus, the system that is responsible for the evaluation of the state and probability of TEF achievement (determines the processes that reflect known to the system parameters of the target evolution functions that are dissatisfied/satisfied in the current situation or can be, with a certain probability, dissatisfied/satisfied in the foreseeable future situation and to what extent - 4.1, 4.3) - the set of the detectors of state and probability of TEF achievement - have to be based on the hereditary information transferred from generation to generation. Therefore, the set of the detectors of state and probability of TEF achievement have to either be composed of the detectors the detecting process in which is specified beforehand (in the previous generations), and their set, the set of their properties, the values of characteristics and the structure of interrelation (required to ensure the detection of these processes - 5.2.2) were transferred through the hereditary mechanisms (5.2.1) — predetermined detectors of state and probability of TEF achievement - or include (in the nervous system of combined type - 4.3) such detectors as the basis, and the processes that are detected by other (those that are not preprogrammed - self-adjusting) detectors of state and probability of TEF achievement have to be determined depending on the processes detected by the preprogrammed detectors of the TEF achievement. In this case to increase the probability and the value of satisfaction of the organism's TEFs, the self-adjusting detectors of state and probability of TEF achievement have to change according to such rules that will allow them to adjust themselves to the detection of such processes during the implementation of which the probability of implementation of the processes that are detected by the preprogrammed detectors of the state and probability of TEF achievement in case of increase of the state and probability of achievement of TEFs, is increased. I.e. the self-adjusting detectors of state and 
probability of TEF achievement have to adjust themselves to the detection of the processes interrelated with the processes that are detected by the preprogrammed detectors of the state and probability of TEF achievement in case of increase of the state and probability of achievement of TEFs and, therefore, these processes are a part of a certain hierarchically higher process (3.1) whose implementation results in the satisfaction of the organism's TEFs.

The preprogrammed detectors of the state and probability of TEF achievement have to be active only during the period of development of the organism when it is reasonable for achievement of the corresponding TEFs and with such a value of influence on other processes with which the implementation of the corresponding TEFs with regard to other organism's TEFs is reasonable. The time of activation and the value of influence (on other processes of the universe) of every block of preprogrammed TEFs detectors are also determined based on the fact of survival of the species represented by this organism and, thus, have to be transferred through the hereditary mechanisms (5.2.1).

\subsubsection{Predetermined Determination of Adjustment of Self-adjusting Processes of Nervous System to Interaction with Processes of the Universe under Influence of Signals from Blocks of Analysis of State and Probability of TEF Achievement and Preprogrammed Systems}

In the nervous system of combined type the self-adjusting processes of the nervous system adjust themselves to the interaction with other processes of the universe in the process of interaction with these processes (4.3) while also receiving the influences from the processes of the nervous system included to the blocks of analysis of state and probability of TEF achievement and preprogrammed systems (4.3). Moreover, this interaction itself also takes place under the influence of the preprogrammed systems (4.3). Thus, the adjustment of the self-adjusting processes of the nervous system to such interaction with the processes of the universe that will lead to the implementation of the strategy of achieving TEFs depends on these systems. Therefore, the set of the processes of the nervous system included to the blocks of analysis of state and probability of TEF achievement and preprogrammed systems, the set of their properties, the values of certain characteristics and the structure of interrelation and interrelationships (both with one another and with the self-adjusting processes of the nervous system) are to be the ones that ensure the adjustment of the self-adjusting processes to such interaction with the processes of the universe that will lead to the implementation of the strategy of achieving TEFs of the organism in the environment of its existence (5.2.2). The set of the specified processes, the set of their properties, the values of characteristics and the structure of interrelation and interrelationships are determined by the evolutionary path traversed by the species to which the specified living organism belongs and transferred to the specified organism through the hereditary mechanisms $(5.2 .1,5.2 .4,5.2 .5)$.

\subsubsection{Substrategy of Strategy of Achieving TEFs of Organism}

Different organisms can achieve their TEFs in one and the same environment by different ways (implementing different strategies) using different resources of different processes of this environment and, therefore, interacting with different processes of the environment in a different way as well as changing its interaction with these processes in a different way (5.2.2). The hierarchically higher (from the initial level) processes in the universe are mainly similar processes with a different level of similarity (3.1). Therefore, in the environment of the organism's existence there can be groups of similar processes, the similarity of which to each other is greater than their similarity with the processes that belong to other such groups. The processes of the universe belonging to each of these groups can in different ways influence the achievement of TEFs by this organism. Consequently, the nervous system of the organism can use different strategies (substrategies of the overall strategy) to achieve TEFs when interacting with the processes of the universe, belonging to each of these groups.

The strategy of achieving TEFs for a specific organism is determined by the evolutionary path traversed by the species to which the specified living organism belongs (5.2.2). Therefore, described substrategies of achieving TEFs for a specific organism is determined by the evolutionary path traversed by the species to which the specified living organism belongs. 


\subsection{Limitation of Resources - Limitation of Capabilities and Optimization of Nervous System}

\subsubsection{Limitation of Sets of Detected and Generated Signals as a Result of Limitation of Resources}

As a result of the limitation of the resources of the organism (3.2) that it can provide for the functioning of the nervous system, the nervous system has a limited complexity (the number of the processes and their groups and the variety of the set of the properties of these processes). Therefore (5.1), at any time of its existence, the system has the limited (finite) set of the sequences of signal combinations that it is able to detect and the limited set of the sequences of control signal combinations corresponding to it that it is able to generate. With such limitations, the nervous system can ensure the detection of the sequences of signal combinations and the generation of the sequences of control signal combinations required for achievement of TEFs of the organism based on the selection of the sequences of signal combinations that are the most relevant for the current situation from its current limited sets. I.e., at a certain point of time, the nervous system activates the most the sequence of control signal combinations (from the finite set) that is connected with the detected combination (from the finite set) that is the most relevant for the current incoming sequence of signal combinations. Moreover, the activated sequence of control signal combinations shall, as often as possible and with the highest level of satisfaction, lead in the past to achievement of TEFs of the organism at this detected incoming sequence of signal combinations (4.1).

\subsubsection{Limitation of Capabilities when Transferring Hereditary Information - Unification of Properties of Processes of Nervous System and Formation of Structure of Nervous System According to Certain Rules}

The set of the processes of the nervous system of a certain organism, the set of their properties, the values of certain characteristics and, thus, the structure of interrelation and interrelationships of the processes are determined by the evolutionary path traversed by the species to which the living organism belongs, whose part this nervous system is, and transferred to the specified organism through the hereditary mechanisms (5.2.2). As the mechanisms of hereditary transmission of information have, as other systems of the organism (3.2), certain limitations determined by the number and the properties of the processes that implement them, thus, the complexity of the organism's nervous system, which is formed on the basis of this limited hereditary information, is also limited (the list of processes of the nervous system and the variety of their properties are determined by the list of processes of the hereditary mechanism and the variety of their properties and values of characteristics - 3.2, 3.1). The capabilities of the nervous system, when ensuring achievement of the TEFs, as specified above (5.1), depend on the complexity of the system. Therefore, taking into account the limitation of the hereditary mechanism, the organism will have the advantage the formation of the nervous system of which (based of the limited volume of hereditary information) will lead to a more complicated nervous system (bigger number of the nervous system's processes, bigger set of their properties and greater complexity of the structure of their interrelation and interrelationships). The increase in complexity of the nervous system in case of the limited volume of hereditary information is possible thanks to use of the same hereditary information blocks for formation of different nervous system's processes that implement the same or the similar functions in the nervous system, i.e. in case of unification of the processes of the nervous system at different levels. In such a case, the processes that implement the same functions of the nervous system shall have the maximally simple identical (uniform) set of properties required for the implementation of their functions in the nervous system with the minimally possible directly transferred through the hereditary mechanism information about the differences of various groups of these processes of the nervous system. And the structure of interrelation and interrelationships of these processes of the nervous system shall be formed on the basis of the maximally simple uniform rules that will be implemented thanks to the uniform properties of these processes with the minimally possible directly transferred through the hereditary mechanism information about this structure of interrelation and interrelationships. 


\subsubsection{Minimization of Resources Consumption by Means of Optimization of Properties (Set of Subprocesses and Their Properties) of Processes of Nervous System}

The organism whose nervous system will consume fewer resources when functioning under otherwise equal conditions (the set of the processes detected by the nervous system and the set of the corresponding generated control signals) will achieve the target evolution functions more efficiently. As the detected sequences of signal combinations and the generated sequences of signal combinations are reflected in the changes of the properties of the processes of the nervous system (4.1.2, 4.1.3), the nervous system whose properties of processes will be the ones that will ensure fewer consumption of the resources for the change of the properties of these processes (in the first place, for the change of those properties on which the signal detection and generation directly depend) will be more efficient. Besides, the properties of these processes and the implemented changes of their properties have to be the ones that ensure the minimal utilization of the resources when performing the functions (signal detection and generation) of these processes of the nervous system.

\subsubsection{Limitation and Evolutionary Determination of Set of Processes (and Their Properties) with which Nervous System of Organism can Interact during the Life Path of this Organism}

The nervous system of the organism has a limited complexity (the number of the processes and their groups, the variety of the set of the properties of the nervous system's processes and the structure of their interrelation and interrelationships) and, thus (5.3.1), it has a limited (finite) set of the sequences of signal combinations (processes and their properties - 4.2.2) that it is able to detect and a limited set of the sequences of control signal combinations (processes and their properties - 4.2.3) that it can influence. The interaction of the processes of the universe is determined by the set of their properties, the values of characteristics and, thus, the structure of their interrelation and interrelationships (3.1). The set of the processes of the nervous system of a certain organism, the set of their properties, the values of certain characteristics and, thus, the structure of their interrelation and interrelationships are determined by the evolutionary path traversed by the species to which the living organism belongs, whose part this nervous system is, and transferred to the specified organism through the hereditary mechanisms $(5.2 .1,5.2 .2)$. Therefore, the set of the processes of the universe (and their properties) with which the nervous system's processes can interact is determined by the evolutionary path traversed by the species to which the living organism belongs, whose part this nervous system is, in the environment of existence of this species (5.2.2).

\subsubsection{Limitation and Optimization of Number of Processes with which Nervous System Interacts Simultaneously}

The limitation of the complexity of the nervous system (5.3.1) leads to the limitation of the number of the processes and their properties with which, in a certain situation (4.1.2), the nervous system's processes can simultaneously interact $(3.1,4.2)$ (the limited, finite set of the processes and their properties that the nervous system's processes are able to detect simultaneously and the limited set of the processes and their properties that they can influence simultaneously). The interaction of the nervous system's processes with one another and with other processes of the universe has to lead to the maximally efficient and effective (4.1.2) achievement of TEFs of the organism (4.1.3). Thus, the set of the nervous system's processes, the set of their properties and the structure of their interrelation have to be the ones that, in a certain situation, ensure the ability of detection of such minimally required set of the processes and generation of the corresponding minimally required set of influences in order for the interaction of the processes of the universe implemented as a result of this to lead to the maximally efficient and effective achievement of TEFs of the organism in this situation (4.1). These minimally required sets of simultaneously detected processes and their properties and the sets of the influences simultaneously generated by the processes of the nervous system are determined by the strategy of achieving TEFs implemented by the organism (5.2.2) and the efficiency and effectiveness of achieving TEFs of the organism during the previous interactions of the processes of the organism with other processes of the universe similar to those with which the current interaction takes place in the situations similar to the current one (4.1). 
5.3.6. Minimization of Resources Consumption by Means of Optimization of Functioning of Processes of Nervous System in Order to Ensure Certain Level of TEF Achievement (Satisfaction)

The organism whose nervous system will consume fewer resources when functioning under otherwise equal conditions (the set of the processes detected by the nervous system and the set of the corresponding generated control signals) will achieve the target evolution functions more efficiently. The processes of the nervous system function in order to ensure achievement of TEFs of the organism (4.1). At any time, the level of achievement (satisfaction) of various TEFs of the organism is detected by the blocks of analysis of state and probability of achievement of target evolution functions (4.3) and can be different (from complete dissatisfaction — zero satisfaction — to complete satisfaction). At the same time, the processes of the organism's nervous system interact only with the limited set of the processes of the universe (5.3.5). Therefore, to minimize the utilization of the resources, at the same time, only those nervous system's processes that are required to control the level of TEF achievement and the processes that are required to ensure the detection and the generation of the sequences of combinations of signals that will allow to achieve the required level of achievement of TEFs of the organism have to function (detect/generate the sequences of signal combinations). Thus, the set of the nervous system's processes, their properties and the structure of interrelation and interrelationships have to be the ones that ensure such selective functioning of the nervous system's processes. The set itself of the nervous system's processes, their properties and the structure of interrelation and interrelationships are predetermined in the previous generations of the species of the living organism to which this nervous system belongs and are transferred to it through the hereditary mechanisms (5.2.1).

\subsection{Optimization of Structure of Interrelationships and Properties of Processes of Nervous System Based on Regularities in Structure of Interrelationships and Properties of Processes of the Universe}

\subsubsection{Correlation between Structure of Interrelationships of Processes of Nervous System and Structure of Interrelationships among Processes of the Universe and Their Properties}

The processes of the universe interact with one another creating the hierarchically higher processes according to certain rules determined by their properties (3.1). The interaction of the processes of the universe according to these rules results in the ability of creation the hierarchically higher processes only with a certain (the one that can emerge according to the specified rules) structure of interrelation of their subprocesses and, thus, interdependence of their properties and the values of characteristics.

The nervous system of an organism is a hierarchical process that is a set of the interrelated hierarchical processes interacting with one another and perceiving the influences from/influencing the external (with respect to the processes of the nervous system) processes (4.1.3). Moreover, the different processes of the nervous system can detect or influence the different processes of the universe or their certain properties and/or subprocesses. The processes of the nervous system themselves have a certain structure of interrelation (within the hierarchically higher processes that they form - the nervous system or its substructures) that determines which of these processes and in which order can interact with one another (3.1, 4.1.3). Therefore, the processes of the nervous system can detect/generate the influences from/on certain processes of the universe or their properties perceiving/exerting the influences from/on them indirectly (4.2.1) through other nervous system's processes that, in their turn, can detect/generate the influences perceiving/exerting the influences from/on certain properties or subprocesses (and/or their properties or subprocesses) of these processes of the universe.

The processes of the nervous system detect/generate the influences from/on certain processes of the universe or their certain properties (the values of certain characteristics of the processes) perceiving/exerting the influences in the form of the interrelated sequences of combinations of influences (4.2). Moreover, the order of change of the properties of the nervous system's processes and their interdependence reflects (is similar) the order of change of the corresponding properties of the processes of the universe and their interdependence (4.2). Thus, to ensure the detection or generation of the 
interrelated sequences of combinations of influences from/on the processes of the universe by the nervous system's processes that receive/exert the influences from/on these processes of the universe indirectly through other processes of the nervous system, the order of interaction (interdependence) of the nervous system's processes that detect the interdependence of the interrelated properties and/or the interaction of these interrelated processes of the universe or generate the influences on them has to reproduce (be similar) the order of interdependence of the specified processes of the universe or their properties (3.1, 4.2). Therefore, the structure of interrelation of these processes of the nervous system has to reproduce (be similar) the structure of interrelation of these processes of the universe or their properties (3.1).

In the nervous system of combined type (4.3) for the nervous system's processes that detect or generate the influences from/on the processes of the universe and are included to the blocks of preprogrammed reactions, it is predetermined which processes or their properties will be detected by them and which influences will be exerted. Therefore, it is predetermined which way they have to interact with one another and with other processes and their interrelation is set in the form of the interrelationships the structure of which is completely determined by the hereditary mechanisms (5.2.4).

As for the nervous system's processes that are not included to the blocks of preprogrammed reactions, the detected sequences of combinations of incoming signals and the corresponding to them sequences of combinations of control signals, which match the processes of the universe and their properties (4.2.2, 4.2.3), are formed in the process of gaining life experience (through the changes of the properties of the nervous system's processes) depending on what (which incoming signals) the system perceived during its existence and to what extent its reactions (outcoming - control signals) during that time contributed to the satisfaction of its target evolution functions $(4.3,4.1)$. I.e., as for these processes of the nervous system, the processes of the universe or their properties that will direct detect or that these processes of the nervous system will influence and their interrelation are not predetermined. However, taking into account the limitation of the capabilities of the nervous system, the set of the processes of the universe that can determine and that the nervous system's processes can influence are limited and depend on the strategy of achieving TEFs of the organism in the environment of its existence (5.3.4). I.e., as for these nervous system's processes, there is a predetermined set of the processes of the universe to the detection and generation of influences from/on which these nervous system's processes shall adjust. As specified above, the processes of the universe interact according to certain rules, which lead to certain, allowed by these rules, structure of their interrelation. Thus, the allowable structure of interrelation of the groups of the processes that can determine and that the nervous system's processes can influence is also limited by the structure of interrelation of the groups of the processes with which the organism can interacts when implementing its strategy of achieving TEFs in the environment of existence.

Taking into account that the nervous system has limited capabilities (5.3) and that the organism have the advantage whose processes of the nervous system consume fewer resources for implementation of its functions (3.2), the nervous system will be more efficient whose processes will consume fewer resources for adjustment to detection or generation of the influences from/on certain groups of the processes of the universe and, therefore, on formation of the required for this interrelation and interrelationships with one another. As specified above, when detecting certain processes of the universe, the order of interaction and the structure of interrelation of the nervous system's processes that detect the interdependence of the interrelated properties and/or interaction of the interrelated processes of the universe or generate the influences on them are to reproduce the order of interaction and the structure of interrelation of these processes of the universe or their properties. Therefore, the more the initial structure of interrelation of the nervous system's processes that adjust themselves to the detection or generation of the influences from/on certain groups of the processes of the universe is similar to the allowable structures of interrelation of these processes of the universe, hence, the less changes will be required to be implemented and the fewer resources will have to be consumed by the processes of the nervous system for formation of the required structure of interrelation and interrelationships with one another in order to detect these processes and their properties and interrelation among them. I.e. the initial structure of interrelation of the processes of the nervous system has to correspond to the possible structures of interrelation of the processes of the universe with which these processes of the nervous system can interact when implementing the strategy of achieving TEFs of the organism.

The structure of interrelation and interrelationships of the nervous system's processes is determined by 
the evolutionary path traversed by the species to which the living organism belongs, whose part this nervous system is, and transferred to it through the hereditary mechanisms (5.2).

\subsubsection{Hierarchy and Complexity of Structure of Detectors and Generators of Sequences of Signal Combinations}

According to the initial assumption (3.1), the universe is a set of hierarchical processes, and the processes in the universe, including a living organism itself, are a certain combination of other, hierarchically lower processes (except for the processes of the initial level of the hierarchy). The processes of the universe change and interact with one another according to the unchangeable rules determined by their properties and the values of characteristics (3.1). During the interaction with different processes, the nervous system of the organism, therefore, perceives these processes as the set of the sequences of combinations of signals from its receptors and influences these processes generating the sequences of combinations of outcoming signals (4.2.2, 4.2.3). Moreover, these sequences of combinations of incoming signals and the sequences of combinations of outcoming signals generated by the nervous system will reflect the hierarchy of the processes of the universe, the hierarchy of their properties, the rules of interaction between the processes (both of single and different levels of hierarchy) and the values of characteristics (4.2.1). I.e., it is possible to identify the levels of the signal hierarchy in every sequence of combinations of incoming/outcoming signals of the nervous system. Moreover, every level of the hierarchy of the signals of the nervous system will reflect the corresponding level of the hierarchy of the processes of the universe, and more complicated processes, their properties and causeand-effect relations, therefore, can be reflected as the sequence of combinations of sequences of combinations of signals from/to the lower-level processes.

The organism whose nervous system will consume fewer resources when functioning (3.2) under otherwise equal conditions (the set of the processes detected by the nervous system and the set of the corresponding generated sequences of combinations of control signals) will achieve the target evolution functions more efficiently. Taking into account the limitation of the capabilities of the nervous system (5.3) and the hierarchy of the combination of sequences of incoming signals received by it and the generated outcoming signals, the nervous system, under such conditions, will be more efficient in which this hierarchy will be taken into account. I.e., to avoid duplication of detection/generation of one and the same sequences of combinations of signals from/to the processes (or their properties) of the lower level of the hierarchy, for different processes (or their properties) of the higher level of the hierarchy, the systems of detection and generation of the signals in the nervous system shall have the hierarchical structure. At each level of such structure the sets of the combinations of signal sequences that correspond to a certain set of processes (properties of processes and values of their characteristics) of a certain level of the hierarchy shall be identified/generated. The sets of the combinations of signal sequences obtained at certain level can be processed at the next level of the structure of the nervous system as individual components of the processes (and/or their properties) of the higher level of the hierarchy. To obtain the combinations of signal sequences that will correspond to the next level of the hierarchy of the processes (the properties of the processes and the values of characteristics) of the universe and, therefore, will be the combination of combinations of signal sequences from the previous levels of the structure of the nervous system, the interrelation and interrelationships between the corresponding hierarchical blocks of the nervous system shall correlate to the hierarchy of the interrelation and interrelationships (inclusion) of the processes (the properties of the processes and the values of characteristics) of the universe (5.4.1).

The optimization of the structure of these hierarchical blocks of the nervous system (the number of the blocks and their interrelationships) shall be the one to ensure the detection/generation of the sequences of combinations of incoming/outcoming signals for those processes of the universe (the properties of the processes and the values of characteristics) that are important for the organism, to which this nervous system belongs, to achieve its TEFs in the environment of its existence (the set of the hierarchical processes of the universe with which this living organism interacts in the process of its activity) according to its strategy of achieving TEFs. Therefore, the structure of these hierarchical blocks, as specified above (5.2), shall be predetermined in the previous generations of the species to which this living organism belongs. 


\subsubsection{Parallelism of Structure of Blocks of Analysis/Synthesis of Sequences of Combinations of Signals}

For the efficient and effective achievement by the organism of its target evolution functions, the speed of the organism's reactions is important. Not only the speed of response of individual elements of the nervous system is important for providing the required speed of the signal detection and generation, but the approach to its structure in the whole as well. More effective, in the context of speed of signal processing, will be the nervous system that will perform the simultaneous processing (signal detection or generation) of the maximally bigger array of sequences of signal combinations available for processing. This approach can be provided (under otherwise equal conditions) by parallel processing of the specified array of sequences of signal combinations. Thus, the structure of the blocks of analysis/synthesis of sequences of combinations of signals shall have the working in parallel elements (the set of the nervous system's processes) for the implementation of the simultaneous processing of available sequences of signal combinations.

For the achievement of the target evolution functions, the actual system shall use the minimum of organism's resources at the acceptable speed, i.e. effectively combine the hierarchy and the parallelism in its structure.

\subsubsection{Parallelism of Structure of Blocks of Detectors and Generators of Sequences of Combinations of Signals at Every Level of Hierarchy}

According to the initial assumption, the universe is a set of hierarchical processes (3.1). Moreover, the existence of a certain set of similar processes is possible at every level of the hierarchy of the processes of the universe. The processes of the nervous system have, in their turn, the hierarchical structure and at each level of the hierarchy are identified/generated the sets of combinations of signal sequences that match a certain set of the processes of the universe, a certain level of hierarchy (5.4.2). Moreover, for the optimization of the speed of processing (detection or generation) of the sets of the signal combinations shall be used the working in parallel blocks that will process these signals (5.4.3). Thus, every level of the hierarchy of detectors and generators of sequences of signal combinations shall be composed of the working in parallel detectors/generators (the sets of the nervous system's processes) that will ensure the parallelism of processing of the sets of the sequences of signal combinations received by them at this level of the hierarchy.

\subsubsection{Specialization and Localization of Receptors and Outcoming Signals Depending on Type of Interaction with External Processes}

Every process has a certain set of properties (characteristics and functions of their interdependence), which manifest themselves (for other processes) thanks to the interaction of this process with the specified processes $(3.1,4.2)$. Different properties of the processes cause and determine different types of interaction of the processes (3.1). Taking into account the limitation of the resources of the nervous system, the organism will achieve its TEFs better, whose sensitivity to a certain type of interaction (influence caused and determined by the property - characteristic and functions of its dependence on other characteristics of a certain type in the process being detected) or possibility of a certain type of interaction (influence in order to change the property [characteristic] of a certain type in the process on which the influence is exerted) and location (interrelation with other processes of the organism) of receptors (4.2.2) and nervous system's processes that produce the outcoming signals will be optimized according to the probability of their interaction with a certain process exactly in this area of the organism and the criticality (for implementation of the strategy of achieving TEFs of the organism) of detection of certain properties of this process or influence on them in a certain area of the organism.

\subsubsection{Determination of Structure of Nervous System by Interaction with Processes of the Universe through Sets of Their Properties}

Taking into account the specialization and localization of receptors and generators of outcoming signals (5.4.5) and the structure of their interrelation (5.4.1), various groups of interrelated receptors or 
generators of outcoming signals interact with the processes of the universe using a certain property (characteristic and functions of its dependence on other characteristics) of these processes that cause and determine the possibility of their interaction with this type of receptors (the nervous system's processes 4.2.2) or generators of outcoming influences (4.2.3). Therefore, as every such group of the processes of the nervous system, other nervous system's processes that interrelated with these groups and indirectly (4.2.1), through this groups, interact with the certain processes of the universe (detect and generate the influencing from/on it sequences of signal combinations perceiving or exerting the influences from/on other processes of the nervous system - 4.2) implement the interaction with it thanks to the corresponding property (the one through which the receptors or generators of outcoming signals with which they are interrelated interact with these processes of the universe) of this process of the universe. As the processes of the universe have a certain set of properties (3.1), the living organism can have several groups of interrelated receptors or generators of outcoming signals (depending on the strategy of achieving TEFs 5.2.2) each of which can interact with the processes of the universe thanks to the property (characteristic) that is different from those thanks to which other groups of the processes of the nervous system interact with these processes of the universe. Thus, the processes of the nervous system interact (detect and generate the influencing sequences of signal combinations) with every process of the universe by detecting it as / influencing it through the caused and determined by a certain type of interaction, due to a certain property (characteristic with the corresponding function of its dependence on other characteristics) of this process of the universe, parallel sets of hierarchical (5.4.2) sequences of signal combinations. Each of the specified groups of the nervous system's processes that processes certain specified hierarchical sequences of signal combinations shall, therefore, have its hierarchical structure (5.4.2). The types of the properties (characteristics and functions of their dependence on other characteristics) of the processes of the universe that are used by the nervous system's processes for interaction with these processes are conditioned by the strategy of achieving TEFs by an organism, to which this nervous system belongs, are determined by the set of the nervous system's processes, the set of their properties, the values of certain characteristics and the structure of their interrelation and interrelationships, which are determined by the evolutionary path traversed by the species, to which this living organism belongs, and transferred to the specified organism through the hereditary mechanisms (5.2).

\subsubsection{Interrelation in Structure of Parallel Hierarchical Blocks of Detectors and Generators of Sequences of Combinations of Signals as a Result of Interrelation of Different Hierarchical Properties of Processes of the Universe Thanks to which They Interact with Nervous System's Processes}

The processes of the universe are the interrelated set of properties (3.1). The processes of the nervous system interact with the processes of the universe receiving from them / influencing them with the interrelated sequences of signal combinations (4.2). The perception and implementation of influences and processing of groups of sets of sequences of signal combinations, which are caused and determined by different types of interaction with the processes of the universe, which are realized thanks to different properties (characteristics and functions of their dependencies on other characteristics) of these processes, are implemented by the parallel hierarchical groups of processes of the nervous system $(5.4 .3,5.4 .6)$.

Taking into account the correlation between the structure of interrelation of the nervous system processes and the structure of interrelation of the processes of the universe and the structure of interrelation of their properties (5.4.1), the groups of blocks of signal detection and generation in the nervous system that process the sequences of signal combinations, which are caused and determined by different types of interaction with the processes of the universe, shall have the interrelated structure that will reflect the interrelation of certain hierarchical properties (characteristics) of the hierarchical processes with which the interaction is carried out. The optimization of the number and the structure of interrelation of these blocks of the nervous system shall be the one to ensure the detection/generation of the sequences of combinations of incoming/outcoming signals for those processes (the properties of the processes and the values of characteristics) that are important for the organism (to which this nervous system belongs) to achieve its TEFs in the environment of its existence (the set of the hierarchical processes of the universe with which this living organism interacts in the process of its activity) according to its strategy of achieving TEFs(5.2.2). Therefore, the structure of interrelation of the specified blocks, as specified above 
(5.2), shall be predetermined in the previous generations of the species to which this living organism belongs.

\subsubsection{Optimization of Number of Detectors and Generators of Sequences of Signal Combinations at Every Level of Hierarchy}

Every level of the hierarchy of the blocks of analysis/synthesis of sequences of combinations of signals shall be composed of the working in parallel sets of the nervous system's processes that detect/generate the sequences of combinations of signals (5.4.4). The number of these nervous system's processes at every level of the hierarchy determined the number of the processes of the universe of a certain level of the hierarchy that can be detected/generated by the processes of the nervous system (5.3.1, 5.3.4, 5.3.5, 5.4.2).

The maximal number of the detected processes of the universe of a certain level of the hierarchy is limited by the number of these processes in the environment of existence where the species was developing and the living organism that belongs to this species implements the strategy of achieving TEFs. In different situations, each of these processes can differently interact with this living organism and, thus, in case of interaction with a certain group of receptors, which perceive a certain type of influences (5.4.5), can influence different random subgroups of this group of receptors. Taking into account the functions and the limitation of the capabilities of the living organism's nervous system, the number of the nervous system's processes that detect the signals at every level of the hierarchy and the character of their interrelationships with the nervous system's processes from which they get influences shall be the ones that can ensure the detection of the processes of the universe of a certain level of the hierarchy with such an accuracy of determination (localization) of the subgroup of the processes that detect the influences from this process of the universe at the preceding level of the hierarchy, starting from the detecting receptors, and with such an accuracy of determination of similarity of the detected processes that is required to ensure the implementation of the strategy of achieving TEFs by the organism to which this nervous system belongs. The number of the detectors/generators in each of the hierarchical blocks, as specified above (5.2), shall be predetermined in the previous generations of the species to which this living organism belongs.

Interrelationships between blocks of

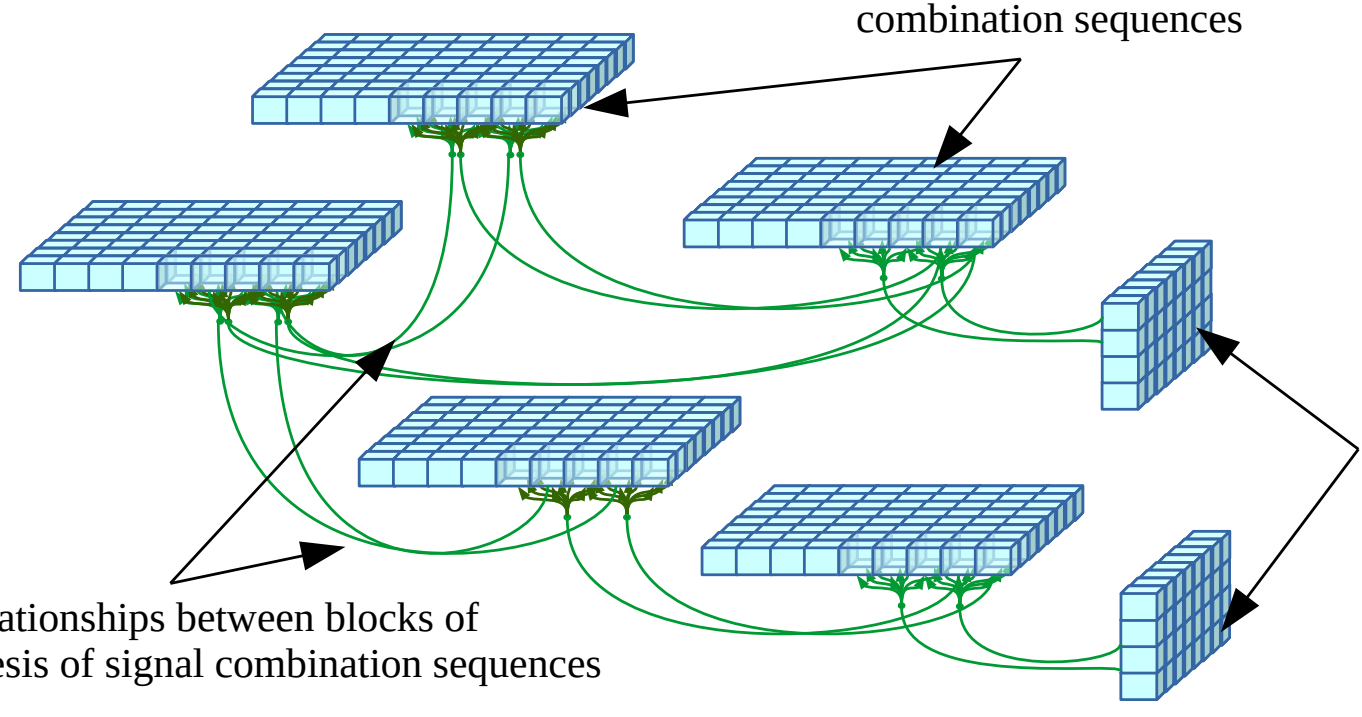
analysis/synthesis of signal combination sequences

Fig. 2 Structural Hierarchy of Blocks of Analysis/Synthesis of Signal Combination Sequences 


\section{Minimal Uniform Process of Nervous System (Structure and Rules of Formation and Functioning Based on Regularities in Interaction of Processes of the Universe)}

\subsection{General}

\subsubsection{Similarity of Key Functions of All Processes of Nervous System}

The processes of the nervous system are interrelated processes that interact with one another in order to implement the function of the nervous system (4.1.3). When interacting, each process of the nervous system perceives influences (signals) from other processes and generated the influences (signals) on other processes (4.2). The influences generated by the nervous system on the external, with respect to the nervous system, processes (outputs of the nervous system) depend on the current-foreseeable situation and the probability of efficient and effective achievement of TEFs in the current (current-foreseeable) situation (4.1.1, 4.1.2). The current situation (the set of interrelated processes of the universe, their properties and the values of characteristics - 4.1.2) is detected by the nervous system's processes that directly or indirectly perceive influences from the external (with respect to the nervous system) processes (4.2, 4.3, 5.4). The foreseeable situation and the probability of efficient and effective achievement of TEFs in a certain current-foreseeable situation are determined based on the changes implemented in the processes of the nervous system during previous interactions (perception of influences and generation of influences) with the external processes with successful or unsuccessful achievement of TEFs of the organism (4.1). Therefore, taking into account the interrelation of all processes of the nervous system and their interaction when generating the outcoming signals, in order to ensure the dependence of the influences generated by the nervous system on the external (with respect to the nervous system) processes (outputs of the nervous system) from the current-foreseeable situation and the probability of efficient and effective achievement of TEFs in this (current-foreseeable) situation $(4.1 .1,4.1 .2)$ it is necessary that the generated by all processes of the nervous system influences (signals) depend on the current situation detected by these processes, and / or on the current properties (3.1) and values of characteristics of the nervous system's processes, which, in their turn, are determined by the previous interactions and initial, predetermined properties and values of characteristics $(5.2 .1,5.2 .2,5.2 .3)$ of the nervous system's processes. Thus, in view of the above each process of the nervous system shall detect a certain process that is part of the current-foreseeable situation or was part of a certain current-foreseeable situation in previous interactions. As the processes of the universe are detected by the processes of the nervous system in the form of the interrelated sequences of signal combinations (4.2.2), the generation of a signal by every process of the nervous system shall be carried out depending on certain detected interrelated sequences of signal combinations (or one sequence of signals) that influence this process. I.e. every process of the nervous system, by perceiving the signals (influences) from other processes, shall carry out the detection of certain interrelated sequences of signal combinations that correspond to a certain process or its property and generate, at this detection, the signals (influences) on other, following, processes (external or internal with respect to the nervous system).

Hereinafter, when using the term "detection of signal combinations", it will mean corresponding to this process the generation of the sequence or sequences of combinations of signals.

The organism and its nervous system, in each specific situation, interact not with all processes and in all its possible states (the values of characteristics), with which they can interact, but with a limited set of the processes (only with those with which there is a corresponding interrelation -3.1, 4.2.1). Therefore, in each situation, only some processes of the nervous system will operate (detect the current situation and generate signals).

As mentioned above, the detection of the interrelated sequences of signal combinations and the generation, at this detection, of the outcoming sequences of signal combinations or at least of one signal sequence (complicated and long-lasting sequences of combinations of outcoming signals can be formed 
by the combination of the sequences of outcoming signals of several such processes that will generate the signals) is performed by every process of the nervous system. Therefore, the changes of the properties of the nervous system's processes connected with the detection of new processes or generation of new outcoming signals shall take place in every process of the nervous system. However, as not all processes of the nervous system come into action (are involved) in a certain current situation, these changes in this situation take place only with nervous system's processes that directly or indirectly perceive and generate the influences (signals) during interaction of the nervous system with the new external processes in the current situation as well as with the nervous system's processes that are interrelated with these coming into action processes of the nervous system (3.1, 4.2.1).

\subsubsection{Minimal Uniform Process as Basis of All Processes of Nervous System}

As specified above (5.3.2), the processes that implement identical functions of the nervous system shall have as much as possible identical (uniform) structure (the set, the properties as well as the interrelation and interrelationships of their subprocesses) and the set of the properties required for implementation of their functions in the nervous system. Besides, all processes of the nervous system implement identical functions (6.1.1) - detect the interrelated sequences of signal combinations and generate at least one outcoming signal sequence and change, at these detection and generation, according to certain rules. Thus, taking into account the specified above statements, all processes of the nervous system shall be based on the uniform processes that perceive the incoming sequence or sequences of signal combinations from other processes and generate (while detecting a certain sequence of combinations of interrelated incoming signals) the outcoming sequence of signals through which they influence (interact with) other processes of the nervous system (or the external processes) carrying out the processing of these signals and change of their (uniform processes) properties according to certain rules. The hierarchically higher processes of the nervous system can be implemented through the interaction of the specified uniform processes.

\subsection{Rules and Principles of Functioning of Minimal Uniform Functional Process of Nervous System Based on Regularities in Interaction of Processes of the Universe}

The properties of the minimal uniform functional process of the nervous system described in this section are shown (for notational simplicity) primarily on the basis of the analysis of the interaction and the interdependence of hierarchically self-adjusting nervous system's processes that get influences from other processes of the nervous system and send influences to other processes of the nervous system. However, according to the above $(3.1,4.2,5.4,6.1)$, the described properties of the minimal uniform functional process of the nervous system are also true (taking into account specific differences) for the nervous system's processes that belong to the blocks of preprogrammed reactions (4.3) and their properties and for the nervous system's processes that do not receive signals from other processes of the nervous system (receptors) or do not send the signals to other processes of the nervous system (generators of outcoming signals).

Also, the properties of the minimal uniform functional process of the nervous system described in this section are shown (for notational simplicity) primarily on the basis of the analysis of the interaction and the interdependence of hierarchically processes of the universe. However, according to the above (3.1, $4.2,5.4$ ), the described properties of the minimal uniform functional process of the nervous system are true for the properties of these processes of the universe.

6.2.1. Optimization of Properties (Set of Subprocesses and their Properties as well as Interrelation and Interrelationships) of Processes of Nervous System that Provide Detection of Interrelated Sequences of Signal Combinations by Interrelated Sequences of Signal Combinations Detected by Them

Each process of the nervous system shall detect the interrelated sequences of combinations of influences (signals) from the processes that influence it and (under these influences) generate influences on other processes (6.1.1). This function is implemented through the interaction of the subprocesses that form each process of the nervous system and, therefore, depend on the set of these subprocesses, their 
properties and the interrelation among them, i.e. on the properties of this process (3.1). As each process detects a certain interrelated sequence of signal combinations, its properties (the set of subprocesses, set of properties and interrelationships of these subprocesses) that implement this detection shall be the ones that ensure the detection of this interrelated sequence of signal combinations. Taking into account the optimization of the properties of the processes of the nervous system in order to ensure the minimization of the utilization of the resources (5.3.3), the properties that ensure the detection shall be optimized for detection of a certain interrelated sequence of signal combinations that a certain process of the nervous system detects. The optimization of these properties shall be carried out according to certain rules that are determined by other properties of the processes of the nervous system that, in their turn, are predetermined in the previous generations of the species of the living organism to which the nervous system belongs and transferred to it through the hereditary mechanisms (5.2).

\subsubsection{Variability of those Properties (Set of Subprocesses and Their Properties as well as Interrelation and Interrelationships) of Processes of Nervous System that Provide Detection of Sequences of Signal Combinations}

The detection of the sequences of signal combinations is carried out through certain properties (set of subprocesses and their properties as well as interrelation and interrelationships) of the nervous system's processes, which shall be optimized in order to implement the detection of the sequences of signal combinations that correspond to the process detected (6.2.1). Beforehand, during the initial formation of a certain process of the nervous system, it is not known which sequence of signal combinations will be detected by this process of the nervous system. Thus, the complete set of properties (the set of subprocesses, set of properties as well as interrelation and interrelationships of these subprocesses) required for detection of this sequence of signal combinations is not known. Therefore, these properties shall change during the existence of the nervous system's process to which they belong in order to ensure the detection of a certain sequence of signal combinations. The change of these properties shall be carried out according to certain rules that are determined by other properties of the processes of the nervous system that, in their turn, are predetermined in the previous generations of the species of the living organism to which the nervous system belongs and transferred to it through the hereditary mechanisms (5.2).

For the preprogrammed systems, the detected by them sequence of signal combinations can be known (completely or partially) beforehand.

\subsubsection{Detection of Interrelation in Sequences of Combinations of Signals only from Processes with which there is Interrelation (there is Direct or Indirect Interaction)}

Any process of the universe can influence other process of the universe only through the direct or indirect interaction with it (4.2.1). Thus, to detect the interrelation in the sequences of combinations of influences (signals) from other processes, every process of the nervous system shall directly or indirectly interact with these processes, i.e. be, in a certain way, interrelated with them. Moreover, this interrelation shall have such a character (such properties - the set of subprocesses, set of interrelationships and properties and values of characteristics of properties of these subprocesses - 3.1) to ensure the transfer of the influences (signals whose interrelation of sequences of combinations is being detected) from one process to the other according to certain rules that allow to detect the interrelation of sequences of combinations of such influences.

Hereinafter, when describing the interrelation between the processes of the nervous system that is present during the direct detection of sequences of signal combinations, the described in this subsection type of interrelation will be meant primarily if otherwise is not specified additionally. 
6.2.4. Stability of Interrelation/Interrelationships, through which Detection of Interrelated Sequences of Signal Combinations is Implemented, between Processes of Nervous System and Stability of Their Properties

In order to ensure achievement of the TEFs of the organism in a certain situation, the processes of the nervous system, while detecting this situation in the form of the sequences of combinations of incoming signals, shall provide certain sequences of combinations of outcoming signals to other processes (6.1.1). Moreover, taking into account the definition of the nervous system (4.1.3), every time in case of repetition of one and the same situation a stable correspondence between the incoming and outcoming sequences of signals shall be maintained as long as in this situation ensured the detection of all external processes that influence the nervous system's processes and are critical for achievement of TEFs of the organism and outcoming signals that contribute to the efficient and effective achievement of TEFs of the organism in this current situation are generated. As the generation of the outcoming signals by every process of the nervous system depends on the detected by it incoming signals (6.1.1), and the detected incoming signal depends on the interrelation of this process with other processes (6.2.3), in order to ensure the described stable correspondence between the incoming and outcoming sequences of signal combinations, it is necessary to provide a certain stability of interrelation, i.e. the availability of interrelationships (3.1) between the processes of the nervous system and the stability of those properties of these processes that determine the influence of signals received through these relations on the signal generated by the nervous system's process that receives these signals.

\subsubsection{Variability of Interrelation/Interrelationships, through which Detection of Interrelated Sequences of Signal Combinations is Implemented, between Processes of Nervous System and Variability of Their Properties}

The environment (the universe or its part) in which a living organism exists and the living organism itself are the dynamic (variable) system of interacting hierarchical processes (3.1). Changes can occur in these systems as a result of interactions: new hierarchical processes can form and change, previously existing processes can transfer to other ones or break up (3.1). In order to ensure the efficient and effective achieving TEFs of the organism, taking into account the changes of the processes of the universe, the processes of the nervous system shall provide the detection of the new processes (or the changed, previously detected by them processes) and the new properties of the new or old (previously detected) processes and the generation of outcoming signals that will contribute to the efficient and effective achieving TEFs of the organism. As the generation of outcoming signals by every process of the nervous system depends on the detected by it incoming signals (6.1.1), and the detected in certain repeated situation incoming signal depends on the interrelation of this process with other processes (6.2.3), in order to ensure the described adaptation to the changed situation (detection of completely new or changed sequences of signal combinations and generation of the same as previously or the new sequences of combinations of outcoming signals), it is necessary to provide the variability of interrelationships between the processes of the nervous system, and, thus, to provide the variability of subprocesses and properties of the nervous system's processes that determine the specified interrelationships between these processes of the nervous system.

\subsubsection{Combining of Stability and Variability of Interrelationships, through which Detection of Interrelated Sequences of Signal Combinations is Implemented, and Corresponding Properties of Processes of Nervous System}

As specified above, every process of the nervous system shall ensure the stability (6.2.4) and variability (6.2.5) of its interrelationships with other processes of the nervous system and those their properties that ensure the detection of interrelated sequences of signal combinations. The interrelationships of the nervous system's processes with other processes and the rules of their change are determined by the properties of the processes (3.1). Therefore, all processes of the nervous system shall have such properties that will implement such rules of their change that will ensure both conditions: stability and variability of interrelationships and properties that ensure the detection of the interrelated sequences of signal combinations. Moreover, the properties of the nervous system's processes that 
implement the specified rules shall be set when creating these processes and determined by the natural selection in the previous generations (5.2.3).

\subsubsection{Signal Generated by Processes of Nervous System as Indicator of Probability of Interaction with Certain Process with Certain Properties and Values of Characteristics}

As specified above (4.2.2), all processes of the universe are perceived by the processes of the nervous system in the form of the unique sequences of combinations of interrelated influences from these processes. Moreover, the processes of the nervous system will get influences only from a part of the processes that form a certain process of the universe (some subprocesses will interact with one another or with other processes of the universe not interacting, directly or indirectly, with the processes of the nervous system). Taking into account the above described incompleteness of data about the current state, based on the sequence of combinations of interrelated signals detected by the processes of the nervous system, it is possible to determine only the probability of the fact that the interaction with a certain process, with certain properties and values of characteristics is taking place in the current situation. Thus, the interrelated sequences of signal combinations detected by the processes of the nervous system and, therefore, the influences (signals) generated by them represent only the probability of the fact that the interaction with a certain process is taking place currently. And in every such case, this detected probability can be more, if the more number of the interrelated sequences of signal combinations generated during the interaction with this detected process influences in this case the nervous system's process that detect this unique process, and the more unique (emerge only or most often during the interaction with this process) these sequences of signal combinations are exactly for this detected process. The value of the probability itself can be from 1 (absolute certainty of determination of a certain process with which the interaction is taking place) to 0 (absolute uncertainty of a process with which the interaction is taking place).

\subsubsection{Signal Generated by Processes of Nervous System as Indicator of Similarity of Process (or Certain Property, or Value of Process Characteristic) with which Interaction is Taking Place Currently to Process (or Certain Property, or Value of Process Characteristic) to whose Detection this Process of Nervous System Adjusted}

The hierarchically higher (from the initial level) processes in the universe are mainly similar processes with a different level of similarity (3.1). The processes of a living organism and its nervous system (as the processes of substantially higher than the initial level hierarchy) interact, during their existence, mainly with the similar processes and will perceive these processes in the form of the similar interrelated sequences of signal combinations (4.2.2). Taking into account the similarity of the processes of the universe, in every case, it is possible to determine only the similarity of a certain process with which the interaction is taking place in a certain current situation to another process with which the interaction took place, is taking place or will take place. Therefore, as the processes of the nervous system adjust themselves to the detection of the processes with which they interact (4.1), during every new interaction with the processes of the universe, they can detect only the similarity of the processes with which the interaction is taking place currently to the processes with which the interaction took place in the past cycles of interaction (the similarity to the processes to the detection of which the processes of the nervous system adjusted during the previous interactions with this or other similar to it processes). I.e. every process of the nervous system, when perceiving a certain sequence of signal combinations (4.2.2), determines the level of its similarity to that sequence of signal combinations to the detection of which this process of the nervous system adjusted as a result of the previous interactions with the processes of the universe and generates (at this determination) the influences (signals) that represent the level of their similarity that is a reflection of the level of similarity of the process with which the interaction is taking place currently to a certain process with which the interaction took place in the past. And in every such case, this detected similarity is the more, the more the number of the interrelated sequences of signal combinations is and their properties will coincide to a greater extent with those interrelated sequences of signal combinations and with those their properties that were during the interaction with the similar processes in the past. The value of similarity itself can be from 1 (completely similar) to 0 (completely 
different).

6.2.9. Signal Generated by Each Process of the Nervous System as Combination of Indicators of Probability of Interaction with Certain Process (with Certain Properties or Values of Characteristics) and Value of its Similarity to Process (Certain Properties or Values of Characteristics) to Detection of which this Process of the Nervous System Adjusted

The processes of the nervous system generate the signals that simultaneously reflect the similarity of the detected by them process to the process, to the detection of which they adjusted as a result of the previous interactions with the processes of the universe (6.2.8), and the probability of the fact that the interaction is taking place exactly with this process (6.2.7), thus, the value of the signal generated by each process of the nervous system simultaneously depends on these two indicators. I.e. the more number of the interrelated sequences of signal combinations generated during the interaction with this detected process influence in the current situation the nervous system's process that detects a certain unique process of the universe, and the mote unique (emerge only or most often during the interaction with this process) these sequences of signal combinations are exactly for the specified detected process and their properties coincide to a greater extent with those interrelated sequences of signal combinations and with those properties that were during the interaction with the similar processes in the past, the more value of the signal should be. The signal itself will change the value from the absolute uncertainty of the process of the universe with which the interaction is taking place and its complete difference from the process to whose detection a certain (the one that generates the specified signal) detecting process of the nervous system adjusted (0) to the absolute certainty of detection of a certain process of the universe with which the interaction is taking place and its complete similarity to the process to whose detection a certain (the one that generates the specified signal) detecting process of the nervous system adjusted (1).

Hereinafter, when describing detection of the process of the universe by the process of the nervous system and the corresponding signal generated by this process of the nervous system at this detection (if otherwise is not specified), the term "process determination accuracy" will be used, which will represent the combination of indicators of probability and similarity during the process detection.

\subsubsection{Types of Influences (Signals) of Processes of Nervous System on Other Processes}

The signal generated by the process of the nervous system to influence other processes reflects the determination accuracy of the process of the universe detected by this process of the nervous system (6.2.9). However, the character of the influence of this signal on other process of the nervous system shall be determined by the functions that implement the interacting (the one that generates and the one that perceives the signal) processes of the nervous system. The types of the character of the influence of one process of the nervous system on another one shall contain such interactions that will allow to implement the functions of the organism's nervous system (4.1.3). Taking into account the functions of the processes of the nervous system that they implement during the implementation of the functions of the nervous system (the detection of the interrelated sequences of signal combinations and the generation of the influences, which reflect the determination accuracy of the detected process, on other processes - 6.1.1, 6.2.9), the main possible types of influence of one process of the nervous system on another one are the following:

- Activation of another process of the nervous system (increase of value of the signal generated by it about the determination accuracy of a certain process) during the detection of a certain process. The process that is detected by the activating process of the nervous system is a subprocess of the process detected by the activated process of the nervous system or together with it is a part of a certain hierarchically higher process.

- Deactivation of another process of the nervous system (decrease of value of the signal generated by it about the determination accuracy of a certain process) during the detection of a certain process. The process that is detected by the deactivating process of the nervous system is a process that is a mutually exclusive competitor to the process that is detected by the deactivated process of the nervous system or is a part of a certain hierarchically higher process that does not contain the process detected by the deactivated process of the nervous system. 
- Modulation of interaction of other processes of the nervous system (weakening or strengthening of influence that caused by the signal transmission from one process of the nervous system on another and/or strengthening or weakening influence on the mechanism of strengthening or weakening of the interrelationship between these processes) during the detection of a certain process. The nervous system's process that is exposed to the modulating influence shall adjust itself to the detection of a certain process that is implemented only in case of presence/absence of another process that is detected by the nervous system's process that makes the modulating influence.

The signal generated by the process of the nervous system for the influence on other processes (regardless of the influence type) reflects the determination accuracy of the process of the universe that is detected by this process of the nervous system and change within the range from 0 to 1 (6.2.9). The influence made by one process of the nervous system on other one depends on the value of the signal and on the properties of the interrelationship (the set of subprocesses, set of interrelationships and properties and values of characteristics of these subprocesses) between these processes $(3.1,4.2 .1)$ through which the specified influence is transferred. Thus, the influence made by one process of the nervous system on another process of the nervous system (regardless of the influence type) change within the range from 0 to a certain maximum value (maximally possible influence that can be made through the available interrelationship of the processes in case of the maximal signal about the determination accuracy of the process detected by the influencing process of the nervous system, which equals 1 ).

The functions of the processes of the nervous system are determined by the properties of the processes of the nervous system, which, in their turn, are set while creating the organism's nervous system (5.2), therefore, the properties of the processes of the nervous system that determine the character of their interaction (determine the influence types) are predetermined as well by the natural selection in the previous generations of the species to which this living organism belongs and transferred to the specified organism through the hereditary mechanisms.

\subsubsection{Dependence of Types of Influences (Signals) between Processes of Nervous System on Interrelation of Processes of the Universe Detected by Them}

The processes of the universe, according to the initial assumption (3.1), are the set of the interrelated characteristics, which change according to certain (unchangeable) rules. By interacting with one another, these processes can form the hierarchically higher processes (of various levels of similarity and/or identity) within the limits of which they have a certain interrelation between themselves (3.1). This interrelation of the properties and the values of characteristics of the processes of the universe and the processes themselves (within the limits of the hierarchically higher processes) are detected by the processes of the nervous system as the sequences of combinations of interrelated signals (4.2.2). Taking into account the described interrelation of the available set of characteristics of the process or the set of the processes (within the limits of the hierarchically higher process) and the identity and similarity of the processes of the universe (3.1), the detection of some part of the characteristics from this set or a certain part of the processes (subprocesses) within the limits of a certain hierarchical process increases the probability (6.2.7) of detection of certain other characteristics or processes that are included to this set or, accordingly, decreases the probability of detection of characteristics or processes that are not included to this set.

In case a certain process of the nervous system detects a certain sequence of signal combinations that influences it when interacting with a certain process of the universe during the interaction with which the probability increases that the interaction with other processes of the universe is taking place in a certain current situation, the generated by the nervous system's processes that detect these processes of the universe signals about the accuracy of their determination (6.2.9) shall be increased. The increase of the value of the generated by a certain process of the nervous system signal about the accuracy of determination of a certain process of the universe can be carried out with the activating influence on it from another process of the nervous system (6.2.10). Therefore, when a certain process of the nervous system detects a certain sequence of signal combinations that influences it when interacting with a certain process of the universe during the interaction with which the probability increases that the interaction with other processes of the universe is taking place in a current situation, the nervous system's process that detects this sequence of signal combinations can have the activating influences on other nervous system's 
processes that detect the specified processes of the universe interrelated with the process of the universe detected by this process of the nervous system.

In case a certain process of the nervous system detects a certain sequence of signal combinations that influences it when interacting with a certain process of the universe during the interaction with which the probability decreases that the interaction with other processes of the universe is taking place in a current situation, the generated by the nervous system's processes that detect these processes of the universe signals about the accuracy of their determination (6.2.9) shall be decreased. The decrease of the value of the generated by a certain process of the nervous system signal about the accuracy of determination of a certain process of the universe can be carried out with the deactivating influence on it from another process of the nervous system (6.2.10). Therefore, when a certain process of the nervous system detects a certain sequence of signal combinations that influences it when interacting with a certain process of the universe during the interaction with which the probability decreases that the interaction with other processes of the universe is taking place in a current situation, the nervous system's process that detects this sequence of signal combinations can have the deactivating influences on other nervous system's processes that detect the specified processes of the universe.

The influence of activating and deactivating signals that are transmitted through the interrelationships between the processes of the nervous system, which detect the processes of the universe, increases the number of unique interrelated sequences of signal combinations generated when interacting with these detected processes of the universe in a certain current situation and, thus, increases the accuracy of determination of these processes in this current situation $(6.2 .7,6.2 .8,6.2 .9)$.

The activating and deactivating influences can be implemented thanks to both the properties of the direct interrelationship (6.2.3) between two processes of the nervous system and indirectly through the additional nervous system's processes that, perceiving the activating influences from second process of the nervous system, will implement the deactivating influences on the first process of the nervous system.

\subsubsection{Dependence of Characteristics of Interrelationships of Interacting Processes of Nervous System on Statistical Interrelation of Influences Exerted by Them (Signals Generated by Them) and Values of These Influences (Signals) about Accuracy of Processes Determination}

Every process is detected by the processes of the nervous system as the interrelated sequence of combinations of individual signals (4.2.2) that the processes of the nervous system, excluding receptors, get from other processes of the nervous system when interacting with them (6.2.3). The processes of the universe, except for the processes of the initial level, are composed of the subprocesses that interact with one another (3.1). Every process of the nervous system gets influences from other processes that represent the accuracy of determination of interaction with a certain process of the universe $(6.2 .7,6.2 .8,6.2 .9)$, which can be both the subprocess of the process that is detected by this process of the nervous system and the subprocess of another process that is not detected by this process of the nervous system. The more unique a certain subprocess is for the process detected by a certain process of the nervous system, i.e. the more frequently this subprocess interacts with the processes of the nervous system as a part of this detected process (the influence exerted by this subprocess on the processes of the nervous system is a part of the interrelated sequence of combinations of influences on the processes of the nervous system that emerge during their interaction with the specified detected process — greater statistical interrelation of the process and subprocess), the more the probability that in the presence of the signal about the interaction with this subprocess, the interaction is taking place with a certain detected process of the universe or the process similar to it.

The accuracy of determination of interaction with a certain process of the universe is reflected in the signal (6.2.9) generated by the nervous system's process that detects this process, and the accuracy of determination of interaction with a certain subprocess, therefore, is reflected in the signal that influences the specified process of the nervous system from the nervous system's process that detects this subprocess.

Thus, the more unique a certain subprocess is for a certain detected process (the more they are statistically interrelated), the more frequently, the influence exerted by the nervous system's process that detects the specified subprocess on the nervous system's process that detects the specified process, to which this subprocess is included, will be a part of the sequences of combinations of influences that influence second process of the nervous system (during the interaction of the nervous system's processes 
with it) and result in the generation of a higher signal about the accuracy of determination of the process detected by second process of the nervous system. Besides, the more unique a certain subprocess is for a certain detected process (the more they are statistically interrelated), the bigger the influence shall have the signal from the nervous system's process that detects this subprocess on the signal generated by the nervous system's process that detects the interaction with the mentioned process (to which this subprocess is included) and characteristics of this signal that reflect the accuracy of determination of interaction with the specified detected process. As the specified influence depends on the properties of the interrelationship (on the set of subprocesses, set of interrelationships and properties and values of characteristics of these subprocesses - 3.1) of the interacting processes of the nervous system, these properties shall depend on this statistical interrelation of the influences exerted by them (signals generated by them) and the values of this generated signal (influences) about the accuracy of the process determination. I.e. processes of the nervous system shall change the properties of the interrelationship with other processes of the nervous system to ensure the strengthening of influences from the relations with the processes, the influences (signals) from which form the statistically interrelated sequences of signal combinations, and, accordingly, the weakening influences from relations with those processes, influences from which do not form them.

Note. When the processes of the nervous system detect the processes of the universe with certain values of characteristics, these processes of the nervous system shall adjust themselves to the generation of the maximal signal about the accuracy of determination of the corresponding process of the universe by them not with the maximal value of the signal from the process that detects the value of the corresponding characteristic, but with the value that is statistically most frequently is a part of the interrelated sequence of combinations of influences on the process of the nervous system that emerge during its interaction with the specified process of the universe, detected by it.

\subsubsection{Competitiveness of Interrelationships - Optimization of Relations and Properties of Processes of Nervous System as a Result of Limitation of Their Resources}

The nervous system of the organism has the limitation of its capabilities (5.3). Therefore, every process of the nervous system has the limitation of its capabilities and, thus, has the limitation in the number of possible relations with the processes that influence it and the number of possible relations with the processes that it has the possibility to influence. Besides, every process shall change the properties to ensure the strengthening of influences from the relations with the processes, the influences (signals) from which form the statistically interrelated sequences of signal combinations, and the weakening influences from relations with those processes, influences from which do not form them (6.2.12). Therefore, during the functioning of the nervous system's processes, they shall change by weakening and breaking the relations (by weakening, reducing the number or breaking completely the subprocesses that implement them) with the processes, the sequences of combinations of influences from which are less statistically interrelated with the influences from other processes influencing them, and strengthening the relations (by strengthening and/or increasing the number of the subprocesses that implement them) with those processes, the sequences of combinations of influences (signals) from which are more statistically interrelated with the influences (signals) from other nervous system's processes influencing them.

The properties of the interrelationship between two processes and the value of the signal (influence) that can be transmitted by the influencing process of the nervous system to the process that it influences depend on the properties of both these processes of the nervous system (3.1, 4.2.1). In its turn, the belonging of the signal transmitted through this interrelationship to the statistically interrelated sequence of combinations of signals is determined by the nervous system's process that receiving this signal (6.1). Therefore, in order to provide the required to change properties of the specified interrelationship changes in the nervous system's process that transmitting the signal, it is necessary to transmit the reverse influence from the nervous system's process receiving the signal to the nervous system's process transmitting this signal through the specified interrelationship. 


\subsubsection{Accidental Establishment of New Interrelationships between Processes of Nervous}

System with Further Strengthening or Weakening/Breaking of These Interrelationships

Every process is detected as the interrelated sequence of combinations of individual signals (4.2.2) that the processes of the nervous system, except for the receptors, get from other processes of the nervous system when interacting with them though the available interrelationships (6.2.3). Therefore, the determination of the belonging of the influence from a certain process of the nervous system on another process of the nervous system to the interrelated sequence of signal combinations detected by second process can be made by it only after establishing the interrelationship between these processes of the nervous system $(6.2 .12,6.2 .13)$. Thus, the establishment itself of the interrelationships is of random character (it is not determined in advance whether the influence through a new interrelationship from a certain process of the nervous system will form, together with the influences from other processes of the nervous system, the interrelated sequence of signal combinations to whose detection the nervous system's process that receives these influences can adjust itself) with the further strengthening or weakening and breaking of this interrelationship (6.2.12, 6.2.13).

The detection of the sequences of signal combinations is carried out through certain properties (the set of subprocesses, set of interrelationships and properties of these subprocesses) of the nervous system's processes that change (6.2.2) and optimize (6.2.1) in order to ensure the ability of detection of the sequences of signal combinations that correspond to the process detected. The interaction of the processes of the nervous system is implemented thanks to their certain properties (3.1) that ensure the interrelationships between them and change in order to provide the detection of the sequences of signal combinations (6.2.5). Therefore, taking into account all of the foregoing in this subsection (6.2.14), the process itself of implementation of changes of properties of processes of the nervous system that lead to formation of interrelationships between the nervous system's process that exerts influence and the subprocesses that detect the sequences of signal combinations and belong to the nervous system's process experiencing the influence has a random character with the further strengthening or weakening and breaking of the interrelationships implemented thanks to these properties and the corresponding strengthening or weakening and elimination of the properties thanks to which are implemented these interrelationships and the detection of belonging of the signals from them to a certain sequence of signal combinations to whose detection the nervous system's process that receives these signals adjusts itself (6.2.12, 6.2.13).

\subsubsection{Optimization of Process of Establishment of Interrelationships between Processes of Nervous System Thanks to Their Additional Interrelation that does not Provide Detection of Interrelated Sequences of Signal Combinations}

The nervous system's processes that implement the functions of the nervous system, in addition to the interrelation by means of which the signals (the interrelation of sequences of combinations of which is detected by processes of the nervous system - 6.2.3) transmission is carried out can be interrelated in another way both with one another and with other processes that do not implement the key functions of the nervous system (ensure the functioning of the nervous system's processes and others - 4.1.3). That organism will achieve the target evolution functions in a more efficient way whose nervous system will consume fewer resources during its functioning under otherwise equal conditions (3.2). Thus, the processes of the nervous system shall have the properties that will provide such rules of their interaction (direct or indirect) thanks to the presence of additional interrelation that will lead to the optimization of changes of properties (reduction the use of resources and acceleration of formation of new interrelationships) of the nervous system's processes that lead to the establishment of interrelationships between them (6.2.14) that ensure the detection of the interrelated sequences of signal combinations.

6.2.16. Establishment of Interrelationships through which Deactivating Influence is Transmitted between Processes of Nervous System with Their Subprocesses that are Already Receiving Activating Influences from Other Processes of Nervous System

The detection of the sequences of signal combinations is carried out through certain properties (set of subprocesses and their properties as well as interrelation and interrelationships) of the nervous system's 
processes, which shall be optimized in order to implement the detection of the sequences of signal combinations that correspond to the process detected (6.2.1). In case a certain process of the nervous system detects a certain sequence of signal combinations that influences it when interacting with a certain process of the universe during the interaction with which the probability decreases that the interaction with other processes of the universe is taking place in a current situation, the generated by the nervous system's processes that detect these (other) processes of the universe signals about the accuracy of their determination (6.2.9) shall be decreased (6.2.11). The increase of the generated by a certain process of the nervous system signal about the accuracy of determination of a certain process of the universe is implemented thanks to the activating signals from other processes that influence this process of the nervous system $(6.2 .10,6.2 .9)$ through the existing interrelationships $(6.2 .3)$. In its turn, the deactivating influence of one process of the nervous system on another process of the nervous system (through the existing interrelationships - 6.2.3) results in a decrease of the value of the generated by the second process of the nervous system signal about the accuracy of determination of a certain process of the universe $(6.2 .10,6.2 .9)$. Therefore, in order to reduce the generated by a certain process of the nervous system signal about the accuracy of determination of a certain process of the universe detected by it, the nervous system's processes that exert the deactivating influence on it shall (directly or indirectly - 4.1.1) influence those subprocesses of this process of the nervous system that detect the sequence of signal combinations that also (directly or indirectly) receive the activating influences from other processes of the nervous system.

\subsubsection{Establishment of New Interrelationships between Processes of Nervous System Thanks to Changes in Those Nervous System's Processes that will Exert Influence on Another Process of Nervous System (after Establishment of Interrelationship between Them)}

The properties of the process of the nervous system that provide the detection shall be optimized for detecting a certain interrelated sequence of signal combinations detected by this process of the nervous system (6.2.1). Therefore, the changes of these processes shall be the ones that ensure the detection of the interrelated sequences of signal combinations. Thus, in order to establish the new interrelationships and to transfer the influences to other processes, each process of the nervous system shall have the set of subprocesses that will perform these functions. Hence, when establishing the new interrelationships (by the time the interrelationship is established), the changes shall be made to those properties (the set of subprocesses and their properties) of the processes of the nervous system that will provide the influence on another processes of the nervous system after the establishment of the interrelationships through which the transfer of the influence generated by other subprocesses of these (those that exert the influence) processes of the nervous system will be implement.

Since effectiveness of the influence of one nervous system's process on another one through a certain interrelationship depends on the properties of both processes (3.1) of the nervous system (the one that exerts the influence and the one that receives the influence), after the establishment of the interrelationship between these processes of the nervous system, the changes of the properties that ensure the transfer of the influence from the influencing process to the influenced process shall be carried out in both processes according to the approaches described above (6.2.12, 6.2.13). Moreover, these changes shall not violate the properties that ensure the detection of the sequences of signal combinations in the process that perceives the new influence through the newly established interrelationship.

\subsubsection{Statistical selection of Process that will be Detected by Certain Process of Nervous System}

As specified above (6.2.14), the processes of the nervous system in a random way establish between themselves the interrelationships that ensure the detection of the interrelated sequences of signal combinations. In the future, these interrelationships are strengthened or weakened depending on the statistical interrelation of the signals from these interrelationships with the signals from other interrelationships that also transfer the influences on the same detecting process of the nervous system and that together lead to the generation of an output signal about the accuracy of determination of the detected process by the specified process of the nervous system $(6.2 .12,6.2 .13)$. Therefore, every process of the 
nervous system adjusts itself to the detection of the interrelated sequence of signal combinations that formed in a random way (when establishing the random interrelationships with the nervous system's processes influencing this process) and whose influence statistically most frequently leads to the generation by this nervous system's process of the highest outcoming signal about the accuracy of determination of the process detected. If the signals that come to a certain process of the nervous system from the randomly established interrelationships do not form the statistically stable sequences of signal combinations (i.e. white noise is detected) that lead to generation of the outcoming signal, this process of the nervous system, thus, will not be able to adjust itself to the detection of a certain sequence of signal combinations (to the detection of a certain process of the universe). If, after the adjustment of the nervous system's process to the detection of a certain sequence of signal combinations, these signals change or new signals emerge (from the newly established relations with other processes of the nervous system) and, in this case, the sequence of signal combinations that most frequently leads to the generation of the highest outcoming signal by this process of the nervous system also change, then its interrelationships with the influencing processes will change for more optimal detection of this (new) sequence of signal combinations according to the previously described rules $(6.2 .12,6.2 .13)$.

The stability of the interrelationships of the nervous system's processes and, therefore, the stability of detection (6.2.4) of a certain interrelated signal sequence (a certain process) will be ensured in such a case as long as the detected process manifests itself as the statistically most frequent sequence of signal combinations that can influence the processes of the nervous system and that can lead to the generation of a higher (compared to other sequences that can influence these processes of the nervous system) signal about the accuracy of determination of the process.

The properties of the processes of the nervous system (the set of the group of subprocesses and their properties as well as values of characteristics - 3.1) that provide the detection of interrelated sequences of signal combinations shall be optimized for minimal use of resources when detecting a certain interrelated sequence of signal combinations (6.2.1). Therefore, the optimization of the use of resources by the processes of the nervous system thanks to the interrelation between them, through which the direct detection of the interrelated sequences of signal combinations (6.2.3) is not implemented, but which reflects the rules of interrelation between the processes of the universe (5.4.1) detected by these processes of the nervous system, shall be implemented thanks to the appropriate interrelation of the groups of subprocesses that transmit the signal about the accuracy of process determination to other processes of the nervous system. As the formation of new interrelationships of detectors occurs randomly (with subsequent their changes based on the statistical selection of detected processes, as stated early in this paragraph), in order to optimize the detection of interrelated processes of the universe or their properties, it is necessary that the groups of subprocesses that transmit the signal about the accuracy of determination of interrelated processes of the universe (which interact according to certain rules, which lead to a high probability of their interrelation with one another - 5.4.1) were more interrelated with one another to be much more likely to form relations that would provide the detection of the interrelated sequences of signal combinations with the same processes of the nervous system, which, therefore, can reveal the interrelation of these detected processes.

\subsubsection{Gradual, Multilevel Change of Characteristics of Interrelationships between Processes of Nervous System}

The processes of the nervous system adjust themselves to the detection of the processes of the universe by forming, strengthening, weakening, and breaking the interrelationships between themselves (6.2.12, 6.2.13). The formation of new ones, strengthening, weakening or breaking of the existing interrelationships between the processes of the nervous system within the statistical selection (6.2.18) of the process requires the use of certain resources of the organism (3.2). And the greater the changes in these processes of the nervous system are (the more subprocesses of the corresponding processes of the nervous system are changed and the greater the value of changes in their characteristics is), the more resources the organism will have to consume to implement these changes under otherwise equal conditions. And the nervous system will be more efficient the properties of whose processes will be the ones that will provide fewer resources consumption for the change of the properties of this nervous system's processes (5.3.3). 
As a result of the constant change of external and internal (with respect to a living organism) processes of the universe $(3.1,6.2 .5)$ and the processes of the nervous system and their interrelationships (6.2.5, 6.2.14), the processes of the nervous system are constantly receiving new/changed sequences of signal combinations through the interrelationships with other processes influencing them. Moreover, it is impossible to determine in advance (without a certain statistically significant number of cycles of interaction of changed processes - 6.2.12) how long-lasting or random/temporary the changes of these incoming sequences of signal combinations are, to whose detection a certain process of the nervous system adjusted, for this process of the nervous system. In addition, in these conditions it is necessary to ensure (with the minimal use of resources) both as fast as possible (in order to provide the fastest possible adjustment to achievement of TEFs under the changed conditions - 4.1.2) adjustment of the processes of the nervous system to the detection of the new/changed sequences of signal combinations and the stability (6.2.4) of adjustment to the detection of the sequences of signal combinations during their random/temporary changes. Therefore, the processes of the nervous system shall have the properties that will ensure fewer resource consumption (5.3.3) to change that properties of these processes that provide their adjustment to the detection of the sequences of signal combinations, with the fastest possible readjustment and stability during random/temporary changes. I.e. have different ways to adjust themselves to the detection of the sequences of signal combinations: with minimal changes and corresponding minimal use of resources during the fast adjustment with the subsequent (with increasing in the number of interaction cycles) larger changes needed to ensure the stability and the correspondingly higher resource consumption. The properties of nervous system's processes shall be the ones so that various ways to adjust themselves implemented by the processes of the nervous system provide an overall minimization of resources consumption for the readjustment and detection of sequences of signal combinations while providing the required level of stability and flexibility of detection the processes of the universe.

\subsubsection{Determination of Adjustment of Processes of Nervous System to Detection of Certain Processes by Given Structure of Interrelation of Processes of Nervous System}

The initial structure of interrelation and interrelationships of nervous system's processes and their properties and the rules of change are predetermined and transferred to the living organism to which this nervous system belongs through the hereditary mechanisms (5.2). Each process of the nervous system, thanks to establishing the interrelationships with other processes of the nervous system $(6.2 .3,6.2 .4)$, adjusts itself to the detection of a certain process by statistical selection of the sequence of signal combinations that most frequently leads to its generation of a higher signal about the accuracy of determination of the process (6.2.18). The interrelationships between the interrelated processes of the nervous system are formed in a random way (6.2.14). The more the processes of the nervous system, are interrelated between themselves, the more the probability of their interaction (3.1) and, thus, the more the probability of random establishment of interrelationships between them (6.2.14) are. Thus, the probability of establishment between the processes of the nervous system of such interrelationships that will lead to more frequent and/or greater in value generation of signals about the accuracy of the process determination (by the nervous system's process that perceives the influences from other processes) is determined by the interrelation of these processes that does not lead to the generation of the signals about the accuracy of the process determination $(6.2 .3,6.2 .18)$ and which is determined by the interrelation of the processes of the universe (5.4.1). Therefore, the possibility of adjustment of a certain process of the nervous system to the detection of a certain process (a certain interrelated sequence of signal combinations) depends on the structure of the interrelation of the nervous system's processes and, thus, is predetermined in the previous generations of the species of the living organism and transferred to it through the hereditary mechanisms (5.2).

\subsubsection{Main and Additional Interrelationships that Transmit Influences on Processes of Nervous System}

Adjustment to the detection of a certain sequence of signal combinations by a certain process of the nervous system depends on a predetermined structure of the interrelation of this process with the processes that transmit the influence on it (6.2.20). The structure of the interrelation of the processes of 
the nervous system is determined by their corresponding properties and the values of characteristics (3.1). Therefore, depending on the values of that characteristics of the processes of the nervous system that provide their interrelations ( which have a certain structure), different levels of interrelationships that can form a certain (influencing) process of the nervous system with the nervous system's process that it influences can be distinguished. The interrelationships with the processes that, according to the structural interrelation of the processes of the nervous system, will be able to exert such influences on a certain process of the nervous system that will lead to the greatest in value and statistically most frequent generation (by this process of the nervous system) of the signal about the accuracy of the process determination, will be referred to as the "main", and the interrelationships that will transmit the influences from other processes — "additional".

\subsubsection{Sequential Adjustment of Processes of Nervous System to Detection of Sequences of Signal Combinations}

Each process of the nervous system adjusts itself to the detection of a certain process by statistical selection of the sequence of signal combinations that most frequently leads to its generation of a higher signal about the accuracy of determination of the process (6.2.18). If the signals that come to a certain process of the nervous system from the randomly established interrelationships do not form the statistically stable sequences of signal combinations (white noise is detected) that lead to generation of the outcoming signal, this process of the nervous system, thus, will not be able to adjust itself to the detection of a certain interrelated sequence of signal combinations (6.2.18) and the signals generated by it will not be statistically stable signals about the accuracy of determination of a certain process. Thus, the process of the nervous system will be able to adjust itself to the detection of a certain sequence of signal combinations only after the nervous system's processes that exert the main (6.2.21) influences on this process of the nervous system adjust themselves to the detection of a certain statistically stable sequence of signal combinations.

\subsubsection{Optimization of Properties (Set of Subprocesses and Their Properties as well as Interrelationships) of Processes of Nervous System that Provide Transmission of Signal about Accuracy of Process Determination to Other Processes of Nervous System to Ensure Signal Transmission without Distortions and at Required Speed}

Each process of the nervous system shall have the certain properties - the set of subprocesses, set of interrelationships and properties, and values of characteristics of these subprocesses (3.1) - to transmit the signal (influence) about the accuracy of determination of the process detected by it. These subprocesses interacting with one another and changing, will establish interrelationship (of the process to which these subprocesses belong) with other processes of the nervous system (6.2.17), transmit the signal to them through the established interrelationships and change as these relationships are strengthened or weakened (6.2.12, 6.2.13). During the implementation of these functions, it is also important to provide the signal transmission speed required for the implementation of efficient and effective (which will allow to achieve TEFs of the organism) interaction with the external (with respect to the nervous system and/or the organism) processes. Moreover, this signal shall be transmitted without distortions to all processes that are influenced by the nervous system's process generating it. I.e. each nervous system's process that is influenced by the specified nervous system's process shall receive such a signal that accurately reflects the information about the accuracy of the determination of the process of the universe detected by the nervous system's process from which this signal is received. Therefore, as a result of the limitation of the resources of the nervous system's processes (5.3), the properties of the subprocesses that transmit the signal about the accuracy of determination of the detected process, their number and the character of interaction shall be optimized (5.3.3) not only for the implementation of the processes of establishment and change of interrelationships and influencing (6.2.17) through these interrelationships on other processes but also for such interaction with one another that allows to transmit (to one or more other processes of the nervous system or the organism) the signal (about the accuracy of determination of a certain process of the universe) generated by the nervous system's process to which these subprocesses belong, as efficiently (with fewer resources consumption) as possible, with the speed required to ensure the effective (such 
which will be sufficient to achieve TEFs of the organism) interaction with the external processes, without distortions.

\subsubsection{Similarity and Difference of Rules of Formation, Strengthening, Weakening and Breaking of Interrelationships between Processes Depending on Type of Influence Transmitted through These Interrelationships}

The processes of the nervous system, generating the signals about the accuracy of determination of the processes of the universe (6.2.9), can exert different types of influences on other processes (6.2.10). The formation, strengthening, weakening and breaking of the interrelationships, through which the influences of all the previously described types are transmitted, between the processes of the nervous system are carried out according to the rules described above in this section (6.2). However, there are several clarifications for nervous system's processes that perceive the modulating and deactivating influences:

1) As the modulating influences change, primarily, other interrelationships between the processes of the nervous system, rather than exert the influence necessary for the current determination by the nervous system's process, on which they exert the modulating influence, the accuracy of interaction with a certain process of the universe, they can be transmitted through interrelation (interrelationship) between the processes that does not provide the detection of the interrelated sequences of signal combinations.

2) As the interrelationships, through which the influences on the nervous system's process that also receives the modulating influences, depend on these modulating influences, their stability shall also depend on these modulating influences. I.e. the stability of the interrelationships of the processes of the nervous system established under the action of the modulating influence, and, therefore, the stability of detection (6.2.4) of a certain interrelated sequence of signal combination (a certain process) with involvement of these interrelationships will be ensured as long as the detected process manifests itself as the statistically most frequent sequence of signal combinations (6.2.18) that can influence the processes of the nervous system and which is supported by the modulating influence and can lead to the generation of a higher (compared to other sequences that can influence these processes of the nervous system) signal about the accuracy of determination of the process.

3) The signals that are transmitted through the deactivating interrelationships (as opposed to the activating interrelationships - 6.2.12) reflect not the detection of the process that indicates of an increase in the accuracy (probability and similarity) of detection of a certain process of the universe (6.2.12), but rather the detection of the process of the universe that indicates of the reduction in the accuracy of detection of a certain process, $i$ e. the reduction of the probability (6.2.7) that there is now an interaction with the process of the universe similar (6.2.8) to a certain process of the universe to whose detection the nervous system's process receiving this deactivating influence adjusted. I.e. the signal that causes the deactivating influence on a certain process of the nervous system indicates of a certain inconsistency with the detected process of the universe (or the similarity of these two processes of the universe detected by different, competing processes of the nervous system - 7.2.8) in the sequence of combinations of activating signals that influence the nervous system's process that detects this process of the universe.

\subsubsection{Variety of Types (Specialization) of Processes of Nervous System, Including Minimal Uniform Ones as a Result of Limitation of Nervous System Resources}

All processes of the nervous system, including the basic minimal uniform processes (6.1.2), implement the same functions (6.1.1). However, each process of the nervous system can implement these functions in different conditions (different complexity of the detected processes, different types of received and created influences - 6.2.10, different complexity and resource intensity of the transmission of the generated signal to other processes, etc.). Taking into account the limitation of the resources of nervous system's processes, their properties shall be optimized (5.3.3) for the most efficient (with fewer resources consumption) implementation of their functions taking into account the conditions of their implementation.

The set of processes of the nervous system, their properties and the structure of interrelation and interrelationships, and, thus, the conditions for the implementation of their functions are set while creating the nervous system of the organism (5.2). Moreover, they are optimized according to certain rules 
and regularities present in the processes of the universe (5.4) with which the processes of the nervous system can interact when implementing the strategy of achieving TEFs of the organism to which this nervous system belongs. Taking into account the similarity of the processes of the universe (3.1), the parallelism (5.4.3) and the hierarchy (5.4.2) of the structure of the processes of the nervous system, certain groups of processes of the nervous system (and their subprocesses) can operate in similar conditions. Therefore, the set of the processes of the nervous system shall be divided into the groups (types) of processes that have more similar properties within the group than with the processes from another group. Moreover, these differences in the set of properties shall be the ones that ensure the optimal use of resources by the processes of the nervous system belonging to a certain group (type) in those conditions in which the processes of this group will function.

\subsection{General Structure of Minimal Uniform Process of Nervous System}

\subsubsection{Sequence of Interrelation of those Subprocesses of Nervous System's Processes that Perceive Influences from Other Processes and Detect Sequences of Signal Combinations}

As specified above $(6.1 .1,6.2 .1)$, each process of the nervous system detects a certain interrelated sequence of signal combinations, so its properties (the set of subprocesses and their properties as well as interrelationships) that implement this detection shall be such as to ensure the detection of this interrelated sequence of signal combinations. As the sequence of signal combinations is the set (combination) of signals coming in a certain sequence (for a certain period with certain intervals of time between certain signals), to detect this sequence it is necessary to perceive these signals (which included in this sequence) during a time of duration of this sequence (time from getting the influence from the first signal of the sequence to getting the influence from the last signal of the sequence). Only after receiving the signals for the duration of the sequence, it is possible to determine how the received sequence of signal combinations (the number and the properties of the signals received during this period and the time of their receipt in the sequence relative to the start time of the sequence) coincides with the sequence of signal combinations (the number and the properties of the signals and the time of their receipt in sequence relative to the start time of the sequence) to whose detection adjusted detector that receives this sequence of signal combinations (5.2.2, 5.2.1). Moreover, the group of subprocesses (of the nervous system's process) that detects a certain sequence of signal combinations (6.2.1) shall (3.1, 6.2.9) contain a subgroup of subprocesses on which the resulting signal about the accuracy of the process determination (coincidence of the received and detected sequence of signal combinations) will be formed. This resultant signal (at a certain moment) shall depend on the total value of the influences from the signals (their properties and the time of receipt in the sequence relative to the start time of the sequence) received by the group of detecting subprocesses (which detect the sequence of signal combinations) over the duration of the detected sequence of signal combinations preceding the moment of formation of this resulting signal.

As the processes of the universe constantly (continuously) interact with one another (3.1), the processes of the nervous system constantly perceive the influences from other processes and shall constantly (continuously) determine the presence of those to which detection they adjusted. Therefore, the subprocesses of the processes of the nervous system that are part of the group of subprocesses that detect a certain sequence of signal combinations constantly perceive the influences (signals) from other subprocesses in this group or from other processes of the nervous system and transmit them (directly or indirectly through other subprocesses in this group - 4.2.1) to the subgroup of subprocesses on which the resulting signal about the accuracy of the process determination is formed. Moreover, the transmission of signals shall be carried out in such a way (the time of transmission of each signal in the sequence shall be such) so that to ensure the simultaneous influence (3.1) of all signals of the detected sequence of signal combinations received during the time of duration of this sequence, which preceded the moment of formation of the resulting signal, on the group of subprocesses on which the resulting signal about the accuracy of the process determination is formed. A universal form of the processes interaction that can provide such rules for continuous signal transmission with the maximal unification of the properties of these processes (5.3.2) is the sequential interaction of processes in which the time of the signal passage through the sequentially interacting processes depends on the number of these sequentially interacting 
processes $(3.1,4.2 .1)$. Taking into account the optimization of the use of resources by the processes of the organism (3.2), the system will be more optimal where the transmission of different signals included in the detected sequence of signal combinations will use a single chain of sequentially interrelated subprocesses of the nervous system's process. The length of this chain shall be such as to ensure the maximal time of signal passage equal to the time of duration of the detected sequence. The properties of the subprocesses that form this chain shall provide the summation of the signals transmitted through them according to the same rules as the subgroup of subprocesses on which the resulting signal about the accuracy of the process determination will be formed. The number of subprocesses of the chain that simultaneously transmit the same set of signals shall be such that optimally, with minimal resource consumption, provide the transmission of these signals (6.2.1). The position of the subprocesses that perceive the signals from other processes of the nervous system in the specified chain of subprocesses shall be such that the signal received by them is transmitted by the chain to the group of subprocesses on which the resulting signal about the accuracy of the process determination is formed, for the time equal to the time from receipt of this influencing signal to receipt of the last signal of the sequence (or to a certain value, the same for all signals, greater).

The number of the sequentially interrelated subprocesses (the chain length) forming the chain that detects a certain sequence of signal combinations can be reduced by increasing the complexity of the properties of the subprocesses forming this chain, i.e. in the presence of the dependence of the value of the signal that is transmitted by them on the previous signals that were transmitted by them. In this case, the detected sequence of signal combinations will be detected by the chain of subprocesses as the overlay of several sequential parts of the detected sequence of signal combinations.

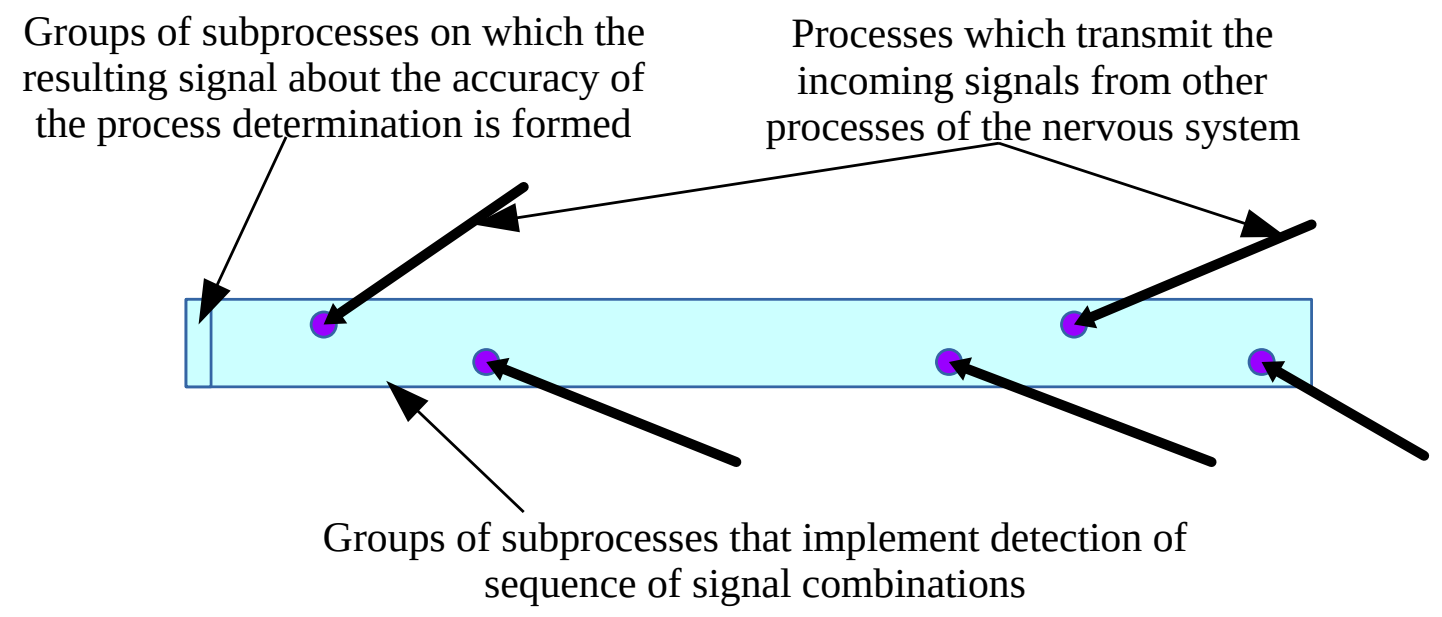

Fig. 3 Schematic Diagram of Construction of Simple Detector of Sequence of Signal Combinations

\subsubsection{Variety of Process Properties and Multivariance of Process Interactions - Parallelism and Branching of Structure of Detector of this Process}

The processes of the universe can interact with one another depending on their properties and form the hierarchically higher processes (3.1), and the bigger the set of these properties in interacting processes, the more interaction options are possible among these processes and the greater the number of different (dissimilar) hierarchically higher processes they can form. Therefore, depending on the variety of the properties, each process of the universe can interact differently with other processes and belong to different hierarchically higher processes. Thus, the nervous system can perceive one and the same process in different interactions with it, in different situations, as the set of different (but those that have a common part that is characteristic of the detected process of the universe in different situation - 6.2.18, 6.2.21) interrelated sequences of signal combinations that can emerge (depending on the set of properties, the current values of its characteristics and the current situation), and, thus, be detected both simultaneously (in parallel) and separately. I.e. in some interactions with this process of the universe, 
several interrelated signal sequences can be detected, in other interactions - only one or a certain part of these sequences can be detected, and in others - the other part of these sequences. Besides, together with each of these sequences of signal combinations, other (additional) sequences of signal combinations can be detected during each of these interactions with the specified process. These (additional) sequences of signal combinations emerge when interacting, in a certain current situation, with the processes of the nervous system of the specified detected process and other processes with which this detected process interacts or to which (as a subprocess) it belongs in this situation. In order to be able to detect the processes of the universe during different interactions with them, the processes of the nervous system shall have such properties that provide the ability of detection of the process (interrelated sequence of signal combinations) as the set of several parallel interrelated sequences of signal combinations and generate the signal about the accuracy of the process determination, with which the interaction occurs, detecting all or a part of these interrelated sequences of signal combinations. Besides, in order to improve the accuracy of the process (6.2.8) determination, the properties of nervous system's processes shall ensure the possibility to detect the additional and, accordingly, additional to additional, etc. sequences of signal combinations that emerge with each of these parallel interrelated sequences of signal combinations as the additional signals (from other processes of the universe related to the specified process of the universe detected in this current situation) that are a part of these sequences.

As each sequence of signal combinations is detected by the set of sequentially interrelated subprocesses of the nervous system's processes (6.3.1), therefore, in order to detect the parallel sequences of signal combinations, the nervous system's processes shall have several parallel sets of sequentially interrelated subprocesses (which shall have a common part), each of which will detect its sequence of signal combinations. And ensuring the detection of additional sequences of signal combinations emerging with a certain of the above specified sequences of signal combinations shall also be carried out by a certain set of sequentially interrelated subprocesses (of the nervous system's process), the outcoming signal from which shall influence the sequence of the interrelated subprocesses that detect the above specified sequence of signal combinations together with which these additional sequences are detected. The determination of the characteristics of the interrelation of these sequences and their influence on the subprocesses (of the nervous system's process) that generate a signal about the accuracy of determination of the detected process is carried out according to the rules described above (6.2.12).

Sequences of group of subprocesses that implement detection of sequence of signal combinations

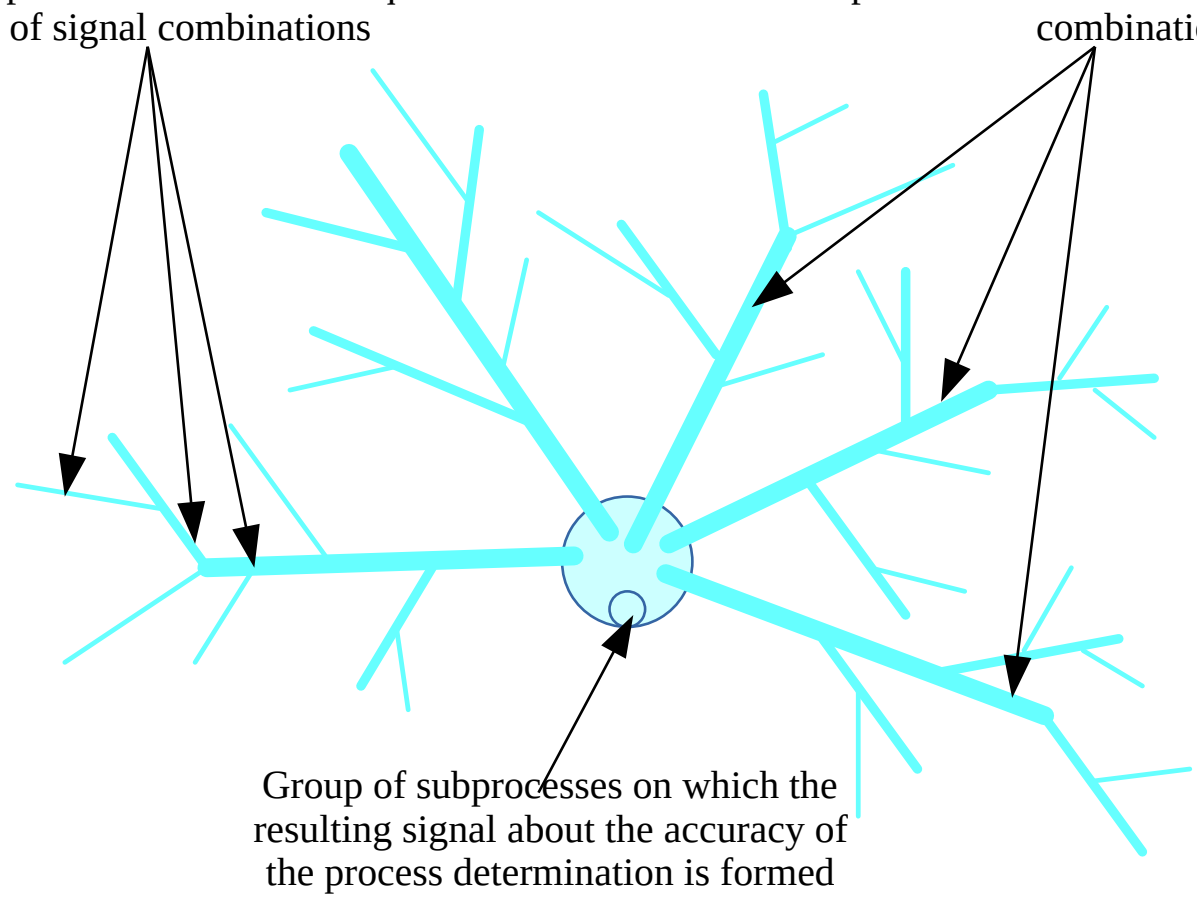

Parallel groups of subprocesses that implement detection of sequence of signal

$$
\text { combinations }
$$

Fig. 4 Parallelism and Sequence of Detectors of Sequences of Signal Combinations of Minimal Universal Process of Nervous System 


\subsubsection{Generated by Process of Nervous System Signal about Accuracy of Determination of Detected Process as Final Sum of All Sequences of Signal Combinations Detected by It}

As specified above $(6.1 .1,6.2 .1)$, each process of the nervous system detects a certain interrelated sequence of signal combinations that represents a certain process of the universe. As a result of this detection, the processes of the nervous system generate the signals (influences on other processes) that reflect the accuracy of determination of the processes of the universe detected by them (6.2.9). The detected process of the universe can influence the processes of the nervous system in the form of a complicated, interrelated sequence of signal combinations, which is detected by a complicated (branched and hierarchical) combination of subprocesses of the process of the nervous system that detect the sequences of signal combinations (6.3.2). Therefore, for the formation of the resulting signal about the accuracy of the process determination, each process of the nervous system shall have the group of subprocesses that will perceive the resulting influences from all the subprocesses of the nervous system's process that detect the sequences of signal combinations. The resulting signal formed on this group of subprocesses shall be transmitted to other processes of the nervous system or the organism.

\subsubsection{Sequence and Branching of Subprocesses that Transmit Signal about Accuracy of Process Determination to Other Processes of Nervous System}

The resulting signal about the accuracy of the process determination is generated by a certain group of subprocesses of the nervous system's process (6.3.3). Therefore, all subprocesses that transmit this resulting signal shall receive influence from the specified group of subprocesses $(3.1,4.2 .1)$ and, thus, interact directly or indirectly with this group of subprocesses to implement the signal transmission process.

As specified above, the processes of the nervous system are constantly determining the presence of the processes to whose detection they adjusted (6.3.1) and, thus, are constantly generating the signal about the accuracy of determination of the process detected by them. Therefore, they shall ensure the continuous transmission of the generated signal to other processes. As the corresponding (6.3.3) group of subprocesses of nervous system's process that generates the signal about the accuracy of the process determination and the nervous system's process that has to perceive this signal can be separated by a certain distance (not to be directly interrelated), to establish the interaction between them through which the signal transmission will be implemented, the necessity can emerge to use the group of the interacting subprocesses to transmit this signal. A universal form of interaction of the processes that can provide such rules for continuous signal transmission with the maximum unification of the properties of these processes (5.3.2) is the sequential interaction of the processes that will form the chain of the required length (3.1, 4.2.1). If the generated signal about the accuracy of the process determination is to be transmitted to several other processes of the nervous system, several chains of subprocesses shall be implemented to transmit this signal.

If the corresponding (6.3.3) group of subprocesses of the nervous system's process that generates the signal about the accuracy of determination of a certain process of the universe, and the group of interrelated nervous system's processes to which it is necessary to transmit this signal, are separated by a considerable distance, in order to optimize the use of the resources when transmitting signal, the parallel chains shall be combined into a single chain that will branch directly next to the group of the processes to which the signal will be transmitted. Taking into account the formation of the structure of the nervous system thanks to simple uniform predetermined (determined by evolution in the previous generations and transferred by the hereditary mechanisms) rules implemented by certain properties of the uniform processes of the nervous system (5.3.2), this chain in each process of the nervous system can be a single chain as the bigger number of the chains will require more complicated rules for the formation of the structure of the nervous system. 
Group of subprocesses that

transmit signal about accuracy of

process determination to a

substantial distance

Groups of subprocesses that transmit signal about accuracy of process determination directly to other processes of nervous system

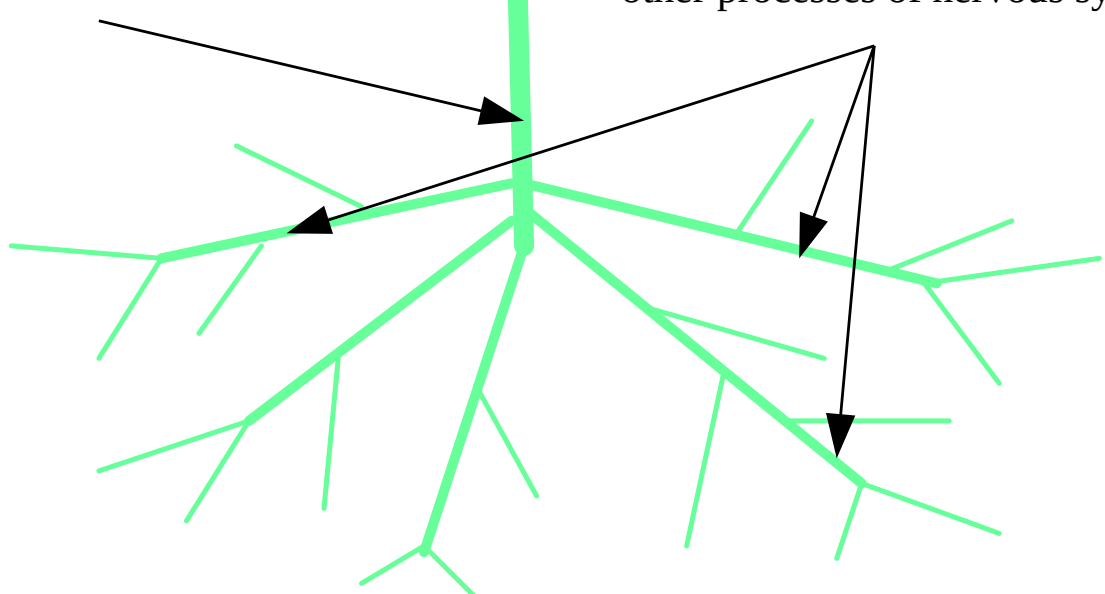

Fig. 5 Structure of Interrelation of Group of Subprocesses which Transmit Signal about Accuracy of Process Determination

\subsubsection{Signal Delay in Interaction of Processes of Nervous System (Process Detection as Long-lasting Process)}

As each process of the nervous system detects and transmits the influences to other processes by means of interaction of sequentially interrelated subprocesses (6.3.1, 6.3.4), the implementation of this process of interaction will take some time that depends on the properties of the subprocesses that form this nervous system's process and the properties (6.3.1) of the sequence of signal combinations detected by this nervous system's process. Therefore, each subsequent nervous system's process will receive the signals from other nervous system's processes, which detect certain processes, after a certain time after the direct influence of the signals coming to these other nervous system's processes.

As specified above, the nervous system is a hierarchical process (4.1.3) that consists of the sets of interrelated hierarchical groups of nervous system's processes, each of which detects certain processes of the universe at a certain level of the hierarchy or certain properties of these processes (5.4). Thus, the described above delay in detecting the processes of the universe by each process of the nervous system will lead to a delay in detecting the processes of the universe of different levels of the hierarchy or their corresponding properties. Moreover, the higher the level of the hierarchy of the detected process or its properties, the more groups of processes of the nervous system will sequentially detect the subprocesses of this process of the universe and/or their properties (5.4.2). Therefore, more cycles of the interaction of the processes of the nervous system belonging to these groups (and their subprocesses) shall be implemented from the process of a direct or indirect (4.2.1) influence of a certain process of the universe (a certain level of the hierarchy) on nervous system's receptors (4.2.2) to detection of this process (or its certain properties) by the corresponding nervous system's processes that detect the corresponding groups of the processes of the universe of the appropriate level of the hierarchy.

\subsubsection{Full Structure of Minimal Uniform Process of Nervous System}

According to the previously described properties (6.2, 6.3.1 - 6.3.4), each minimal uniform process of the nervous system consists of three main groups of subprocesses: a) the group of subprocesses that detects the sequences of signal combinations that occur when interacting with a certain process (6.3.1, 6.3.2); b) the group of subprocesses on which the resulting signal about the accuracy of determination of the detected process is formed $(6.3 .3)$; c) the group of subprocesses that transmits this signal to other 
processes of the nervous system (6.2.17).

The properties of the groups of subprocesses that are included in the minimal universal process and their subgroups can differ depending on the conditions (6.2.25) in which this process of the nervous system functions and the influences it receives from other processes or exerts on other processes (6.2.10). Besides, the set of the properties of the specified groups of subprocesses can be optimized in order to reduce the total consumption of resources when detecting and generating the sequences of signal combinations by the processes of the nervous system as a whole. Such optimization will mean the increase on the complexity (the sets of subprocesses and the variety of their properties) of the subprocesses of the minimal universal process of the nervous system to provide the ability of the detection of more complicated sequences of signal combinations by single minimal universal process of the nervous system. This optimization will reduce the total number of the minimal universal processes required to build the nervous system that can interact (detect or influence) with a certain number of the processes of the universe or increase the number of the processes of the universe with which the nervous system will be able to interact at an unchangeable number of minimal universal processes of the nervous system (5.3).

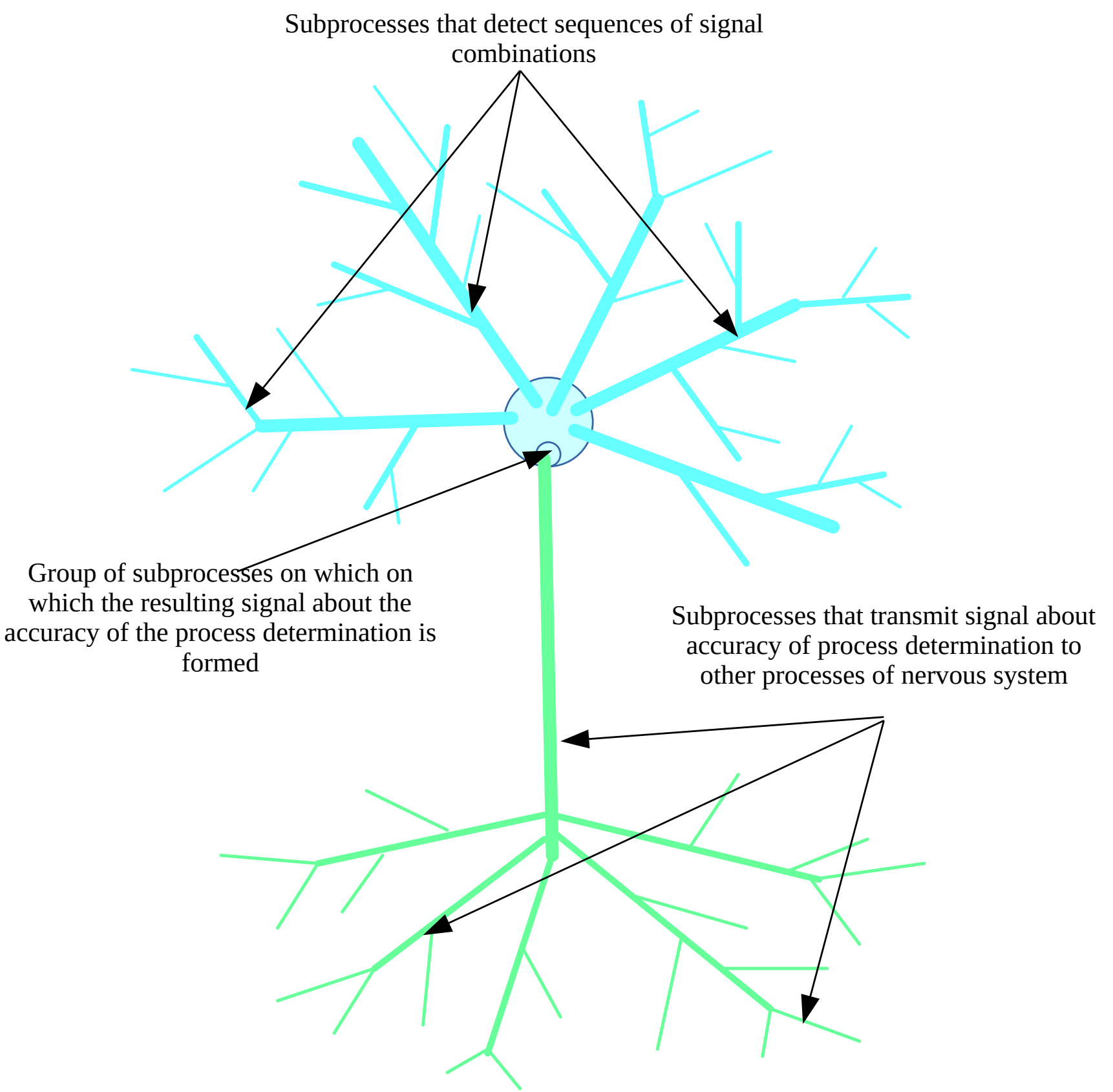

Fig. 6 General Structure of Minimal Universal Process of Nervous System 


\section{Structure and Principles of Functioning and Interaction of Main Functional Blocks of Nervous System}

\subsection{General}

All processes of the nervous system implement their functions perceiving the sequences of signal combinations from other processes and generating the influences on other processes (6.1.1). The minimal nervous system's process that can fully implement the specified functions is the minimal uniform process of the nervous system (6.1.2). Therefore (3.1, 5.3.2), all hierarchically higher processes of the nervous system consist of the set of minimal uniform processes of the nervous system. The properties of the hierarchically higher processes depend on the set and the properties of their subprocesses (3.1). Thus, the set of the minimal uniform processes of the nervous system, their types (6.2.25) and the structure of interrelation and interrelationships within each hierarchically higher process of the nervous system are determined by the functions that are implemented by this process and are predetermined in the previous generations of the species of the living organism, to which this nervous system belongs, and transferred to it through the hereditary mechanisms (5.2).

The self-adjusting (4.3) processes of the nervous system adjust themselves (6.2.18) to detect interrelated sequences of signal combinations that correspond to certain processes of the universe (4.2). The set of the interrelated processes and/or their properties as well as the structure of interrelation and interrelationships, to whose detection the self-adjusting processes of the nervous system can adjust themselves, depends on the set of the processes of the nervous system, sets of their properties, the values of characteristics and the structure of interrelation and interrelationships (5.3.4), which, in their turn, are determined by the strategy of achieving TEFs of the organism (5.2.2) and are predetermined and transferred through the hereditary mechanisms (5.2.1). Therefore, the character of the interrelation among certain processes or their properties, to whose detection certain self-adjusting processes of the nervous system can adjust themselves, also depends on the strategy of achieving TEFs of the organism and is predetermined and transferred through the hereditary mechanisms and can be both more limited (by certain additional types of interrelations) and have no additional limitations. I.e., in addition to selfadjusting nervous system's processes that have the structure of interrelationships that does not impose additional limitations on the detection of different (possible for these nervous system's processes to detect) types of interrelation between the processes of the universe (the sequences of signal combinations - 4.2.2, 4.2.3) to whose detection these processes of the nervous system can adjust themselves, there can be the processes in the nervous system whose structure of interrelation leads to the possibility of their adjusting to detection of only certain (more limited than usual) types of interrelation between the processes of the universe.

\subsection{General Properties, Principles of Construction and Functioning of Generators and Detectors of Nervous System}

Hereinafter, when describing the nervous system's processes that are included to the blocks of analysis/ synthesis of sequences of combinations of signals (detectors and generators respectively), in the first place (unless otherwise stated), the self-adjusting processes of the nervous system shall be meant (4.3).

\subsubsection{Functions of Nervous System's Processes (Detectors/Generators) Forming Blocks of Analysis and Synthesis of Sequences of Combinations of Signals}

As specified above (4.3), the nervous system of the combined type includes the blocks of analysis/synthesis of sequences of combinations of signals consisting of the sets of detectors/generators of sequences of signal combinations. Moreover, the detectors/generators of the sequences of signal combinations are the nervous system's processes that directly implement the detection of the hierarchical processes of the universe (3.1) and generation of the hierarchical sequences of combinations of influences, corresponding to the current-foreseeable situation, on these processes and function according to the rules 
described above $(7.1,6.2)$. These processes form the working in parallel interrelated hierarchical structures of sets of detectors/generators (5.4), which adjust themselves $(4.3,4.1 .3,6.2 .18)$ to the detection of the processes of the universe (with which the nervous system interacts during the existence of the living organism whose part this nervous system is) and adjust themselves $(4.3,4.1 .3,6.1 .1,6.2 .18)$ to the corresponding generation of influences on these processes of the universe. Moreover, these influences shall be such that they are very likely (from the point of view of the previous interactions) to lead to the efficient and effective satisfaction of dissatisfied TEFs of the organism (4.1.3, 5.3.6).

According to the above, the definition of the blocks of analysis (detectors) of sequences of combinations of signals and the blocks of synthesis (generators) of sequences of combinations of signals (4.3) can be formulated as follows:

- The blocks of analysis of sequences of combinations of signals are the working in parallel interrelated hierarchical sets of processes of the nervous system (hereinafter referred to as the detectors) each of which, interacting with other processes (perceiving the influences from other processes), adjusts itself to the detection of a certain sequence of signal combinations, which reflect a certain hierarchical process of the universe or its property, and generates the sequence or sequences of signal combinations, which reflect the accuracy of determination the presence of interaction with a certain process.

- The blocks of synthesis of sequences of combinations of signals are the working in parallel interrelated hierarchical sets of processes of the nervous system (hereinafter referred to as the generators) each of which, interacting with other processes (perceiving the influences from other processes), adjusts itself to the detection of a certain sequence of signal combinations, which reflect a certain hierarchical process of the universe or its property, and generates the sequence or sequences of signal combinations which reflect the accuracy of determination the presence of a certain process. Moreover, each such process of the nervous system (generator) shall detect such process (with such a set of properties and the values of characteristics) that the influence exerted by the generated by the generator signal about the accuracy of determination of this process would lead in the current situation to such interaction of the processes of the universe that will be very likely to lead to the efficient and effective satisfaction of the TEFs of the organism (4.1.3).

\subsubsection{Processes Detected by Nervous System's Processes that are Included to Blocks of Synthesis of Sequences of Combinations of Signals (Generators) as Processes of Influence of Nervous System's Processes on Other Processes of the Universe}

The processes of the nervous system contribute to increase in the probability of achievement of TEFs of the organism by exerting the influences on other processes of the universe, including other processes of the organism, through a direct or indirect interaction with these processes $(4.1,4.2)$. During this interaction, the processes that receive the influence from the processes of the nervous system change the values of their certain characteristics $(3.1,4.2 .1)$ depending on the values of the characteristics of the nervous system's processes influencing them - the generated by these processes of the nervous system signals (4.2) that, in their turn, reflect the accuracy of determination of processes detected by them (6.2.9). This very interaction of the processes, as a result of which this influence is exerted, is also a certain process (3.1) — the process of influence (interaction) of the nervous system's processes on other processes of the universe. I.e. the processes of influence are the processes of interaction of nervous system's processes with other processes of the universe, including other processes of the nervous system, caused and determined by the signals generated by the influencing processes of the nervous system, and lead to such changes in the characteristics of the processes of the universe that, in the current situation, will lead to such change or interaction of the specified processes of the universe (with one another and with other processes of the universe) that is more likely to lead to the efficient and effective satisfaction of the organism's TEFs (4.1.3).

In a certain current-foreseeable situation, each nervous system's process that is the generator detects such a process that the generated by this generator signal about the accuracy of determination of this process exerts such an influence on other processes that, in this situation, will be more likely to lead to satisfaction of the dissatisfied TEFs of the organism (7.2.1). I.e. every nervous system's process that is the generator shall detect such a process during the implementation of which, in the current-foreseeable situation, increases the probability of achievement of TEFs of the organism, moreover, the probability of 
achievement of TEFs in this situation increases as a result of the influences on the processes of the universe caused by the signals generated by the generators.

Thus, the processes of the nervous system contribute to the increase of achievement of TEFs of the organism in a certain current-foreseeable situation exerting the influences on other processes of the universe $(4.1,4.2)$ thanks to the implementation of processes of influence (as noted above), and these processes of influence are the processes during the implementation of which in this situation the probability of achievement of TEFs of the organism increases, and these processes themselves (and, thus, the probability of achievement of TEFs) are caused and determined by the generated by the generators signals about the accuracy of their determination.

Eventually, the generators detect the processes of influence (which are implemented to increase the probability of achievement of TEFs of the organism) of the processes of the nervous system on other processes of the universe, and the signals generated by them are the signals about the accuracy of determination of these processes of influence. Moreover, these generated signals cause and determine these processes of influence, i.e. the course and the result of the interaction of the processes within these processes of influence (required to achieve TEFs changes of the characteristics of the processes of the universe on which the influence is exerted).

The sequences of signal combinations generated by the generators (4.2.3) cause and determine the corresponding sequences of combinations of the processes of influence.

\subsubsection{Difference between Processes Detected by Detectors and Generators of Signals}

The processes of the nervous system (detectors/ generators) that are a part of the blocks of analysis/synthesis of sequences of combinations of signals detect the interactions with a certain process (7.2.1). However, the generators, unlike the detectors, detect the processes that are caused and determined by the signals generated by these (detecting these processes) generators (7.2.2), and the processes that are detected by the detectors do not have such a direct dependence.

\subsubsection{Advance Detection of Processes by Generators of Sequences of Signal Combinations}

The generators detect the processes of influence that are caused and determined by the signals generated by these nervous system's processes (7.2.2). I.e. the processes of influence, at which the probability of achievement of TEFs of the organism is increased, are implemented under the influence of the signals about the accuracy of their determination that are generated by the corresponding generators. When detecting, generating and transmitting to other processes the signals about the accuracy of the determination of processes, a sequential interaction of many subprocesses of the corresponding processes of the nervous system $(6.3 .1,6.3 .4)$ takes place, which is implemented over a certain period of time (a certain number of sequential interactions - 6.3.5). The end result of this sequence of interactions is the process of influence as a result of which the characteristics of other processes of the universe change (depending on the signal obtained as a result of this sequence of interactions - 7.2.2). Therefore, the detection of the processes of influence and the generation of the corresponding signal about the accuracy of their determination shall precede the implementation of these processes themselves and, thus, the detection of the processes of influence on the basis of receiving the influence from them by the nervous system's processes detecting them is impossible.

\subsubsection{Indirect Detection of Processes by Generators of Sequences of Signal Combinations}

The processes of influence that are implemented to achieve TEFs of the organism are the subprocesses of the process of interaction of the processes of the universe whose part the organism is in which these processes of influence are implemented. The increase in the probability of achievement of TEFs of the organism within the specified process (a certain current-foreseeable situation - 4.1.2) will depend on the extent to which the processes of influence implemented by the nervous system in this current-foreseeable situation will contribute to increase of this probability of achievement of TEFs of the organism (4.1). The processes of the universe interact according to the constant rules determined by the sets of their properties and the values of characteristics (3.1), and in one and the same current-foreseeable situation (4.1.2) in order to increase the probability of achievement of TEFs of the organism, it will be necessary to carry out 
the same processes of influence with the same characteristics (4.1.1, 4.2.3, 7.2.2). Therefore, the processes of influence that will lead to the increase in the probability of achievement of TEFs of the organism can be indirectly detected (as a subprocess of process that is the current-foreseeable situation) by detecting the situation in which the detected process of influence, which will be caused and determined by the signal (with certain characteristics) about the accuracy of determination of this process of influence (7.2.2) generated during such detection, will lead to the increase in the probability of achievement of TEFs of the organism.

\subsubsection{Controlled Formation of Sequences of Signal Combinations Detected by Generators}

Each process of the nervous system adjusts itself to detect the sequence of signal combinations (process - 4.2) whose influence statistically most frequently leads to the generation by this nervous system's process of the outcoming signal about the accuracy of determination of the detected process (6.2.18). The process to whose detection every generator adjusts itself depends on the probability of achievement of TEFs of the organism when generating by this nervous system's process (generator) the signal about the accuracy of determination of the specified process (7.2.1). Therefore, at least a part of the signals (the signals through the main interrelationships - 6.2.21) of those influencing every nervous system's process that is the generator shall change so that their influence creates the statistically most frequent sequence of signal combinations during the detection of which this generator will generate such a signal about the accuracy of the process determination that will lead, in the current situation, to such interaction of the processes of the universe that will most likely lead to the efficient and effective satisfaction of the organism's TEFs in this current-foreseeable situation. The other part of the signals (the signals through additional interrelationships - 6.2.21) coming from other processes of the nervous system will strengthen or weaken their relations with this process of the nervous system (generator) according to the rules described above (6.2.12). The relations themselves will be formed and function according to the general rules for the processes of the nervous system (6.2).

\subsubsection{Combination of Nervous System's Processes Exerting Activating and Deactivating Influences, in Detectors and Generators}

As specified above (6.2.11), both the activating and deactivating signals from other processes of the nervous system lead to an increase in the accuracy of determination of the processes of the universe by the processes of the nervous system. Therefore, the detectors and generators shall include the processes that, by perceiving the influence from the nervous system's processes that are a part of other and/or the same as they detectors or generators, will exert both activating and deactivating influences on the nervous system's processes that are a part of other detectors or generators, or on other nervous system's processes that are a part of the same detectors or generators as the specified nervous system's processes. Taking into account the structure of the minimal uniform process of the nervous system (6.3), which underlies the hierarchically higher processes of the nervous system (6.1.2), the specialization of the uniform processes of the nervous system (6.2.25) and the difference in their influences, the activating and deactivating influences on other processes can be exerted by different types of the nervous system's processes that will be a part of detectors and generators.

The list of the processes that are a part of detectors and generators, their properties and the structure of interrelation are predetermined in the previous generations of the species of the living organism to whose nervous system these processes belong and transferred to it through the hereditary mechanisms (5.2, 5.2.2). The relations among these processes of the nervous system will be formed and function according to the general rules for the nervous system's processes (6.2).

\subsubsection{Competitiveness of Detectors at Each Level of Hierarchy}

The blocks of analysis/synthesis of sequences of combinations of signals in the nervous system have a hierarchical structure (5.4.2, 5.4.7). Moreover, each block at each level of the hierarchy consists of a certain number (5.4.8) of detectors/generators working in parallel (5.4.4). Taking into account the similarity of the processes of the universe (3.1), the interrelation in the structure of the processes of the nervous system (5.4.1) and the self-adjustment (self-programming) of the processes of the nervous system 
to the detection of a certain process (6.2.18), in mentioned structure simultaneous adjustment of several parallel interrelated (those at the same level of the hierarchy) detectors (processes of the nervous system) to the detection of one and the same sequence of signal combinations coming to them from the same processes of the nervous system is possible. As a consequence, such duplication will lead to an excessive consumption of resources when detecting the necessary $(5.2 .2,5.3 .4)$ processes of the universe. In order to avoid such duplication and, therefore, to reduce the consumption of resources, these processes of the nervous system working in parallel at a certain hierarchical level shall interact with one another in such a way that the adjustment of each process to detection of a certain sequence of signal combinations would reduce the probability of adjustment to detection of this sequence of signal combinations by other interrelated (adjacent) processes of the nervous system — the competitive adjustment. As such competitive interaction of these processes of the nervous system leads to the change of the adjustment to detection of a certain sequence of signal combinations by a certain process of the nervous system, it shall be carried out through the established interrelationship (6.2.3) where the outcoming (generated) signal about the accuracy of the process determination from one process of the nervous system will influence the detector of sequences of signal combinations of an interrelated (adjacent) process of the nervous system. Moreover, this influence shall have a deactivating character that will reduce the generated by the nervous system's process that receives this influence signal about the accuracy of determination of a certain process of the universe, and/or a modulating (6.2.10) character that will reduce the possibility of adjustment of the process of the nervous system receiving this influence to the detection of a certain sequence of signal combinations in the presence of the specified influence.

The adjustment of generators to detection of certain processes (sequences of signal combinations) is provided by the mechanisms described above (7.2.6).

\subsubsection{Sequential Adjustment of Detectors/Generators to Detection of Sequences of Signal Combinations}

The blocks of analysis/synthesis of sequences of combinations of signals in the nervous system have a complicated branched hierarchical structure, at each level of which the processes of the universe of a certain level of the hierarchy or their properties are determined (5.4.2, 5.4.7). The processes of the nervous system are sequentially adjust themselves to detection of the interrelated sequences of signal combinations (6.2.22). Thus, the detectors/generators of every structural block will be able to adjust themselves to the detection of a certain sequences of signal combinations only after the structural blocks of detectors/generators that exert the main (6.2.21) influences on these detectors/generators adjust themselves to the detection of a certain statistically stable sequences of signal combinations.

The processes of a certain (except for initial) level of the hierarchy are the set of the properties with certain values of characteristics and consist of the processes of the lower level of the hierarchy with certain properties and values of characteristics (3.1). The detection of these processes of a certain (except for initial) level of the hierarchy (the set of their properties and values of characteristics) is carried out as the detection of sequences of combinations of processes of the lower level of the hierarchy, their properties and values of characteristics $(4.2,5.4 .2,5.4 .7)$. Therefore, the adjustment of the blocks of detectors/generators to the detection of the processes of a certain level of the hierarchy, their properties and values of characteristics is possible after adjustment of the corresponding hierarchical blocks of detectors/generators to the detection of the corresponding processes of the lower level of the hierarchy and their properties and values of characteristics.

\subsubsection{Combined Detection of Subprocesses of Detected Process and Transit Signal Transmission}

Detection of a certain process (or a certain process property) requires a certain set of incoming signals from the nervous system's processes that detect the hierarchical processes that are the subprocesses of this process $(5.4 .2,5.4 .7)$. Some of these signals can form the interrelated sequences of signal combinations that can also be used to detect other hierarchical processes or their properties. To optimize the number of the levels of the hierarchy of the nervous system structure in order to reduce the use of resources, the combined intermediate detection of the subprocesses of the detected process can be used. Such detection 
will be implemented by a part of the subprocesses of the nervous system's process that detects a certain process. The received during such detection signal about the accuracy of the subprocess determination is transmitted both to other parts (subprocesses) of the detecting process of the nervous system (detector/generator) and to other processes of the nervous system (detectors/generators) that are parts of other structural blocks of the nervous system.

The signals that come to a certain process of the nervous system and ensure detection by it of a certain process of the universe or its certain property or value of characteristic can also be used to detect other processes of the universe or the properties or the values of characteristics. In this case, their passage to other processes of the nervous system (detectors/generators) can be carried out both directly from the processes of the nervous system (detectors/generators) that generated them, and in transit through the specified process of the nervous system (detector/generator). The choice of transmission method shall be optimal in terms of the use of resources and capabilities and limitations of the available types of minimal universal processes of the nervous system.

The structure of the nervous system of the living organism and detectors/generators that implements the method of signal transmission and combining during the processing of the sequences of signal combinations is predetermined and transferred to the living organism to which this nervous system belongs through the hereditary mechanisms (5.2).

\subsubsection{Complexity of Structure of Individual Detectors/Generators of Sequences of Signal Combinations}

The blocks of analysis/synthesis of sequences of combinations of signals in the nervous system have a complicated branched hierarchical structure (5.4.2, 5.4.7). In this structure, each process of the nervous system (detector/generator), which detects a certain process of the universe, receives and sends the influences from/on many other processes of the nervous system at different levels of the hierarchy. Moreover, these influences can have different character $(7.2 .8,7.2 .10)$ when detecting one and the same process.

All processes of the nervous system are hierarchical processes (4.1.3) that are based on the minimal universal processes of the nervous system $(6.1 .2,7.1)$, which have a certain variety of types and their unification (general and within the types - 6.2.25, 5.3.2) and function according to certain rules and have a certain structure with certain limitations $(6.2,6.3)$. Taking into account the above described branching of interrelationships and the different character of the influences transmitted through these interrelationships within each detector/generator and the limited capabilities and unification of different types of minimal universal processes of the nervous system, each detector/generator shall consist of several minimal universal processes of the nervous system. The number of different types of these processes in each detector/generator and the structure of their interrelationships (both within one detector/generator and within the overall structure of the nervous system) shall be such as to ensure the implementation of the functions of that detector/generator and shall be predetermined and transferred to it through the hereditary mechanisms (5.2).

\subsubsection{Inverse Interrelationships of Detectors/Generators in Blocks of Analysis/Synthesis}

The universe, according to the initial assumption (3.1), is the set of hierarchical processes that have certain properties, which determine the interaction of these processes. These hierarchical processes are detected by the processes of the nervous system (detectors/generators - 7.2.1), which have an interrelated hierarchical structure. Each block of detectors/generators in this structure detects the processes of the universe of a certain level of the hierarchy (5.4.2) as the set of their subprocesses, which influence the nervous system due to their certain properties that can be detected by the receptors of the nervous system (5.4.5, 5.4.6, 5.4.7). Moreover, during the detection of the hierarchical processes, there is both a direct and inverse interdependence. I.e., when detecting a certain combination of the interrelated processes with certain properties and values of characteristics, a certain hierarchically higher process is, therefore, detected and vice versa, when detecting a certain hierarchically higher process, a certain combination of interrelated hierarchically lower processes with certain properties and values of characteristics shall be (with great probability, taking into account the similarity of the processes of the 
universe) detected. I.e. there is both direct and inverse interrelation between the signals generated by the processes of the nervous system during the detection of these processes of the universe (4.2). Thus, the accuracy of determination of a certain process (6.2.9) by a certain detector/generator at a certain level of the structure of detectors/generators can be enhanced by the additional (6.3.2) inverse influences (interrelationships) from detectors/generators that perceive the direct influences from that detector/generator. These (additional) influences make it possible to clarify, on the basis of the previous statistical data, which process is to be detected at this "lower" level detector.

\subsubsection{Activation of Several Detectors/Generators when Interacting with Certain Process}

The universe, according to the initial assumption (3.1), is the set of hierarchical processes that have certain properties, which determine the interaction of these processes. These hierarchical processes are detected by the processes of the nervous system (detectors/generators - 7.2.1), which have an interrelated hierarchical structure. Each block of detectors/generators in this structure detects the processes of the universe of a certain level of the hierarchy (5.4.2) as the set of their subprocesses, which influence the nervous system due to their certain properties that can be detected by the receptors of the nervous system $(5.4 .5,5.4 .6,5.4 .7)$. Therefore, during the interaction of the processes of the nervous system with a certain process of the universe, they will generate the signals about the accuracy of determination of this process (or its subprocesses) at different levels of the structure of the nervous system. The number of detectors/generators that will generate the signals about the accuracy of determination of the specified process of the universe (or its subprocesses) will depend on the number of detected properties thanks to which the interaction takes place and the number of detected subprocesses of that detected process. In addition, at each level of the structure of the nervous system, where the specified processes of the nervous system will generate the signals, the nervous system's processes that detect the processes of the universe similar to the one with which the interaction takes place will also generate the signals (with a smaller value of the signal about the accuracy of the process determination).

\subsection{System of Formation of Main Signals that Influence Formation of Signal Detected by Generators}

\subsubsection{Activation of Nervous System's Processes that Transmit Main Signals to Generators by Signals from Detectors of Processes of Interaction of Organism's Processes with One Another or with Other Processes of the Universe}

Each process of influence (7.2.2) influences the processes of interaction of the processes of the universe (the interaction of the organism's processes with one another or with other processes of the universe). Each sequence of combinations of outcoming signals generated by the generators leading to the implementation of the sequences of combinations of processes of influence shall lead to the increase of the probability of achievement of TEFs of the organism (7.2.1, 7.2.2). At any time, only those nervous system's processes that are necessary to control the degree of the TEF achievement and the processes that are necessary to provide the sequences of combinations of interactions that will allow to achieve the required degree of achievement of TEFs of the organism shall function (5.3.6). Therefore, each process of influence or sequence of combinations of processes of influence shall be implemented only if a certain (the one that can be influenced by these processes of influence) process of interaction of organism's processes with one another or with other processes of the universe is carried out. As the generation of the signals about the accuracy of the determination of processes is carried out by the processes of the nervous system under the influence of the signals coming to them from other processes $(4.2,6.2 .3)$, the main signals coming to the generators (7.2.6) shall come (directly or indirectly) through the interrelationships with the detectors that detect certain processes of interaction (including the processes of interaction during the implementation of which the detectors of dissatisfaction of the organism's TEFs generate the signals - 5.2.5) of the organism's processes with one another or with other processes of the universe the course of which the processes of influence that caused by the signals generated by these generators can influence (hereinafter to define such processes of interaction of the processes of the organism with one another or 
with other processes of the universe, the term "controlled processes of interaction" shall be used). These main signals shall change $(6.2,6.3)$ so that their influence on the generator produces the statistically most frequent sequence of signal combinations during the detection of which this generator will generate such a signal that will lead to the process of influence that will increase the probability of achievement of TEFs of the organism in a certain current situation (7.2.2, 7.2.6). Therefore, the processes that generate these main signals shall (directly or indirectly) receive the signals $(4.2,6.2)$ from the nervous system's processes that detect the current situation (including the current controlled processes of interaction) as well as the state and the probability of TEF achievement in this situation. However, the nervous system's processes that detect these controlled processes of interaction generate only signals that reflect the accuracy of determination of these controlled processes of interaction (which can be influenced by the corresponding processes of influence) without taking into account other processes of the current situation and the probability of satisfaction of TEFs, i.e. without receiving the corresponding signals. Therefore, the nervous system's processes that are the generators shall receive the main influences not directly from the specified detectors of the controlled processes of interaction, but indirectly, through the nervous system's processes that will generate these main incoming signals to the generators of the nervous system (hereinafter referred to as the primary generators). The signal of these primary generators, according to the above, shall also depend on the signals of detectors that detect the processes that form the current situation and detectors of the state and probability of TEF achievement (4.3).

Thus, the primary generators shall receive the activating (6.2.10) influences from the detectors that detect the current controlled processes of interaction and also, directly or indirectly, the influences from the nervous system's processes that detect other existing processes of the universe, their properties and the values of characteristics (i.e. determine the current situation) and from the nervous system's processes that detect the current state and probability of TEF achievement.

\subsubsection{Controlled Deactivation of Nervous System's Processes (Primary Generators) that Generate Main Signals to Generators}

The controlled processes of interaction are a part (subprocesses) of other (hierarchically higher) processes (3.1) that are formed by the interaction of the current set of interrelated processes of the universe with a certain set of properties and values of characteristics and are the current situation (4.1.2, 3.1) in which these controlled processes of interaction are implemented. Moreover, one and the same controlled processes of interaction can, at different time (at different stages of interaction of the organism's processes with other processes of the universe), be the subprocesses of different hierarchically higher processes (3.1) - implemented in different current situations. Therefore, different primary generators receiving the influences from the same detectors of controlled processes of interaction (7.3.1) can generate one and he same outcoming signals in different current (current-foreseeable - 4.1.2) situations. However, in order to minimize the consumption of resources (5.3.6) in each current (currentforeseeable - 4.1.2) situation, the generators shall generate only such sequences of signal combinations that will lead to the processes of influences that will ensure achievement of TEFs of the organism in this situation (7.2.1). The signals generated by generators are caused and determined by the signals generated by the primary generators, which, in their turn, are caused and determined by the signals dependent on the detectors of current and foreseeable processes of the universe (the sets of processes, sets of interrelationships and properties, and values of characteristics of these processes) that can influence the achievement of TEFs of the organism (7.3.1). Therefore, in each current situation, the signals about the accuracy of determination of the processes shall be generated only by those primary generators (of those receiving in this situation the activating signals from the detectors of controlled processes of interaction) and with such values of these signals the influences of which will lead to the generation by the generators necessary for achievement of the TEFs in this current situation signals about the accuracy of determination of the corresponding processes of influence. Thus, the primary generators receiving the activating influences from the detectors of controlled processes of interaction shall also receive the permanent deactivating signals (6.2.10), and the influence of these deactivating signals shall depend on the signals of detectors of current and foreseeable processes that form the current (current-foreseeable 4.1.2) situation, their properties and values of characteristics.

The implementation of the deactivating influence on the primary generators shall be carried out by the 
group of the nervous system's processes (hereinafter referred to as the deactivators of primary generators) that directly influence (interrelated with them 6.2.3) these primary generators. These nervous system's processes (deactivators of primary generators) shall constantly generate the signals (in the absence of the external activating influences, unlike other nervous system's processes, are self-activated with a certain periodicity) that will deactivate the primary generators activated by the signals of the detectors of controlled processes of interaction. However, in order to enable for certain primary generators the possibility to generate signals that will lead to the generation by the generators of the signals required to achieve the TEFs of the organism in certain current situations, these deactivators of primary generators, in their turn, shall be deactivated by other processes of the nervous system (hereinafter referred to as the unblocking deactivators). Therefore, the unblocking deactivators shall generate the signals when detecting certain current situations, and, thus, shall receive the activating influences from nervous system's processes that detect the processes (their properties and values of characteristics) that form this current (current-foreseeable - 4.1.2) situation.

The interrelationships between the described processes of the nervous system shall be adjusted according to the general rules for the processes of the nervous system $(6.2,6.3)$.

\subsubsection{Modulation of Interrelation of Nervous System's Processes that Deactivate Deactivators of Primary Generators with Detectors of Processes that Form Current Situation by Signals from Detectors of State and Probability of TEF Achievement}

The relations between the processes of the nervous system are formed randomly (6.2.14), with further strengthening or weakening $(6.2 .12,6.2 .13)$, based on the statistical selection of the detected process (6.2.18) and under the possible influence of the modulating influences from other processes of the nervous system (6.2.10). The deactivators of primary generators receive the deactivating signals from unblocking deactivators, which receive the activating influences from the nervous system's processes that detect the processes (their properties and values of characteristics) that form a certain current situation (7.3.2). These deactivating signals (according to 7.2.1, 7.3.1, 7.3.2) shall, in their turn, be such that in this detected current situation the influences caused by them will eventually lead to such processes of influence that will ensure the required for the increase of the state and probability of achievement of TEFs of the organism interaction of the controlled processes of interaction and other processes of the universe. Therefore, the nervous system's processes that generate these deactivating signals shall have such interrelationships with the processes influencing them (6.2) that, when detecting the process by them, they generate these deactivating signals (with the required characteristics) necessary to increase the probability of achievement of TEFs. Thus, to ensure the generation of the required deactivating signals in future interactions in the situations similar to the current one (4.1.1), it is necessary that the statistical selection of the processes detected by the unblocking deactivators depends on the state and probability of TEF achievement in this current situation. I.e. those relations through which the unblocking deactivators receive the signals whose influences lead to the detection of such processes by them the signals about the accuracy of determination of which lead to the increase in the state and probability of achievement of TEFs in a certain current situation shall have more possibilities to be strengthened (or not weakened) than those relations signals through which do not lead to the increase in the state and probability of TEF achievement in this current situation (4.1.2). As the detection of the state and increase of the probability of TEF achievement in each current situation is carried out by the set of the nervous system's processes that are the detectors of the state and probability of TEF achievement (4.3), maintaining unchanged and/or strengthening the relations between the unblocking deactivators and the nervous system's processes sending the signals to them shall depend on the signals of the detectors of state and probability of TEF achievement, i.e. shall be modulated (6.2.10) by these signals (through a direct or indirect interaction 4.2.1), thus, the statistical selection (6.2.18) of the processes detected by the unblocking deactivators will be modulating. Therefore, in the future, when detecting the current situation similar to the one in which, in the past, such influences were exerted on the unblocking deactivators that the generated by them signals about the accuracy of determination of the processes detected by them eventually led to achievement of TEFs (to generation of signals by the detectors of state and probability of TEF achievement), these unblocking deactivators are very likely to detect the same processes and, therefore, generate the same signals and, thus, the organism's TEFs are very likely to be achieved again (4.1.1). 
The modulating signals from the detectors of state and probability of TEF achievement will change (6.2.10) from 0 (no modulating influence) to a certain maximal value (the maximal possible modulating influence that these detectors of state and probability of TEF achievement can exert on a certain group of the processes of the nervous system with which they are interrelated).

\subsubsection{Relevant Interrelation of Nervous System's Processes that are Included to System of Formation of Main Signals that Influence Formation of Signal by Generators}

Each process of the nervous system for self-adjustment to the detection of a certain process shall be interrelated with other processes that can form relations with this process through which the influences on it will be exerted and the signals from which shall form the interrelated sequences of combinations (6.2.18). Therefore, every primary generator shall receive the influences (be interrelated with them 6.2.3, 6.2.20) from such detectors of controlled processes of interaction and the detectors of current situation and the detectors of state and probability of TEF achievement (through the chain of deactivators of primary generators and unblocking deactivators - 7.3.2, 7.3.3) whose signals shall be interrelated and form the interrelated sequences of signal combinations.

The term "relevant nervous system's processes" can be used hereinafter to define the nervous system's processes that influence a certain process of the nervous system, generate the interrelated signals (detect the interrelated processes and/or their properties and the values of characteristics) and lead to the adjustment of this process of the nervous system to detection of certain processes of the universe.

\subsubsection{Generators as Detectors of the Process that Form Current Situation}

The nervous system's processes that are the generators detect the processes of influence (7.2.2) that are caused and determined (controlled) by the signals of these generators and influence the controlled processes of interaction (7.3.1) that, in their turn, are the subprocesses of a certain process forming a certain current situation (7.3.1, 7.3.2, 4.1.2). Therefore, every process of influence is a part of the processes that form the current situation (the set of interrelated processes with certain properties and values of characteristics that, interacting with one another, can, to a different extent, directly or indirectly, influence achievement of TEFs of the organism - 4.1.2). Thus, each generator is simultaneously the detector of a part of the processes (the processes of influence) that form this current (current-foreseeable) situation.

The primary generator receiving the activating influences from the detectors of a certain controlled process of interaction shall also receive the indirect influences from the corresponding, relevant (7.3.4) detectors of processes that form the current situation (7.3.1, 7.3.2). Thus, every primary generator, in addition to the indirect influences from other nervous system's processes that detect the current situation, shall receive the indirect influences from the corresponding, relevant generators as from the detectors of the processes that form the current situation.

\subsubsection{Generators as Detectors of the Process that are the Subprocesses of Controlled Processes of Interactions}

The nervous system's processes that are the generators detect the processes of influence (7.2.2) that are caused and determined (controlled) by the signals of these generators and influence the controlled processes of interaction (7.3.1). Controlled processes of interaction are processes of interaction of the processes of the organism with one another or with other processes of the universe the course of which the processes of influence can influence (7.3.1). Each process of influence or sequence of combinations of processes of influence shall be implemented only if a certain (the one that can be influenced by these processes of influence) process of interaction of organism's processes with one another or with other processes of the universe is carried out. Therefore, each nervous system's process that is the generator indirectly (through detecting a certain processes of influence) detects a certain controlled process of interaction, together with which process of influence detected by this process of nervous system (which is the generator) is implemented. 


\subsubsection{Hierarchy of Processes of Influence and Generators and Cascading of System of Formation of Main Signals that Influence Generation of Signal by Generators}

According to the initial assumption (3.1), the universe is a set of hierarchical processes, and the processes in the universe, including a living organism itself (3.2), are a certain combination of other, hierarchically lower processes, except for the processes of the initial level of the hierarchy. The processes of the nervous system interact with the processes of the universe exerting on them the sequences of combinations of influences (4.2.3) that lead to the implementation of the processes of influence required for increase of the probability of achievement of TEFs (7.2.2). In the sequences of combinations of influences and, thus, in the corresponding processes of influence, the hierarchy of the processes of the universe (and their properties) with which the processes of the nervous system interact $(5.4 .2,5.4 .7)$ is reflected. The specified sequences of combinations of influences are generated by the hierarchical blocks of the processes of the nervous system - hierarchical blocks of generators $(5.4 .2,4.3)$.

The primary generators receiving the activating influences from the detectors of a certain controlled process of interaction shall also receive the indirect influences from the corresponding, relevant (7.3.4) detectors of processes that form the current situation and the relevant detectors of state and probability of TEF achievement (7.3.1, 7.3.2). Thus, in view of the above, the primary generators that generate the signals to the generators of a certain level of the hierarchy shall receive the influences from the corresponding, relevant for that level of the hierarchy, detectors of controlled processes of interaction, detectors of the processes of the universe that form the current situation, and the detectors of state and probability of TEF achievement.

The generators of higher levels of the hierarchy detect the sequences of combinations of processes of influence of higher levels of the hierarchy — the processes of influence of higher level of the hierarchy (7.2.2, 5.4.2). As the processes of influence are the processes that are a part of the current situation (7.3.5), and the processes of influence of the lower level of the hierarchy are the subprocesses of a certain process of influence of the higher level of the hierarchy, the process of influence of the higher level of the hierarchy is a part of the current situation for the corresponding process of influence of the lower level of the hierarchy that is a part of this hierarchically higher process. Therefore, the primary generators that generate the signals to the generators of a certain level of the hierarchy shall receive the indirect influences from the corresponding generators of the higher level of the hierarchy or from the primary generators influencing them as from the detectors of processes of the current situation whose part the processes of influence generated by these generators are.

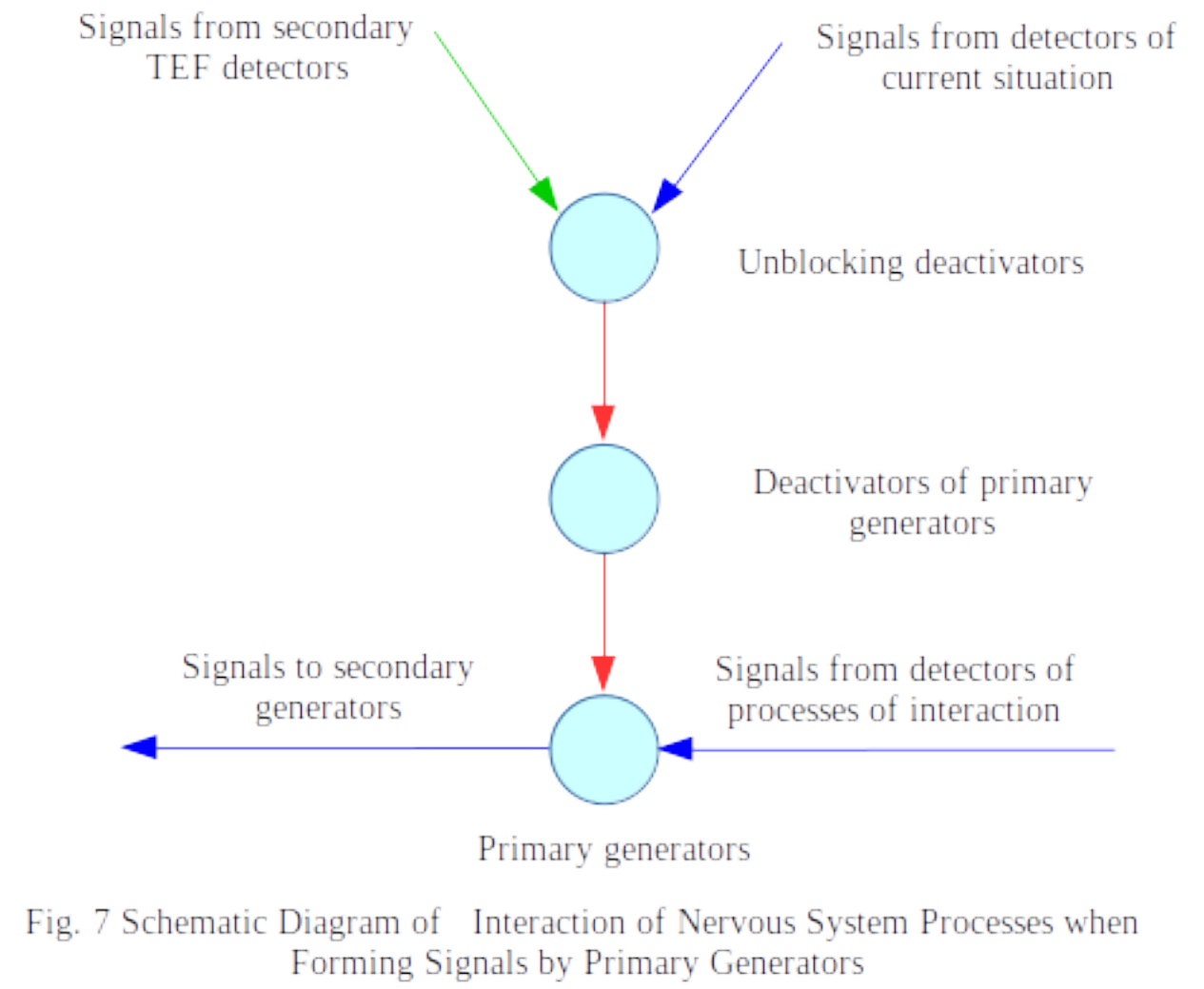




\subsection{Principles of Construction and Functioning of Control System of State and Probability of TEF Achievement}

\subsubsection{Formation of Interrelationships that Transmit Influence to Unblocking Deactivators under Influence of Integrated Signal from Relevant Detectors of State and Probability of TEF Achievement}

The organism's nervous system includes the processes that detect the processes that reflect the state and probability of achievement of TEFs of the organism (4.3). The signals generated by these processes of the nervous system exert the modulating influences (through direct or indirect interaction) on the nervous system's processes that are included to the system of formation of main signals (7.3) that influence the generators during their adjustment and detection of the processes of influence (7.3.3).

The more complicated the organism (the greater the number of subprocesses that are a part of the process that is this organism) is and the more complicated (the greater the number of the interacting processes and their properties and interdependencies that this organism perceives or can perceive and that influences or can influence when achieving the TEFs, and the greater the number of future interactions that it takes into account - 4.1.1) its strategy of achieving TEFs is, the more varied (the greater the number of possible interactions of different processes in different sequences and combinations of interactions) can be the ways to achieve the TEFs (with the same or different degree of TEF achievement in case of each of the possible ways) of this organism in a certain situation. Therefore, the greater the number of interacting processes can determine or influence the probability of achievement of TEFs of the organism in this situation. Thus, in order to ensure the selection of the optimal way to achieve TEFs of the organism in a certain situation, the greater number of such processes shall be detected by the detectors of state and probability of TEF achievement that are part of this organism and, therefore, the greater the number of the processes shall reflect the existing state and probability of achievement of TEFs of the organism in this situation.

As specified above, the nervous system of the organism shall ensure the efficient and effective achievement of TEFs of the organism (reduction of the number of unachieved TEFs and increase of the number of achieved TEFs and/or increase of their degree of achievement in the current and future, after a certain number of cycles of interactions of processes, situations - 4.1.2, 4.1.3), i.e. choose the way to achieve the TEFs in a certain situation that will ensure the most efficient and effective achievement of TEFs of the organism in the current (current-foreseeable - 4.1.2) situation. Therefore, to ensure generating by the generators, in a certain situation, such sequences of signal combinations that will lead to the implementation of the most efficient and effective way to achieve the TEFs in this situation, it is necessary for each generator to take into account the signals from all relevant (7.3.4) for it detectors of state and probability of TEF achievement that determine the probable state and probability of achievement of TEFs in case of various ways of achieving TEFs that can be implemented by the organism in this situation. Therefore, the modulating influences from the detectors of state and probability of TEF achievement (through a direct or indirect interaction) on the nervous system's processes that are included to the system of formation of the main signals that influence the adjustment of the generator to the detection of certain processes (7.3.3) shall come to these processes of the nervous system from all relevant (7.3.4) detectors of state and probability of TEF achievement. In this case, the formation of interrelationships of these processes (7.3.3) shall be carried out under the influence of the integrated modulating influence of all relevant detectors of state and probability of TEF achievement.

\subsubsection{Formation of New Detectors of State and Probability of TEF Achievement in Nervous System of Combined Type}

During the development of the organism and its interaction with the external processes, the processes of the nervous system (the self-adjusting part of the processes in the nervous system of the combined type - 4.3) of the organism change to detect and generate new sequences of combinations of incoming and outcoming signals $(4.1 .3,4.3)$ corresponding to new/changed processes of the universe of various levels 
of the hierarchy (5.4.2, 7.2.9) with which the processes of the nervous system (4.2) interact during the implementation of the strategy of achieving TEFs of the organism (5.2.2). As each sequence of combinations of outcoming signals shall contribute to achievement of TEFs $(3.2,4.1 .3)$, in order to ensure the formation of new generators of sequences of control signal combinations based on the signals from the detectors of new sequences of combinations of incoming signals, it is necessary to determine how much, in a certain detected situation (detected and generated combinations of signal sequences), the probability of achievement of TEFs of the organism increases. I.e. to what degree these outcoming sequences of signal combinations generated by the generator lead to such a sequence of combinations of interactions (4.2.3) of the organism's processes, including the processes of the nervous system of the organism, and the other processes of the universe, that will leads to satisfaction of the organism's TEFs in a new detected situation. Therefore, the structure and properties of the nervous system of the combined type shall provide rules of formation and modification of blocks of detectors of state and probability of TEF achievement that could detect the state and probability of achievement of TEFs in new situations and the participation of these detectors in the formation of new generators of sequences of combinations of outcoming signals together with the preprogrammed detectors of the TEF achievement (5.2.5).

\subsubsection{Sequence of Formation of New Detectors of State and Probability of TEF Achievement}

In the nervous system of the combined type (4.3), the set of detectors of state and probability of TEF achievement can either consist entirely of the preprogrammed detectors or include such detectors in combination with the self-adjusting detectors of state and probability of TEF achievement (5.2.5, 7.4.2). In this case, the self-adjusting detectors of state and probability of TEF achievement shall adjust themselves to detect such processes that are interrelated with the processes detected by the preprogrammed detectors of state and probability of TEF achievement and at implementation of which the probability of implementation of these processes (second) is increased (5.2.5). Thus, the adjustment of self-adjusting detectors of state and probability of TEF achievement (formation, maintenance of stability and breaking of the interrelationships with the nervous system's processes that will influence them - 6.2) to the detection of the processes shall depend (shall be modulated [6.2.10] by the signals generated by the corresponding processes of the nervous system) on the processes detected by the preprogrammed detectors of state and probability of TEF achievement or on the processes detected by the self-adjusting detectors of state and probability of TEF achievement that are already adjusted to the detection of the processes according to the same rule. Therefore, the self-adjusting detectors of state and probability of TEF achievement in the nervous system of the combined type will adjust themselves to the detection of the processes sequentially. The first to adjust are self-adjusting detectors that adjust themselves to the detection of the processes that are interrelated with the processes that are directly detected by the preprogrammed detectors of state and probability of TEF achievement (included with them to a certain process of a higher level of the hierarchy or is for them the process of a higher level - 3.1). The following self-adjusting detectors of state and probability of TEF achievement will adjust themselves to the detection of the processes that can be interrelated with the processes detected by the preprogrammed detectors as well as with the processes detected by already adjusted self-adjusting detectors of state and probability of TEF achievement. If the interrelationship between the processes detected by the detectors of state and probability of TEF achievement is changed (weakens or disappears as a result of the changes of the processes, with which the processes of the nervous system interact, or the changes in the detection of the interdependencies between these processes by the nervous system - 6.2.5), then, therefore, the modulating influence on the interrelationships through which the signals are transmitted from the processes that influence the self-adjusting detectors of state and probability of TEF achievement from the corresponding detector of state and probability of TEF achievement, under the influence of the modulating influences of which these interrelationships were formed and maintained, will change (weaken). The reduction of this influence will lead to readjestment of the specified detector to the generation of a smaller signal or to detection of another process depending on the modulating influences coming to it from the specified and other detectors of state and probability of TEF achievement and depending on the interrelationships that it can create with the processes influencing it (6.2). Such a readjustment will lead, in its turn, to the appropriate readjustment of those detectors of state and 
probability of TEF achievement that were formed under the influence of the modulating signals from this readjusted detector.

\subsubsection{Formation of Interrelationships through which Influence is Transmitted on Self- adjusting Detectors of State and Probability of TEF Achievement under Influence of Integrated Signal from Relevant Detectors of State and Probability of TEF Achievement}

As specified above (7.4.1), the more complicated the organism is and the more complicated its strategy of achieving TEFs is, the more varied the ways of achieving TEFs by this organism can be. Therefore, with the greater number of other processes that are detected by the detectors of the state and probability of TEF achievement the process can be interrelated that is detected by a certain detector of the state and probability of TEF achievement in different situations. Thus, the formation of the interrelationships influencing on this certain self-adjusting detector of state and probability of TEF achievement will be implemented under the influence of the signals from different detectors of state and probability of TEF achievement that detect the processes interrelated with the process detected by this detector. I.e. every self-adjusting detector of state and probability of TEF achievement will form the interrelationships with other nervous system's processes that will influence it under the action of the integrated signal from the corresponding preprogrammed or already adjusted self-adjusting detectors of state and probability of TEF achievement.

\subsubsection{Structural Hierarchy of Detectors of State and Probability of TEF Achievement}

To achieve the TEFs, the generators of the nervous system generate the sequences of combinations of signals $(4.2 .3,4.3)$, which can consist of a combination of (in parallel generated) sequences of signals generated by different generators (6.1.1). Therefore, the same detectors of state and probability of TEF achievement can be relevant (7.3.4) and, thus, can be interrelated (3.1) with different nervous system's processes whose relations are modulated depending on the signals of these detectors of state and probability of TEF achievement. Also, the signals from these detectors of state and probability of TEF achievement shall influence the formation of new detectors of state and probability of TEF achievement (7.4.3). I.e., in the nervous system, it is necessary to provide the parallel transmission (duplication of transmission) of the same integrated $(7.4 .1,7.4 .4)$ signals generated by the detectors of state and probability of TEF achievement to different nervous system's processes whose relations are modulated depending on the signals of these detectors. Taking into account the structure of the minimal uniform process of the nervous system (6.3) underlying the hierarchically higher processes of the nervous system (6.1.2), the nervous system with a two-level structure of the detectors of state and probability of TEF achievement will be more resource-efficient thanks to the reduction of duplication of the processes of transmission (6.3.4) of the modulating signals. At the first level of this structure, the nervous system's processes will function that directly detect the processes that lead to and/or are realized (are implemented) when the state and/or the probability of achievement of TEFs is increasing or decreasing (7.4.1) - the primary detectors of state and probability of TEF achievement. At the second level of this structure, the nervous system's processes will function that will perceive the influences from the primary detectors of state and probability of TEF achievement and will generate the signals that will have the modulating influence on the formation of the interrelationships influencing the unblocking deactivators (7.3.3) and the self-adjusting primary detectors of state and probability of TEF achievement (7.4.3) — the secondary detectors of state and probability of TEF achievement. Moreover, the secondary detectors of state and probability of TEF achievement will detect the integrated, hierarchically higher than those detected by the primary detectors of state and probability of TEF achievement $(3.1,5.4)$, processes. The modulating signals generated by the secondary detectors of state and probability of TEF achievement change their values from 0 to 1 (6.2.10).

\subsubsection{Activating and Deactivating Influences of Primary Detectors of State and Probability of TEF Achievement on Secondary Detectors of State and Probability of TEF Achievement}

As specified above (6.2.11), both the activating and deactivating signals from other processes of the nervous system lead to an increase in the accuracy of determination of the processes of the universe by the 
processes of the nervous system. Besides, the system responsible for evaluating the state and probability of achievement of TEFs identifies the processes that reflect the parameters of the target evolution functions known to the system that are dissatisfied/satisfied in the current situation or can be with a certain probability dissatisfied/satisfied in the foreseeable future situation and the level of their dissatisfaction/satisfaction $(4.1,4.3)$. Therefore, the processes of the nervous system, when determining the state or the probability of achievement of TEFs, in addition to the processes that are implemented when the state and the probability of achievement of TEFs is increasing, shall also detect the processes that are implemented when the state and the probability of achievement of TEFs is reducing.

The system of control of the state and the probability of TEF achievement has two levels of the hierarchy consisting of the primary and the secondary detectors of state and probability of TEF achievement (7.4.5). Moreover, the primary detectors of state and probability of TEF achievement detect the processes that lead to and/or are realized (are implemented) when the state and/or the probability of achievement of TEFs is increasing or decreasing, and the secondary detectors under the influence of the signals from the primary detectors detect the integrated, hierarchically higher than those detected by the primary detectors of state and probability of TEF achievement, processes and generate the signals that exert the modulating influences for adjusting of the relations of other processes of the nervous system. Thus, the primary detectors that detect the processes that are implemented when the state and the probability of achievement of TEFs is increasing shall exert the activating influences on the secondary detectors of state and probability of TEF achievement, and the primary detectors that detect the processes that are implemented when the state and the probability of achievement of TEFs is decreasing- the deactivating ones.

According to the above (in the current section and in subsections 7.4.5, 7.4.3, 5.2.5), in the nervous system of the combined type, the new self-adjusting primary detectors of state and probability of TEF achievement, whose relations (through which they receive the influences from other processes of the nervous system) and, thus, their adjustment to detecting of certain processes, are formed under the influence of the modulating signals from the secondary detectors of state and probability of TEF achievement, detect the processes that are implemented when the state and the probability of achievement of TEFs is increasing (5.2.5, 7.4.3). Therefore, these self-adjusting primary detectors exert the activating influences on the secondary detectors of state and probability of TEF achievement that leads to the generation of the modulating signals by the secondary detectors of state and probability of TEF achievement under the influence of which the generators adjust themselves to the generation of the sequences of combinations of signals that lead to the satisfaction of the organism's TEFs (7.4.5, 7.3.3).

Thus, the deactivating influences received by the secondary detectors of state and probability of TEF achievement from the primary detectors shall be received from the preprogrammed primary detectors of state and probability of TEF achievement (5.2.5). Moreover, these primary detectors themselves can receive influences from both the preprogrammed processes of the nervous system and the self-adjusting detectors of the processes (4.3), which can adjust themselves to the detection of the processes interrelated with the processes detected by these preprogrammed primary detectors of state and probability of TEF achievement (5.2, 5.4.1).

\subsubsection{Generators as Detectors of Processes whose Implementation Can Lead to Increase in State and/or Probability of Achievement of TEFs (Self-adjusting Primary Detectors of State and Probability of TEF Achievement)}

Each generator is a detector of the process of influence (7.2.2) of a certain level of the hierarchy (5.4.2, 7.3.7). Each process of influence is a process during the implementation of which in a certain current situation such a change in the characteristics of the controlled processes of interaction takes place that increases the probability of achievement of TEFs of the organism in this situation. Therefore, the processes of the nervous system of the organism belonging to the blocks of synthesis of sequences of combinations of signals (generators) are also the detectors of the processes during the implementation of which in this situation the probability of achievement of TEFs of the organism increases. Thus, the relevant (7.3.4) nervous system's processes that are the generators shall form the interrelationships and influence certain groups of self-adjusting primary detectors of state and probability of TEF achievement that will detect the processes that lead to and/or are realized when the state and/or the probability of 
achievement of TEFs is increasing when these generators generate the signals about the accuracy of the process determination.

\subsubsection{Sequence of Activation (Generation of Signals About Accuracy of Determination of Processes) of Primary Detectors of State and Probability of TEF Achievement when Generating Sequence of Signal Combinations}

The nervous system of the organism implements the strategy (5.2.2) of achieving TEFs of the organism by generating by its corresponding processes (generators - 4.3) the sequences of signal combinations that lead to the necessary flow of the processes of the universe whose part this nervous system is (4.2.3). When implementing the changes in the processes of the universe forming a certain current situation (4.1.2), this current situation changes under the influence of each element of the signal of the generated by the generators sequence of signal combinations and the interaction of the processes of the universe under the action of other factors (3.1). These changes in the current situation are detected by the processes of the nervous system as a new current-foreseeable (4.1.2) situation. In this case (when changing the currentforeseeable situation), the processes detected by the primary detectors of state and the probability of TEF achievement can change and, therefore, the generated by these detectors signals about the accuracy of determination of the processes detected by them can change. I.e., when the current-foreseeable situation detected by processes of the nervous system changes as a result of interaction among the processes of the universe that form this situation, including the generators of the nervous system, the signals generated by the primary detectors of state and probability of TEF achievement will be being changed. Thus, due to the sequential change of the current-foreseeable situation under the influence of the sequences of signal combinations generated by the generators of the nervous system and other factors resulting in the change of the current situation, the activation of these primary detectors (generation of signals about the accuracy of determination of the processes by them) will form a certain sequence of combinations of simultaneously (in a certain current-foreseeable situation) activated (those generating the signals) primary detectors of state and probability of TEF achievement. This sequence of combinations of operating (generating the signals) primary detectors of state and probability of TEF achievement will be formed as a result of adjustment of the specified detectors to the detection of the processes according to the rules described above $(7.4 .3,6.2)$. Therefore, the described sequence of combinations will lead to such changes in the sequences of combinations of modulating signals that influence the adjustment of the generators to the detection of the processes $(4.1 .3,4.2 .3,7.3 .3)$, that the sequences of signal combinations that will eventually be generated by the generators will lead to the satisfaction of the organism's TEFs (4.1.3), i.e. to the activation (generation of the signals) of the preprogrammed detectors of state and probability of TEF achievement (7.4.3).

\subsubsection{Correlation between Sequences of Signal Combinations Generated by Generators and Primary Detectors of State and Probability of TEF Achievement}

The sequences of signal combinations generated in a certain current-foreseeable situation by the generators shall lead to the increase in the probability of achievement of TEFs of the organism in this current-foreseeable situation (4.1.3). The adjustment of generators to the detection of certain processes and the corresponding generation of sequences of signal combinations (6.1.1) is carried out by them by self-adjustment to the detection of the interrelated signals from the processes of the nervous system (primary generators) whose signal (adjustment to the detection of a certain process by them) depends on the modulating influence of the relevant processes that detect the state and probability of achievement of TEFs of the organism (7.2.5, 7.2.6, 7.3). Therefore, the ensuring of adjustment, change and consistent (6.2.6) generation of the sequences of signal combinations required to achieve the TEFs will be carried out by the nervous system's generators under the action of the sequences of combinations of modulating influences relevant to these generators from the secondary detectors of state and probability of TEF achievement (7.4.5). These modulating influences, in their turn, will form under the influence of the sequences of signal combinations generated by the primary detectors of state and probability of TEF achievement that will generate these signals in a certain sequence and combination (6.1.1) that will form as a result of the change in the detected current-foreseeable (7.4.8) situation. 


\subsubsection{Inverse Deactivating Influences on Processes of Control of Probability of TEF Achievement}

Under the action of the integrated modulating signals generated by the secondary detectors of state and probability of TEF achievement, the primary detectors of state and probability of TEF achievement and the unblocking deactivators relevant to them adjust themselves to the detection of certain processes (7.4.1, 7.4.3, 7.4.5). Moreover, the signals themselves generated by the secondary detectors of state and probability of TEF achievement are generated as a result of the activating influences exerted on them, including influences from the self-adjusting primary detectors of state and probability of TEF achievement (7.4.5, 5.2.5). I.e. between the primary self-adjusting and secondary detectors of state and probability of TEF achievement there is a certain positive interrelationship (positive feedback) through which the uncontrolled mutual strengthening of the influence of these processes on each other is possible. In order to eliminate the negative influence of the described positive interrelationship on the self-adjusting primary detectors of state and probability of TEF achievement, the secondary detectors, in addition to the activating signals from the primary detectors, shall also receive the deactivating signals from other processes of the nervous system (receive negative feedback that will compensate the negative influence of the positive feedback). Moreover, in order to implement the possibility of adjustment and maintaining the stability $(6.2 .4,6.2 .5,6.2 .6)$ of the adjusted primary detectors of state and probability of TEF achievement (mutually balanced increase and decrease of the signals) depending on the modulating signals of the secondary detectors (7.4.5), these deactivating signals shall have the same character of dependence on the signals of the secondary detectors of state and probability of TEF achievement as the signals of the corresponding primary detectors of state and probability of TEF achievement. I.e. the interrelationships between the nervous system's processes that will generate the specified deactivating signals and the nervous system's processes that will transmit their influences (signals) on them through these interrelationships shall, as for the primary detectors of state and probability of TEF achievement (7.4.3, 7.4.4, 7.4.5), be modulated by the integrated signals from the relevant secondary detectors of state and probability of TEF achievement.

The primary detectors of state and probability of TEF achievement detect the processes during the implementation of which the probability of achievement of TEFs of the organism increases/decreases (7.4.5, 7.4.6). Moreover, the processes themselves detected by the specified primary detectors can, at different time (at different stages of interaction of the organism's processes with other processes of the universe), be the subprocesses of different hierarchically higher processes (3.1), i.e. implemented in different current (current-foreseeable - 4.1.2) situations. And in all these situations, under the action of the modulating signals from the secondary detectors activated by the primary detectors of state and probability of TEF achievement, both the self-adjusting primary detectors relevant to them and the relevant unblocking deactivators shall form $(7.4 .3,7.3 .3)$ that will lead to the adjustment of generators to the detection of such processes of influence the implementation of which will increase probability of achievement of TEFs of the organism in the specified current-foreseeable situations. I.e. the self-adjusting primary detectors of state and probability of TEF achievement and the unblocking deactivators shall receive the same relevant modulating influences from the secondary detectors of state and probability of TEF achievement in the same current situations. In order to ensure the increase in the state and the probability of achievement of TEFs of the organism in each situation, it is necessary to provide the adjustment and maintaining the stability $(6.2 .4,6.2 .5,6.2 .6)$ of the corresponding unblocking deactivators that are subject to the modulating influences from the specified secondary detectors. As the nervous system's processes that generate the specified deactivating signals to the secondary detectors will decrease (6.2.10) the modulating influence generated by these secondary detectors both on the self-adjusting primary detectors and on the unblocking deactivators, to ensure the formation and maintenance of the stability of the unblocking deactivators the nervous system's processes that will generate the deactivating influences on the secondary detectors of state and probability of TEF achievement shall adjust themselves in the same way as the unblocking deactivators, have the same properties (3.1) that will allow them to adjust themselves (6.2) to the detection of the same processes, and the same interrelation (5.4.1, 7.3.2) with the nervous system's processes that detect the current situation and can establish the interrelationships with them. To minimize the use of the organism's resources by avoiding unnecessary consumption of resources when transmitting the same signals from the detectors of the process that form 
the current situation to the non-interrelated processes of the nervous system, the nervous system's processes that provide the generation of the deactivating signals to the secondary detectors of state and probability of TEF achievement shall be interrelated with the unblocking deactivators (5.4.1). I.e. these processes shall form together with them such a structure of the nervous system's processes that will ensure their maximal interrelation with one another and the same interrelation with the processes that will transmit the influences to them - the processes that are the unblocking deactivators placed alternately (one next to the other) with the processes that generate the deactivating signals to the secondary detectors of state and probability of TEF achievement.

\subsection{System of Optimization of Implementation of Controlled Processes of Interaction (Smoothing)}

\subsubsection{Overload and Overregulation when Implementing Controlled Processes of Interaction}

The more complicated the organism (the greater the number of subprocesses that are included to the process that is this organism), the greater the probability of the need and/or possibility of simultaneous influence of several organism's processes on the group of interrelated processes (or a certain process) of the universe, including on other processes of the organism, to change their characteristics (4.2) in order to increase the state and/or the probability of achievement of TEFs of the organism in the current situation (4.1). This influence is carried out either directly by the processes of the nervous system - the generators, or by other processes of the organism under the influence of the sequences of combinations of signals generated by these generators $(4.2,4.3,7.2 .1)$. The required value of change in the characteristics of the specified group of the processes (or the process) of the universe being influenced can be achieved, in this case, by different combinations of characteristics of sequences of combinations of signals generated by the generators:

- a small group of generators influences a small group of processes by changing their certain characteristics to a significant value, or all the necessary and possible relevant generators influence all the necessary and possible (for change) processes of the universe by changing their certain characteristics to a relatively small value;

- an abrupt change in the characteristics of the processes influenced by the sequences of combinations of signals generated by the generators, or a gradual foresighted change in these characteristics.

When implementing changes in the characteristics of the processes of the universe under the influence of sequences of combinations of signals generated by a small group of generators, with a significant change in the value of the characteristics of a small number of processes of the universe that they influence, there may be an overload of the organism's processes involved, i.e. such a change in the characteristics of these processes that can lead to their premature destruction.

When implementing the changes in the characteristics of the processes of the universe under the influence of the sequences of signal combinations that have such characteristics that lead to highly abrupt (faster than necessary and sufficient to achieve TEFs of the organism in the current situation) changes in the characteristics of these processes of the universe, the overregulation is possible when changing their desired characteristics (greater than necessary to achieve TEFs of the organism, change of these characteristics with the subsequent need for corrective influence on them).

7.5.2. Inability of Guaranteed Adjustment of Generators to Generation of Sequences of Signal Combinations that will not Lead to Overload and Overregulation under Action of Modulating Signals from Detectors of State and Probability of TEF Achievement

The generators adjust themselves to the generation of the sequences of signal combinations that lead to the implementation of certain processes of influence (7.2.2) under the action of the modulating signals from the detectors of state and the probability of TEF achievement (4.3) according to the principles described above $(7.3,7.4)$. The overload and overregulation, that can occur when achieving the organism's TEFs under the influence of the signals generated by the generators, do not lead to the current 
impossibility of achievement of TEFs of the organism (7.5.1). Therefore, it will be impossible to guarantee adjustment of the generators that lead to the implementation of the specified processes of influence, according to the rules described above $(7.3,7.4)$, to generation of such sequences of signal combinations that will not lead to the overload and/or overregulation.

\subsubsection{Basic Rules of Functioning and Principles of Construction of Nervous System's Processes that Help to Avoid Overload and Overregulation when Implementing Controlled Processes of Interaction}

The generators adjust themselves to the generation of the sequences of signal combinations under the action of the main signals generated by the primary generators (7.3.1). The primary generators, in their turn, generate the sequences of signal combinations receiving the activating influences from the processes that detect the current controlled processes of interaction (7.3.1). The primary generators adjust themselves to the detection of the processes under the indirect influence of the modulating signals from the detectors of state and probability of TEF achievement (7.3). For controlled processes of interaction, it is impossible to guarantee adjustment (under the influence of the modulating signals from the detectors of state and probability of TEF achievement) of the generators to the generation of the sequences of signal combinations that will ensure the absence of the overload and overregulation (7.5.2). Therefore, this adjustment of the specified controlled processes of interaction shall occur earlier, before the influence on the primary generators from the detectors detecting these processes. I.e. the activating influences (the sequences of signal combinations) coming to the primary generators shall come not directly from the nervous system's processes that detect the specified controlled processes of interaction, but indirectly through the nervous system's processes that will change these sequences of signal combinations according to the rules that will lead to avoidance of the overload and overregulation. Taking into account causes that can lead to the overload and overregulation (the activation of smaller than necessary group of generators and/or the generation by them of sequences of signal combinations with an abrupt change in the characteristics - 7.5.1), these rules shall be as follows:

- To avoid the overload due to the activation of a small enough group of generators in a certain situation, it is necessary to implement the possibility of co-activation of the relevant nervous system's processes that will be influenced by the processes that detect the controlled processes of interaction and influence the primary generators. Such a co-activation will allow to exert the activating influence on the most of the relevant primary generators and, therefore, increase the group of simultaneously activated generators and distribute the load among them and among the processes that they influence through the secondary generators;

- To avoid the overregulation, it is necessary to ensure a smooth advance (not only at the time of detection of a certain controlled process of influence, but at the time of detection of the processes of influence that are interrelated with this controlled process of influence and usually preceded it in the previous cycles of interaction) increase of the values of the activating influences coming to the primary generators in a certain current-foreseeable situation (smoothing).

Based on the fact that the overload and overregulation have the same causes of emergence for all controlled processes of interaction (7.5.1, 7.5.2), the rules of change of the activating sequences of signal combinations coming to the primary generators are the same for all specified controlled processes of interaction. These rules reflect certain limited types of interrelation among the interrelated processes of the universe. Therefore, the processes that implement these rules shall adjust themselves to the detection of certain limited types of interrelations among the specified processes of the universe. Therefore, the implementation of these rules shall be carried out thanks to the predetermined and transferred by the hereditary mechanisms $(5.2 .1,5.2 .2)$ sets of the processes of the nervous system with such a set of the properties, the values of characteristics and the structure of interrelation (7.1) that will ensure the implementation of these rules. 


\subsection{Additional Types of Interaction of Processes of Nervous System}

\subsubsection{Types of Interaction between Preprogrammed and Self-programming Processes of Nervous System}

In the nervous system of the combined type, there can be interaction (the mutual influence, direct or indirect - 4.2.1) of the nervous system's processes that are included to certain groups (blocks) of preprogrammed and self-programmed (self-adjusting - 4.3) processes of the nervous system (4.3). When interacting with the self-programming and preprogrammed systems of the nervous system, two types of mutual influence can be implemented:

- influence on the work of preprogrammed systems by self-programming systems. This type of influence will allow to correct the sequences of signal combinations generated by the preprogrammed systems for their greater conformity to achieving TEFs of the organism taking into account the new detected processes;

- influence on the work of self-programming systems by the preprogrammed systems. This influence is necessary to implement the possibility of adjustment of certain self-programming systems to the interaction with certain types of the processes of the universe and/or their certain properties.

The adjustment of the self-programming processes of the nervous system controlled by the preprogrammed systems allows to provide the interaction that is necessary for the implementation of the strategy of achieving TEFs with the processes of the universe (5.2.2) that are critical for the implementation of this strategy. This type of influence can be implemented both by modulating the established interrelationships between the self-programming processes of the nervous system (for example, modulation of adjustment of the generators to the detection of the processes - 7.2, 7.3, 7.4) and by other ways. In case of these ways, certain sequences of signal combinations generated by selfprogramming processes of the nervous system during the detection of certain processes of the universe that correspond to certain processes or their properties or the values of characteristics detected in a certain current situation by the preprogrammed processes of the nervous system shall be more likely to influence other self-programming processes of the nervous system and, thus, lead to their self-adjustment (6.2.18) to the detection of these processes of the universe with certain properties or the values of characteristics or their certain properties with certain values of characteristics.

In case of both types of interaction of the self-programming and preprogrammed processes of the nervous system, the interrelated nervous system's processes belonging to different systems (selfprogramming and preprogrammed) shall detect (adjust themselves to the detection) the interrelated processes of the universe (5.4.1).

\subsubsection{Modulation of Adjustment of Self-adjusting (Self-programmed) Processes of Nervous System (Detectors) to Detection of Processes of the Universe during Detection of which State and Probability of Achievement of TEFs Increase}

The nervous system, perceiving the external and internal processes, determines (detects) those of them (properties, values of characteristics and cause-and-effect relations) the influence of which in the past situations was important for the organism to achieve its TEFs and, when detecting these processes in the current situation (4.1.2), generates such influences on the processes of the universe as a result of which the state or the probability of achievement of TEFs of the organism increase (4.1.3). This influence is exerted by the sequences of signal combinations generated by the generators $(4.3,7.2 .1)$. The generators adjust themselves to the detection of certain processes of the universe receiving (directly or indirectly) the influences from other nervous system's processes that detect the current situation $(7.2,7.3,7.4)$. Therefore, the increase in the efficiency and effectiveness of adjustment of the generators and, thus, the efficiency and effectiveness of achievement of TEFs of the organism is possible in case of ensuring the first-priority adjustment of the nervous system's processes that detect the current situation to the detection of such processes of the universe (such current situation - 4.1.2) during the detection of which the efficient and effective achievement of the TEFs is carried out (4.1.3). I.e., in this case, the nervous system's processes that detect the current situation can, when detecting the specified processes of the universe, also receive (directly or indirectly) the modulating signals (6.2.10) from the processes that detect 
the state of achievement of TEFs of the organism. The structure of the interrelation among the processes of the nervous system by which such a modulating influence is provided shall be predetermined, defined in previous generations and transferred through the hereditary mechanisms (5.2.1, 5.2.2).

\subsection{Derivative Properties of Interaction of Processes of Nervous System}

\subsubsection{Dependence of Generated by Process of Nervous System Signal about Accuracy of Determination of Process of the Universe on Signals of Nervous System's Processes that Detect Processes of the Universe Interrelated with the Specified Process of the Universe, Including on Relevant Generators}

The nervous system is a hierarchical process (4.1.3) that consists of the sets of interrelated hierarchical groups (5.4) of the nervous system's processes. The hierarchy and the structure of interrelation of these sets of the nervous system's processes reflect the hierarchy and the structure of interrelation of the processes of the universe and their properties (5.4.1) with which the nervous system can interact in the environment of existence of the organism (5.3.4). The processes of the nervous system adjust themselves to the detection of the signals from other nervous system's processes interrelated with them (6.2). The interaction of the nervous system's processes with the processes of the universe can be carried out both in the presence of the controlled processes of interaction (controlled by the sequences of signal combinations generated by the generators - 7.3.1), which will determine the character of change of the processes during this interaction, and as a result of the change of the specified processes of the universe as a result of their interaction with one another and/or the interaction of their subprocesses with one another or the mutual change of their properties (3.1). During the implementation of the interaction of the processes of the universe with the participation of the processes of interaction (7.3.1) controlled by sequences of signal combinations generated by the generators, the processes of influence (7.2.2) detected by the generators generating these sequences of signal combinations will also be a part of these interrelated processes of the universe. And their interrelation with other processes of the universe will be reflected in the interrelation of the generators that detect the specified processes of influence, with the corresponding nervous system's processes that detect these other processes of the universe. Hence, the processes of the nervous system, when detecting the processes of the universe, will adjust themselves (6.2) to receive signals not only from the receptors $(4.2 .2,4.3)$ or from the nervous system's processes that detect certain properties and/or subprocesses of these processes detected by them and from the detectors of other interrelated processes of the universe (and/or their properties) (5.4) that interact with it in the current situation (4.1.2) and from the detectors that detect the processes that are hierarchically higher than the specified ones (7.2.12) but also from the generators that detect the processes interrelated with these processes of influence. Therefore, the generated by the processes of the nervous system signal about the accuracy of determination of a certain process of the universe (6.2.9) depends on the total set of the specified signals (4.2.2, 6.2.3) coming to this process of the nervous system in a certain situation.

\subsubsection{Indirect Detection and Static and Dynamic Drift when Generating by Processes of Nervous System Signals about Accuracy of Process Determination}

The nervous system is a hierarchical process (4.1.3) that consists of the sets of interrelated hierarchical groups (5.4) of the nervous system's processes. The hierarchy and the structure of interrelation of these sets of the nervous system's processes reflect the hierarchy and the structure of interrelation of the processes of the universe and their properties (5.4.1) with which the nervous system can interact in the environment of existence of the organism (5.3.4).

In the nervous system with a certain level of complexity of the structure of interrelationships among the interrelated hierarchical groups of processes of the nervous system, such a level of the interrelation among the processes of the nervous system can be (therefore, and the interdependence of the generated by them signals about the accuracy of the process determination - 7.7.1, 6.2.3) at which the signal about the accuracy of determination of a certain process of the universe can be generated by a certain process of the nervous system in the absence of direct influences exerted by this process of the universe in the current situation (4.1.2) on the receptors of the nervous system only thanks to the interrelations among the 
nervous system's processes reflecting the interrelations among the processes of the universe and their properties (7.7.1). I.e. the detection of a certain process of the universe will be possible only on the basis of the signals transmitted through additional interrelationships (6.2.21) from the processes of the universe interrelated with it and/or their corresponding properties that directly or indirectly (4.2.1) interact with the specified process in certain current situations as the subprocesses of a certain process of the universe of the higher level of hierarchy (3.1). The more signals through these interrelationships influence the corresponding process of the nervous system and the greater the value of these signals are, the higher the signal about the accuracy of the process determination will be generated by this process of the nervous system (6.2). Such detection of the processes of the universe by the processes of the nervous system will hereinafter be called the indirect detection.

In the above-mentioned nervous system, thanks to the interaction of the nervous system's processes through the interrelations among them that reflect the corresponding interrelations among the processes of the universe detected by them and their properties (7.7.1), which lead to the corresponding interdependence of the signals generated by these processes of the nervous system, the generated signal about the accuracy of determination a certain process of the universe can be reduced or increased with both variable and unchangeable influences exerted by other processes of the universe, with unchangeable influences exerted by this process of the universe, in the current situation 4.1.2) on the receptors of the nervous system (respectively, the dynamic and static drift of the signal about the accuracy of the process determination).

The interrelationships among the processes of the nervous system, through which the indirect detection, the dynamic and static drift are implemented, are formed as a result of the statistical adjustment of the processes of the nervous system (6.2) during their interaction with the corresponding processes of the universe in the previous cycles of interaction according to the rules described above (6.2).

\subsubsection{Advance Indirect Detection of Processes of the Universe}

All processes of the universe are perceived by the processes of the nervous system in the form of the sequences of combinations of interrelated influences from these processes (4.2.2). These influences emerge as a result of the direct or indirect interaction between the processes of the nervous system and the processes of the universe (4.2.1). The processes of the universe that compose a certain current situation (4.1.2) can have different duration (different number of cycles of interaction of their subprocesses) and not simultaneous start and end and can be interrelated (interact with one another forming the processes of a higher level of the hierarchy - 3.1). Thus, certain interrelated processes that are implemented within a certain hierarchically higher process (are its subprocesses) can have different duration and/or shifted (one relative to other) start and end of their influence on the nervous system's receptors. Moreover, these processes in other current situations within other (hierarchically higher) processes can exert a simultaneous (3.1) influence on the receptors of the nervous system of the organism.

As specified above (7.7.1), the processes of the nervous system during the detection of the processes of the universe receive signals not only from the receptors $(4.2 .2,4.3)$ or from the nervous system's processes that detect certain properties and/or subprocesses of these detected processes, but also from the detectors of other processes interrelated with these processes of the universe and from the detectors of their corresponding (interrelated with the properties of these processes) properties (5.4) that interact with the specified processes in a certain current situation (4.1.2) as well as from the detectors that detect the processes hierarchically higher than the specified ones (7.2.12). Hence, when detecting the process of the universe that in a certain current situation is the first to exert an influence on the receptors of the nervous system, the nervous system's processes that detect this process of the universe will send the influences to the nervous system's processes that detect the processes interrelated with this process of the universe. These processes of the nervous system, in their turn, can also be interrelated (and, therefore, send the signals generated by them to it) with (or be) the nervous system's process that detects the process of the universe that exerts an influence on the nervous system's receptors some time after the first detected process of the universe.

As specified above (7.7.2), in the nervous system with a certain level of complexity, the processes of the universe can be detected indirectly - the signal about the accuracy of determination of a certain process of the universe can be generated by a certain process of the nervous system thanks to the 
interrelations among the nervous system's processes reflecting the interrelations among the processes of the universe and their properties in the absence of the direct influences exerted by this process of the universe in the current situation on the receptors of the nervous system. Therefore, when detecting a certain process/processes of the universe that, in this current situation, is the first to exert an influence on the receptors of the nervous system thanks to the interrelationships among the nervous system's processes that detect the interrelated processes of the universe (7.7.1, 7.7.2), the indirect detection is possible of interrelated, in this current situation, another process of the universe that will exert an influence on the receptors of the nervous system some time after the first detected process of the universe, before its direct interaction with the processes of the nervous system - advance indirect detection. Moreover, in the case of the indirect advance detection of a certain process of the universe, the signal from the nervous system's process that detects it can, in its turn, lead to the indirect advance detection, by the corresponding nervous system's processes interrelated with it, of the processes of the universe interrelated with this process of the universe in this current situation — cascade indirect advance detection.

Since, as specified above, in case of the advance indirect detection of processes of the universe in a certain current situation, it is possible to detect the processes of the universe interrelated with these processes that will interact with the processes of the nervous system in the future (through several cycles of interaction), the advance detection with the indirect detection of the processes of the universe, in a certain current situation, it is possible to determine the current-foreseeable (4.1.2) situation based on the detection of the processes of the universe that form the current situation.

\subsubsection{Change and/or Formation of New Interrelationships between Processes of Nervous System through Advance Indirect Detection of Processes of the Universe}

The processes of the nervous system adjust themselves to the detection of the processes of the universe by randomly forming (6.2.14) the relations with one another (in the presence of the appropriate interrelation between these processes of the nervous system - 5.4.1) and distinguishing from the sequences of signal combinations that influence them the interrelated sequence of signal combinations the influence from which statistically most frequently leads to the generation (by them) of the highest outcoming signal about the accuracy of determination of the detected process (6.2.18). In case of the advance indirect detection (7.7.3) of the processes of the universe, the simultaneous generation of signals is possible by the nervous system's processes that detect the process of the universe that is the first to influence the receptors of the nervous system, and the nervous system's processes that detect the process of the universe that is the second to influence the receptors of the nervous system. Moreover, such simultaneous generation of signals by these processes of the nervous system in a certain current situation can be implemented thanks to the advance indirect detection of the second process of the universe by the corresponding processes of the nervous system before its influence on the receptors of the nervous system. Therefore, as a result of the advance indirect detection, the nervous system's processes that detect the first process of the universe can form the relations (6.2.3) with the nervous system's processes that detect the second process of the universe, in the presence of the appropriate interrelation between these processes of the nervous system (5.4.1). I.e. the signals generated by each of these processes can be a part of the sequences of signal combinations detected by another (of these two) processes of the nervous system.

\subsubsection{Dynamic Linking of Nervous System's Processes that Detect Dynamically (Indirectly through Sequence of Interactions with Other Processes within Hierarchically Higher Processes) Related Processes of the Universe}

The processes of the universe interact according to the constant rules determined by the sets of their properties and values of characteristics (3.1). All processes of the universe are perceived by the processes of the nervous system in the form of the sequences of combinations of interrelated influences (4.2.2). These influences emerge as a result of the direct or indirect interaction between the processes of the nervous system and the processes of the universe (4.2.1). During a long-lasting (for several cycles) sequential interaction of the nervous system's processes with the one and same process of the universe, this process will influence the processes of the nervous system as a long-lasting sequence (the sequence of 
interrelated combinations) of combinations of interrelated influences. This sequential interaction, in its turn, can be carried out both in the presence of the controlled processes of interaction (controlled by the sequences of signal combinations generated by the generators - 7.3.1), that will determine the character of the change of the processes during this interaction with the specified process of the universe, and as a result of the change of this process of the universe itself as a result of the interaction of its subprocesses with one another and/or with other processes of the universe (3.1).

As specified above (6.2), the processes of the nervous system adjust themselves to the detection of the signals from other nervous system's processes interrelated with them. Besides, (7.7.1) the processes of the nervous system, when detecting the processes of the universe, receive the signals not only from the receptors $(4.2 .2,4.3)$ or from the nervous system's processes that detect certain properties and/or subprocesses of the mentioned detected processes, but also from the detectors of other processes of the universe interrelated with these detected processes and/or their properties (5.4) that interact with the specified processes in a certain current situation (4.1.2) and from the detectors that detect the processes of the universe hierarchically higher than the specified ones (7.2.12) as well as from the generators that detect the processes of influence interrelated with these processes. Hence, when a certain nervous system process detects a certain sequence of signal combinations that emerges when interacting with a certain process of the universe with which the processes of the nervous system can interact in a certain situation for a certain number of cycles of interactions and that reflects the subprocesses, the properties and the values of characteristics of this process of the universe (4.2), the specified process of the nervous system will transmit the influence on other nervous system's processes that will detect the following sequences of signal combinations that will occur during further interaction with the specified processes of the universe. As a result, the chain of sequentially interrelated processes of the nervous system will be formed that will generate signals about the accuracy of determination of the sequences of combinations of interrelated signals when interacting with a certain process of the universe. The generation of a signal by the first process of the nervous system in this sequence will eventually lead (through the chain of interactions of the nervous system's processes) in a certain current situation, during the long-lasting interaction with a certain process of the universe to generation of a signal by the last process of the nervous system included in this sequence. The described dependence between the generated by the processes of the nervous system signals about the accuracy of determination of the processes is a dynamic linking of the processes of the nervous system.

\subsubsection{Advance Detection and Dynamic Linking as Main Processes of Interaction of Processes of Nervous System during Their Interaction with Processes of the Universe}

In the universe, in which the existence of intelligent life is possible, the processes shall have such properties that will lead to the possibility of the formation of the identical (same-type) and similar localized long-lasting (much more than one cycle of interaction among the subprocesses) hierarchically higher processes $(3.1,3.2)$. Therefore, the processes of the universe, starting from a certain initial level of the hierarchy, will interact with one another mainly within the hierarchically higher identical (same-type) and similar localized long-lasting processes, some of which are the processes that are the living organisms (3.2). Taking into account the complexity (multicomponence) of these hierarchical processes and, therefore, the diversity of their properties, they (mainly) will have different duration and sequence of interactions within the hierarchically higher processes formed by them. Thus, the interaction of the nervous system's processes with the processes of the universe during the existence of a living organism in the universe with the described properties (3.1) will occur mainly with the long-lasting hierarchically higher than the initial level processes with different duration and order of interaction. Hence, the interaction of the nervous system's processes with the processes of the universe will mainly lead to the advance detection (7.7.3) and the dynamic linking (7.7.5).

\subsubsection{Multiple Interrelation of Processes of Nervous System}

The nervous system is a hierarchical process (4.1.3) that consists of the sets of interrelated hierarchical groups (5.4) of the nervous system's processes. The hierarchy and the structure of the interrelation of the groups of the processes of the nervous system reflect the hierarchy and the structure of the interrelation of 
the processes of the universe and their properties (5.4.1) with which the nervous system can interact in the environment of the organism's existence (5.3.4). Every process of the universe can interact differently with other processes of the universe and belong to different hierarchically higher processes (3.1, 6.3.2, 7.7.6). Therefore, the nervous system's process that detects a certain process of the universe can be interrelated with different nervous system's processes that detect other processes of the universe with which the specified process of the universe can interact in different current situations (4.1.2) and different hierarchically higher processes.

\subsubsection{Long-lasting Interaction of Processes of Nervous System as a Result of Indirect Detection (Indirect Generation of Signals about Accuracy of Process Determination)}

In the nervous system with a certain level of complexity, the processes of the universe can be detected indirectly - the signal about the accuracy of determination of a certain process of the universe can be generated by a certain process of the nervous system thanks to the additional (6.2.21) interrelations among the processes of the nervous system in the absence of the influences exerted by this process of the universe in the current situation on the receptors of the nervous system (7.7.2). These additional interrelationships among these processes of the nervous system reflect the interrelation of certain processes of the universe and were formed during the interaction of the nervous system with these (or similar to them - 3.1) interrelated processes of the universe in the previous cycles of interaction (7.7.2). The interaction of the nervous system's processes mainly occurs with the long-lasting hierarchically higher than the initial level processes of the universe with different duration and order of interaction (7.7.6). The interaction of the nervous system's processes with these processes of the universe leads to the sequences of interrelated sequences of combinations of interrelated signals that are detected by the processes of the nervous system (7.7.5). Such character of interaction of the nervous system's processes with the processes of the universe will mainly lead (7.7.6) to the advance detection (7.7.3) and the dynamic linking (7.7.5). Thus, in the nervous system of a certain level of complexity, at which the indirect detection of the processes is possible, the dynamically interrelated processes of the nervous system can occur, in which the whole chain (the sequence of interactions) of interaction of the processes will be realized as a result of the indirect detection of the processes of the universe.

The processes of the universe, with which the processes of the nervous system interact, interact with other processes of the universe (according to certain unchangeable rules determined by their properties and the value of characteristics - 3.1), including the nervous system's processes, forming the hierarchically higher processes of the universe. Moreover, one and the same process of the universe (or the identical [same-type] or similar process of the universe - 3.1) can interact with different (other) processes of the universe in different current situations in the composition of different hierarchically higher processes, that, in its turn, leads to multiple interrelation of the processes of the nervous systems (7.7.7). This interrelation of the processes of the universe and the corresponding multiple interrelation of the processes of the nervous system lead to the interrelation of the chains of the processes of the nervous system, through which the dynamic linking is implemented (7.7.5). Thus, in case of alternate (first with one process of the universe then with another, and so on) direct interaction with the processes of the universe, or in case of their alternate indirect detection, a transition from one chain (7.7.5) of the nervous system's processes to another is possible. Therefore, the long-lasting (greater than the duration of interaction within one chain) interaction of the interrelated nervous system's processes constituting both one and several interrelated chains is possible, both at direct and indirect detection of the processes of the universe. Moreover, the overall sequence of interactions of the nervous system's processes in the transition between the chains of the interrelated processes of the nervous system within this long-lasting indirect interaction can be unique and not implemented in the previous interactions of the nervous system's processes with the processes of the universe during the formation of the corresponding chains and their interrelations. However, individual chains and their interrelation with one another, as specified above (7.7.2), are formed during the previous interactions of the nervous system's processes with the processes of the universe. 


\subsubsection{Mixed Interaction of Processes of Nervous System as a Result of Indirect Detection and Direct Interaction with Processes of the Universe}

A living organism is a process of a certain level of the hierarchy that is constantly in a certain environment $(3.2,3.1)$ and, thus, is constantly interacting with other processes of the universe. Therefore, the receptors (at least a part of them) of the nervous system of a living organism will be constantly generating the signals as a result of a direct or indirect (4.2) interaction with other processes of the universe, including the processes of the living organism itself (4.3). Thus, in the nervous system of a certain level of complexity, in which the indirect detection of the processes of the universe is possible (7.7.2) and, thus, a long-lasting interaction of the processes of the nervous system as a result of the indirect detection (7.7.8), the nervous system's processes that receive the signals from the nervous system's processes that generate the signals as a result of the indirect detection will interact with the nervous system's processes that generate the signals about the accuracy of determination of the processes of the universe as a result of the influences from the receptors $(4.2 .2,4.3)$ or from the nervous system's processes detecting certain properties and/or subprocesses of the specified (detected indirectly) processes (5.4). I.e. a mixed interaction of the processes of the nervous system will be carried out in the described nervous system. The ratio between the generation of the signals by the processes of the nervous system as a result of the indirect detection and as a result of the direct interaction with other processes of the universe in a certain current situation will depend on the existing formed interrelationships among the nervous system's processes (6.2.3) that, in their turn, depend on the predetermined structure of the interrelationships of the processes of the nervous system, their set and properties and on previous interaction of the nervous system's processes with the processes of the universe (5.2, 7.7.1) as well as on the current situation itself $(4.1 .2,4.2)$ and on the current situations preceding it (7.7.2, 7.7.5).

\subsubsection{Increase in Current Accuracy (with Current Interaction) of Detection of Processes With Increasing Number of Cycles of Interaction of Processes of Nervous System with Processes of the Universe in Current Situation}

As specified above (4.2.2), all processes of the universe are perceived by the processes of the nervous system in the form of the sequences of combinations of interrelated influences from these processes. These influences arise as a result of the direct or indirect interaction between the processes of the nervous system and the processes of the universe (4.2.1). Different groups of subprocesses of the detected process of the universe can participate in each such interaction (some subprocesses will interact with one another or with other processes of the universe without interacting [directly or indirectly] with the processes of the nervous system), and these interactions can occur thanks to different properties $(3.1,5.4 .6)$ of these subprocesses. The nervous system of the organism has limitations in the number of the processes with which its processes can interact simultaneously (perceive influence or influence these processes - 5.3.5). The accuracy of determination of the processes of the universe by the processes of the nervous system increases with the increase in the number of the unique for these detected processes of the universe, interrelated sequences of signal combinations generated during the interaction with them $(6.2 .7,6.2 .9)$. Besides, the accuracy of the determination of a certain process of the universe by a certain process of the nervous system depends not only directly on the signals generated by the receptors or the detectors of the lower-level processes from which this process of the nervous system receives the influences, but also on the signals from other (interrelated with the specified process of the nervous system) nervous system's processes that detect the processes interrelated with the specified detected process of the universe and/or the properties of the specified process of the universe (7.7.2). In case of increase in the number of the interaction cycles (as a result of the processes that are not influenced or influenced by the sequences of signal combinations generated by the generators) of the nervous system's processes with a certain process of the universe in a certain current situation, the number of the unique (for the detected process) interrelated sequences of combinations of influences that this process or the processes of the universe interrelated with it in the current situation exert on the processes of the nervous system increases. Therefore, the possibility of increase in the accuracy of determination of the specified detected process of the universe in the current situation will increase $(6.2 .7,6.2 .9)$. 
7.7.11. Increase in Accuracy of Adjustment of Processes of Nervous System to Detection of Processes of the Universe With Increasing Number of Interaction with These Processes in Different (Similar and Dissimilar) Current Situations

All processes of the universe are perceived by the processes of the nervous system in the form of the sequences of combinations of interrelated influences from these processes (4.2.2). These influences arise as a result of the direct or indirect interaction between the processes of the nervous system and the processes of the universe (4.2.1). The processes of the nervous system adjust themselves to the detection of the processes of the universe by distinguishing from the sequences of signal combinations that influence them the interrelated sequence of signal combinations the influence from which statistically most frequently leads to the generation of the highest outcoming signal about the accuracy of determination of the detected process (6.2.18). In case of increase in the number of the cycles of interaction (as a result of the processes that are not influenced or influenced by the sequences of signal combinations generated by the generators) of the nervous system's processes with a certain process of the universe in different (similar and dissimilar) current situations, the number of the unique (for the detected process) interrelated sequences of combinations of influences that this detected process (or the processes of the universe interrelated with it in certain current situation - 7.7.2) exerts on the processes of the nervous system increases. Therefore, the possibility of a more accurate statistical adjustment of the nervous system's processes to the detection of these unique interrelated sequences of combinations of influences will increase and, thus, the accuracy of determination of the specified detected process of the universe by the processes of the nervous system will increase.

\subsubsection{Increase in Accuracy of Adjustment of Processes of Nervous System to Detection of Processes of the Universe Thanks to Interaction of Processes of Nervous System through Existing Adjusted Interrelationship in Absence of Direct Influences from Receptors on Processes of Nervous System}

The nervous system is a hierarchical process (4.1.3) that consists of the sets of interrelated hierarchical groups (5.4) of the nervous system's processes. The hierarchy and the structure of the interrelation of the groups of the processes of the nervous system reflect the hierarchy and the structure of the interrelation of the processes of the universe and their properties (5.4.1) with which the nervous system can interact in the environment of the organism's existence (5.3.4). In the nervous system with a certain level of complexity, the processes of the universe can be detected indirectly — the signal about the accuracy of determination of a certain process of the universe can be generated by a certain process of the nervous system (thanks to the additional interrelations among the processes of the nervous system) in the absence of the influences exerted by the specified process of the universe in the current situation on the receptors of the nervous system (7.7.2). Thus, the processes of the nervous system can receive the influences from the nervous system's processes, which generate the signals about the accuracy of determination of a certain process of the universe, not only in the presence of the influences caused by the interaction of this process of the universe with the receptors in a certain current situation, but also in their partial or complete absence by the indirect detection.

The processes of the nervous system adjust themselves to the detection of the processes of the universe by distinguishing from the sequences of signal combinations that influence them the interrelated sequence of signal combinations the influence from which statistically most frequently leads to generation (by them) of the highest outcoming signal about the accuracy of determination of the detected process (6.2.18). When increasing the number of the generated by the processes of the nervous system unique (for a certain detected process of the universe) interrelated sequences of combinations of influences in case of indirect detection (by this nervous system's processes) of the subprocesses of the specified process of the universe or its certain properties or the processes of the universe interrelated with it in certain current situations, the number of the unique (for the detected process) interrelated sequences of combinations of influences that these processes of the nervous system will exert on the nervous system's process that detects the specified process of the universe will increase. Therefore, the possibility of a more accurate statistical adjustment of the specified process of the nervous system to the detection of these unique interrelated sequences of combinations of influences will increase and, thus, the accuracy of determination 
of the specified detected process of the universe by this process of the nervous system will increase.

\subsubsection{Change and/or Formation of New Interrelationships between Processes of Nervous System without Their Direct Detection when Interacting with Processes of the Universe}

The processes of the nervous system can receive influences from the nervous system's processes that generate the signals about the accuracy of determination of a certain process of the universe, not only in the presence of the influences caused by the interaction of this process of the universe with the receptors in the current situation, but also in their partial or complete absence through indirect detection (7.7.2). The processes of the nervous system adjust themselves to the detection of the processes of the universe by distinguishing from the sequences of signal combinations that influence them the interrelated sequence of signal combinations the influence from which statistically most frequently leads to the generation (by them) of the highest outcoming signal about the accuracy of determination of the detected process (6.2.18). The character of interaction of the nervous system's processes with the processes of the universe will mainly lead to the advance detection and the dynamic linking (7.7.6). Moreover, in the nervous system of a certain level of complexity, the long-lasting interaction of the processes of the nervous system as a result of indirect detection (indirect generation of signals about the accuracy of determination of the processes) is possible (7.7.8). The overall sequence of interactions of the nervous system's processes in the transition between the chains of the interrelated nervous system's processes within this long-lasting indirect interaction (interaction as a result of indirect detection) can be unique and not implemented in the previous interactions of the nervous system's processes with the processes of the universe during the formation of the specified chains and their interrelations (7.7.8). Thus, taking into account the described possible uniqueness of the interrelation of the chains of the nervous system's processes during the longlasting indirect detection with advance detection (7.7.8), it is possible to form new sequences of signal combinations. Therefore, in case of their statistically significant repetition, the change of the interrelationships of the nervous system's processes (6.2.18) is possible due to such long-lasting indirect detection of the processes with their advance detection. I.e. it is possible to change and/or to form new interrelationships between the processes of the nervous system without directly detecting the specified processes of the universe. Such changes will, in their turn, lead to a change of the chains or change or formation of new interrelationships among them and, thus, to the possibility of change and/or formation of new interrelationships between the processes of the nervous system in case of long-lasting indirect detection with advance detection with the participation of these changed interrelationships.

\subsubsection{Dependence of Conformity of Changes of Interrelationships of Processes of Nervous} System to Existing Interrelations among Processes of the Universe (at Change or Formation of These Interrelations without Direct Interaction with Corresponding Processes of the Universe) on Previous Cycles of Interactions of Processes of Nervous System with Processes of the Universe

In case of long-lasting interaction of chains of the processes of the nervous system with the indirect and advance detection of the processes of the universe, change and/or formation of new interrelationships between the processes of the nervous system is possible (7.7.13). The changes in the interrelationships of the nervous system's processes resulting from such interaction can both increase the accuracy of detection of the processes of the universe or detection of new interrelationships between the interrelated processes of the universe and decrease the accuracy of their detection due to formation of interrelationships between the nervous system's processes that will not reflect the actual interrelation between the processes of the universe. Taking in consideration the above $(3.1,4.2,5.4 .1)$, the greater the conformity of the interrelationships among the nervous system's processes forming the corresponding interrelated chains of interrelated processes of the nervous system $(7.7 .5,7.7 .8)$ to the interrelationships of the detected by them interacting processes of the universe is, the more accurate the interaction of these processes of the nervous system within the long-lasting indirect detection with advance detection will reflect the interaction of the corresponding processes of the universe (the dependence among the sequences of signal combinations generated by the processes of the nervous system will correspond to the dependence among the corresponding processes of the universe, their properties and valuesof characteristics - 4.2). The conformity of the interrelationships of the processes of the nervous system to the interrelationships of the 
processes of the universe detected by them, in its turn, depends on the capabilities of the nervous system (5.3.4): the set of the processes of the nervous system predetermined and transferred through the hereditary mechanisms, the set of their properties, the values of their certain characteristics and, thus, the structure of interrelation and interrelationships (5.2.1), and the previous cycles of interaction of the processes of the nervous system with other processes of the universe (5.2.1, 6.2.18). Therefore, for a certain organism (with certain properties and capabilities of the nervous system), the greater the number of cycles of interaction of the nervous system's processes in different current situations with different processes of the universe is, the greater the probability is that the interaction of the chains of the nervous system's processes within the long-lasting indirect detection with the advance detection of the processes of the universe will more accurately reflect possible interactions between the corresponding processes of the universe. Therefore, the greater the probability is that the interrelationships of the nervous system's processes changed as a result of the interaction of the interrelated chains of the nervous system's processes within long-lasting indirect detection with advance detection will lead to a more accurate detection of the processes of the universe or to detection of new interrelationships between them.

\subsubsection{Processes of Organism - Processes of the Universe with which Nervous System's Processes that Belong to this Organism Most Frequently Interact}

The processes of the nervous system together with other processes of the organism form a hierarchically higher process - the organism $(4.1 .3,3.1)$. This leads to more frequent interaction of the nervous system's processes with other processes of the organism than with other processes of the universe that are not included to the specified organism (3.1). The main function of the nervous system's processes is to ensure the coordinated interaction of the organism's processes with one another and with other processes of the universe to ensure the most efficient and effective achievement of the TEFs of the organism $(4.1 .1,4.1 .3)$ to which this nervous system belongs. In order to implement this function, the processes of the nervous system detect the processes of the organism and other processes of the universe with which the processes of the organism interact, the properties and the values of characteristics of these processes and exert certain influences on them (4.1.3). Therefore, in every current situation, during the interaction (detection and influence) of the nervous system's processes with other processes of the universe, at least a part of the processes of the nervous system will interact with the processes of the universe that is a part of the processes of the organism to which this nervous system belongs.

As a result of the interaction of the nervous system's processes with the processes of the universe, the interrelated chains of the interrelated nervous system's processes that generate the signals about the accuracy of determination of the sequences of interrelated sequences of combinations of interrelated signals form (7.7.5, 7.7.7, 7.7.8). Therefore, during the interaction of the nervous system's processes with other processes of the universe will form the interrelated chains of the interrelated nervous system's processes in which at least a part of the chains will include the nervous system's processes that detect the processes of the organism (of different degree of the hierarchy, i.e. the processes of interaction of the organism's processes with one another) to which this nervous system belongs as well as the processes of interaction of the organism's processes with other processes of the universe.

\subsubsection{Dependence of Long-lasting Interaction of Processes of Nervous System as a Result of Indirect Detection of Processes of the Universe on Detectors of State and Probability of TEF Achievement}

In the nervous system of a certain level of complexity of interrelationships among the processes of the nervous system, the existence of the long-lasting interaction of the interrelated chains (sequences) of interrelated processes of the nervous system is possible as a result of the indirect detection of the processes of the universe (7.7.8). At least a part of these chains of the processes of the nervous system will include the nervous system's processes that detect the processes of the organism (of different degree of the hierarchy, i.e. including the processes of interaction of the organism's processes with one another) to which this nervous system belongs, and the processes of interaction of the organism's processes with other processes of the universe (7.7.15). Taking into account that the nervous system of the organism in each current situation shall ensure the efficient and effective achievement of TEFs of the organism by 
generating the influences on other processes of the universe (4.1, 4.2.3), then, accordingly, at least a part of these chains of the processes of the nervous system will include the nervous system's processes that are the generators $(7.7 .1,7.3 .1,7.2 .2)$ and the detectors of state and probability of the organism's TEF achievement (7.4.5, 7.4.2, 7.4.3, 7.4.7). As the nervous system's processes that are the detectors and the generators of the nervous system are involved in the long-lasting process of interaction of the processes of the nervous system as a result of the indirect detection of the processes of the universe, this process will depend on the state and probability of achievement of TEFs of the organism (activation [generation of signals about the accuracy of determination of the processes] of the detectors of TEF achievement 7.2.6, 7.3.3). Therefore, the course of this long-lasting process of interaction of the processes of the nervous system as a result of indirect detection of the processes of the universe will be such that the state and the probability of achievement of TEFs of the organism shall increase during the implementation of this process in the current situation (7.2.1).

\subsubsection{Dependence of Signals Generated by Processes of Nervous System during Long- lasting Direct Interaction and Interaction with Indirect Detection from Signals Generated by Them Earlier within this Interaction}

During the long-lasting interaction of the nervous system's processes (both as a result of the direct interaction with the processes of the universe and as a result of their interaction through the indirect detection of the processes of the universe - 7.7.8), repeated activation of one and same chains of interrelated processes of the nervous system is possible. Moreover, for the interaction of the nervous system's processes thanks to the indirect detection of the processes of the universe, the greater the interrelation (direct or indirect) of these chains with other chains is, the greater the probability of their mutual activation (both single and multiple). Taking into account the dynamic and static drift (7.7.2), generated by the nervous system's processes that are included to the specified chains signal about the accuracy of determination of the processes detected by them will be changing and will depend (during repeated activation of one and the same chains as a result of the direct interaction with the processes of the universe and as a result of their interaction through indirect detection of the processes of the universe), in addition to the signals generated by other processes of the nervous system, and indirectly (4.1.1) on the signals generated by these nervous system's processes themselves in the previous cycles of the current long-lasting interaction of the nervous system's processes.

\subsubsection{Long-lasting Interaction of Processes of Nervous System as a Result of Indirect Detection of Processes of the Universe as Main Process of Interaction of Processes of Nervous System in Nervous System of Certain Level of Complexity}

During the long-lasting interaction of the nervous system's processes as a result of indirect detection of the processes of the universe, the generation by the processes of the nervous system of the signals about the accuracy of determination of the processes of the universe is possible as a result of the indirect detection and as a result of the direct influence of the detected processes of the universe on the receptors of the nervous systems (7.7.9). Moreover (7.7.9), the ratio between the generation of the signals by the processes of the nervous system as a result of the indirect detection and as a result of the direct interaction with other processes of the universe in a certain current situation will depend on the existing formed interrelationships among the processes of the nervous system (3.1, 6.2.3), which, in their turn, depend on the predetermined structure of the interrelationships of the processes of the nervous system, their set and properties and on previous interaction of the nervous system's processes with the processes of the universe (5.2, 7.7.1) as well as on the current situation (4.1.2, 4.2) and on the current situations preceding it (7.7.2, 7.7.5). Therefore, in the nervous system of a certain level of complexity (predetermined - 5.2) of the structure of the interrelationships of the processes of the nervous system, their set and properties, as well as with a certain number of certain previous interactions with the processes of the universe, formation of such interrelationships among its processes is possible that will lead to such processes of long-lasting interaction of the processes of this nervous system as a result of the indirect detection of the processes of the universe at which the sequences of combinations of influences on other processes of the universe generated by the generators of this nervous system will be mainly caused and determined by the 
interaction of the processes of this nervous system within this long-lasting process and not the influence exerted by the processes of the universe on the receptors of this nervous system in the current situation. Moreover, the interaction of the nervous system's processes within the described long-lasting process of interaction of the nervous system's processes with the indirect and/or mixed detection of the processes of the universe shall lead to achievement of TEFs of the organism (7.7.16).

\subsubsection{9. "Self-awareness" and "Awareness" of Processes of the Universe as a Result of Interaction of Processes of Nervous System in Process of Their Long-lasting Interaction as a Result of Indirect Detection of Processes of the Universe}

As specified above (7.7.15), at least a part of the chains (which are included to the long-lasting process of interaction of the nervous system's processes with the indirect detection of the processes of the universe) of the interrelated processes of the nervous system will include the nervous system's processes that detect the processes of the organism (of different degree of the hierarchy, i.e. including the processes of interaction of the organism's processes with one another) to which this nervous system belongs and the processes of interaction of the organism's processes with other processes of the universe. Thus, the course of a corresponding long-lasting process to which these chains belong will depend on the nervous system's processes that detect the processes of the organism and the processes of their interaction with other processes of the universe. And the signals themselves generated by these processes of the nervous system, in their turn, will depend on the signals generated by other processes of the nervous system (7.7.1, 7.7.2), including the nervous system's processes that detect other processes of the organism and the processes of interaction of these processes of the organism with other processes of the universe and on the signals generated by these processes previously (7.7.17). Such self-dependence and dependence of the signals generated by the processes of the nervous system within the long-lasting interaction of the nervous system's processes with the indirect and mixed detection of the processes of the universe manifest themselves as "self-awareness". And the presence in this long-lasting process of the chains of nervous system's processes that detect the processes of the universe and their interaction additionally manifests itself as "awareness" of the presence of other processes of the universe and interaction with them.

The more complicated the predetermined structure of the interrelationships of the processes of the nervous system, their set and properties is, the more the number of more complicated interrelated chains of the interrelated nervous system's processes can be formed during the interaction of the nervous system's processes with the processes of the universe. Therefore, more complicated interactions of the processes of the nervous system and the interdependencies of the signals generated by them within their long-lasting interaction as a result of the indirect and/or mixed (7.7.9) detection of the processes of the universe can be formed. And, thus, the processes of "self-awareness" and "awareness" of the processes of the universe will be more complicated (of the higher level of interrelation). 


\section{Some Conclusions}

1. If the nervous system of the organism can perform the functions described in the section 7.7, then the result of the work of such a nervous system will be the presence of consciousness and selfconsciousness in the living organism to which the nervous system belongs. However, the degree of this consciousness will depend on the number, interconnectedness and complexity of dynamically interacting chains of processes of the nervous system. I.e. it will depend on the genetically determined structure of the living organism's nervous system $(5.2,5.3)$ and the amount of life experience (knowledge) gained by this living organism (7.7).

2. Recognition of objects and processes of the universe by a living organism is a dynamic process (7.7).

3. The emergence, evolution and functioning of intelligent life is possible if the universe in which this life exist has certain properties. Conversely, if there is intelligent life in a universe, then its properties must correspond to the properties necessary for the emergence, evolution, and functioning of this intelligent life. These properties are described in the section 3.1.

4. We are certain information in combination with certain algorithms of its processing that can be embodied in various forms, and not only in the biological form from which we came. 


\section{Bibliography}

Hendelman, W. (2006). Atlas of functional neuroanatomy (2nd ed.). Crc Press, Taylor \& Francis Group.

Hubel, D. H., \& Wiesel, T. N. (1962). Receptive fields, binocular interaction and functional architecture in the cat's visual cortex. The Journal of Physiology, 160(1), 106-154.

https://doi.org/10.1113/jphysiol.1962.sp006837

Kandel, E., Schwartz, J., \& Jessell, T. (Eds.). (2000). Principles of neural science (4th ed.). McGraw-Hill Companies, Incorporated.

Redgrave, P. (2007). Basal ganglia. Scholarpedia, 2(6), 1825. https://doi.org/10.4249/scholarpedia.1825

Schultz, W. (2007). Reward signals. Scholarpedia, 2(6), 2184. https://doi.org/10.4249/scholarpedia.2184

Адам, Д. (1983). Восприятие, сознание, память. Размышления биолога [Perception, consciousness, memory. Reflections of Biologist]. (Н. Соколов (Ред. перевода); Н. Алексеенко (Пер.)). Мир.

Баблоянц, А. (1990). Молекулы, динамика и жизнь. Введение в самоорганизацию материи [Molecules, dynamics and life. An introduction to Self-Organization of Matter]. (Л. Моисеева (Пер.)). Мир.

Бергман, П. Г. (1947). Введение в теорию относительности [Introduction to the theory of relativity]. (В. Гинсбург (Ред. перевода); П. Кунин \& И. Таксар (Пер.)). Государственное издателльство иностранной литературы.

Бехтерева, Н., Вартанян, И., Василевский, Н., Гоголицын, Ю., Дубровинская, Н., Зингермап, А., Иваницкий, А., Илюхина, В., Камбарова, Д., Костандов, Э., Кропотов, Ю., Медведев, В., Медведев, С., Морозов, В., Раева, С., Резникова, Т., Смирнов, В., Сороко, С., Трауготт, Н., ... Шеповальникон, А. (1988). Механизмы деятельности мозга человека [Mechanisms of human brain functioning]. (Наталья Бехтерева (Ред. перевода); Часть 1). Наука.

де Дюв, К. (1987). Путешествие в мир живой клетки [A guided tour of the living cell]. (Ю. Овчинников (Пер.)). Мир.

Дейч, С. (1970). Модели нервной системы [Models of the nervous system]. (Н. Позин \& Е. Соколов (Ред. перевода); С. Бурцева, В. Кричинский, \& Ю. Шмуклер (Пер.)). Мир.

Куффлер, С., \& Николс, Д. (1979). От нейрона к мозгу [From neuron to brain]. (Л. Магазаник (Ред. перевода); М. Каменская (Пер.)). Мир.

Михалевич, В. (Ред.). (1989). Словарь по кибернетике [A dictionary of cybernetics] (2-е изд.). Главная редакция Украинской Советской Энциклопедии имени М. П. Бажана.

Ньюкомб, Н. (2002). Развитие личности ребенка [Child development: Change over time]. (B. Белоусов (Пер.); 8-е изд.). Питер.

Палмер, Д., \& Палмер, Л. (2003). Эволюционная психология. Секреты поведения Ното sapiens [Evolutionary psychology: The ultimate origins of human behavior]. (А. Кулаков, И. Павлова, \& С. Рысев (Пер.); 2-е изд.). Прайм-Еврознак.

Паули, В. (1991). Теория относительности [Theory of relativity]. (В. Гинзбург \& В. Флоров (Ред. перевода); 3-е изд.). Наука.

Савельев, С. (2005). Происхождение мозга [The origin of brain]. Веди.

Сандригайло, Л. (1988). Анатомо-клинический атлас по невропатологии [Anatomical and clinical atlas of neuropathology] (2-е изд.). Вышэйшая школа.

Скоромец, А. А., Скоромец, А. П., \& Скоромец, Т. А. (2007). Топическая диагностика заболеваний нервной системы: Руководство для врачей [Topical diagnosis of diseases of the nervous 
system: Handbook for praktitioners]. Политехника.

Трейман, С. (2002). Этот странный квантовый мир [The odd quantum]. (В. Лебедев (Пер.)). НИЦ "Регулярная и хаотическая динамика." 